CHOLESTEROL GOAL ATTAINMENT IN HIGH RISK PATIENTS SEEN IN AN ACADEMIC INTERCITY INTERNAL MEDICINE PRACTICE. N. Afons. MD ${ }^{1}$, K. King, $\mathrm{RNC}^{1}$, B. DeLor, PharmD, BCPS ${ }^{2} ;{ }^{1}$ Wayne State University School of Medicine, Detroit, Ml; ${ }^{2}$ Pfizer Inc, Hartland, MI (Tracking ID \#73692)

BACKGROUND: A recent study demonstrated that,at best, only $38 \%$ of patients achieved NCEP-II LDL-C goals. Success rates were lowest among the patients who could benefit the most from therapy (18\% of those patients with coronary heart disease). New guidelines published in May 2001 increased the estimated number of treatment eligible patients. Our goals were (1) to evaluate the impact of the guidelines on our ability to reach LDL-goals in the high-risk patient (2) characterize the risk factor profile (including indepent risk factors) of our high-risk patients to assist us with developing quality of care improvement interventions.

METHODS: A retrospective chart review was undertaken in an inner-city faculty run internal medicine clinic between March and July 2002. Eighty-four patients deemed high-risk were included in the analysis. High-risk patients were defined as patients with established coronary heart disease (CHD), patients with non-coronary atherosclerosis, patients with diabetes mellitus (DM) and patients with 10 -year CHD risk $>20 \%$ as outlined in the ATP-III guidelines. Data collection included patient demographics, patient risk factors, lipid lowering therapy and laboratory data. Blood pressure goals were established as $<140 / 90 \mathrm{mmHg}$ for nondiabetics and $<130 / 80 \mathrm{mmHg}$ for diabetics.

RESULTS: The majority of the patients were female (69\%). The average age was $62 \pm 13.2$

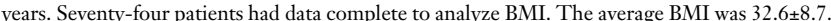
Forty-two percent (42\%) of patients had diabetes alone, 39\% had documented CHD alone, and $13 \%$ of patients had both CHD and DM. Overall LDL goal attainment was $40 \%$. Goal attainment was achieved in $42 \%$ of Diabetics and $37 \%$ of non-diabetics. The overall average percent reduction required in LDL for the population not at goal was $22.6 \%$. For diabetics not at goal the LDL reduction required to obtain goal was $22.1 \%$ and $41.9 \%$ for non-diabetics. Forty-two $(50 \%)$ patients were on lipid-lowering therapy. The average HgAlc for diabetics with a documented value was 8 . Mean triglyceride levels was 162.3. Blood pressure goal attainment was obtained in $10.9 \%$ of diabetics and $42.1 \%$ of non-diabetic patients. CONCLUSION: Achievement of LDL goal target was better than anticipated for our high risk patients. Although room to improve LDL goal attainment exists, significant additional interventional opportunities to impact CHD risk were identified through this evaluation. Obesity (BMI > 30) was present in $60 \%$ of high risk patients, smoking in $14 \%$, average $\mathrm{HgAl} \mathrm{c}$ for diabetics was 8 and blood pressure goal attainment was achieved in only $25 \%$. Targeted interventions need to focus on global risk reduction to include metabolic syndrome evaluation and intervention.

ORGANIZATIONAL CORRELATES OF QUALITY IMPROVEMENT ACTIVITIES IN VETERAN'S ADMINISTRATION HIV CLINICS. H. Anaya ${ }^{1}$, E. Yano ${ }^{2}, \mathrm{~S}$. Asch ${ }^{1} ;{ }^{1}$ VA Medical Center-West Los Angeles, Los Angeles, CA; ${ }^{2}$ Center for the Study of Healthcare Provider Behavior, Sepulveda, CA (Tracking ID \#73893)

BACKGROUND: To examine the relationship between organizational characteristics of VA HIV/AIDS clinic and quality improvement (QI) activities including clinical reminders (CR) and QI groups.

\title{
QUALITY OF CARE
}

CLINICAL INERTIA IN A FACULTY INTERNAL MEDICINE PRACTICE-FOCUS ON PROVIDER PERCEPTIONS AND NCEP III GOAL ATTAINMENT. N. Afonso ${ }^{1}$, K. King ${ }^{1}$, B. DeLor, PharmD, BCPS ${ }^{1} ;{ }^{1}$ Wayne State University School of Medicine, Detroit, MI; ${ }^{2}$ Pfizer Inc, Hartland, MI (Tracking ID \#73699)

BACKGROUND: Philips et al have described "Clinical inertia"- recognition of a problem, but failure to act. Despite evidence-based guidelines for care, providers do not initiate or intensify therapy. In the US, LDL cholesterol levels were reduced to goals consistent with the NCEP II guidelines in only $14 \%-38 \%$ of patients. The new NCEP III guidelines recommend much stricter target lipid levels. Our goal was to evaluate (1) comfort and knowledge of providers with the new treatment targets (2) provider perceptions of practice goal attainment and (3) actual LDL goal attainment with the new NCEP ATP-III guidelines.

METHODS: Four nurse practitioners and 14 physicians in an internal medicine faculty practice were surveyed to assess knowledge and perceptions of ATP III guidelines. Providers were questioned about interventions that would ensure goal attainment. To assess the translation of the ATP III guidelines into practice, 209 randomly selected charts were reviewed 9 months after the publication of the ATP III guidelines. Data collected included demographics, risk factors, laboratory information and treatment.

RESULTS: Thirteen (72\%) of practitioners, listed LDL goals that were more aggressive than ATP III guidelines for patients with 2 or more risk factors. The remaining $5(28 \%)$ practitioners listed the ATP LDL goals. All practitioners stated they were comfortable utilizing the goals established by the ATP III. Provider perceptions regarding actual goal achievement in their practice varied in response from $30 \%-70 \%$ perceived goal attainment. Evaluation of 209 patient's charts demonstrated that LDL goal attainment ranged from $90 \%$ in-patients with $0-1$ risk factors to $40 \%$ for patients with CHD or CHD Risk Equivalents. Providers ranked interventions that would help them achieve LDL goals. The majority of the practitioners listed patient education and patient assistance drug programs as the most helpful, followed by patient and provider prompts and medication compliance interventions.

CONCLUSION: Providers exceeded their perceived ability to get their low risk patients to LDL goal and overestimated their ability for patients with CHD/CHD Risk Equivalents. Targeted patient and provider interventions therefore should focus on high-risk patients. Interventions that providers feel would be most helpful are patient based solutions related to patient education and drug assistance programs. Patient and providers prompts to evaluate and intensify on-going therapy and patient compliance programs also were perceived solutions for successful goal attainment. METHODS: The source of data was the 2001 VHA Survey of HIV Programs and Practices, conducted by the Quality Enhancement Research Initiative (QUERI) for HIV/AIDS. One hundred and eighteen lead clinicians described organizational characteristics and QI activities (response rate $=86 \%$ ). Organizational characteristics included urban/rural location, geographic region, facility complexity, readiness to change, HIV caseload, HIV case management, and barriers to care. Multivariate analysis predicted quality improvement programming and use of HIV care guidelines, as well as perceived effectiveness of QI modalities including CR and QI groups, and facility and provider profiling.

RESULTS: Approximately $13 \%$ of facilities had implemented an HIV QI program; 48\% of facilities employed guidelines for HIV patient care. QI groups (3.45), and CR (3.42) had significantly higher perceived effectiveness than facility profiling (2.57) or provider profiling (2.52) (coded 1 (least effective) to 5 (most effective)). Facilities with greater numbers of HIVpositive patients were more likely to employ guidelines for HIV care. Providers at western VA facilities were more likely to think that CR and QI groups were effective than providers from the eastern, southern, and central regions. Providers from facilities with higher complexity were more likely to view CR and QI groups as effective.

CONCLUSION: Many VA HIV clinics have not yet adopted formal QI programs. As the VA and other healthcare organizations move to implement QI interventions, attention should be paid to factors affecting that implementation. Our data support two common methods, CR and QI groups, in HIV care. The association of region and facility complexity on the perceived effectiveness of both interventions should guide the VA as it moves toward systematic QI implementation.

GLOBAL QUALITY OF CARE IN THE VETERAN'S ADMINISTRATION AS COMPARED TO A NATIONAL SAMPLE. S.M. Asch ${ }^{1}$, E.A. McGlynn ${ }^{2}$, M.M. Hogan ${ }^{3}$, P.G. Shekelle ${ }^{4}$ H. Rod $^{3}$, J. Keesey ${ }^{2}$, J. Adams ${ }^{2}$, L. Rubenstein ${ }^{4}$, E.A. Kerr ${ }^{3} ;{ }^{1}$ VA, Los Angeles, CA; ${ }^{2}$ RAND, Santa Monica, CA; ${ }^{3}$ VA, Ann Arbor, Ml; ${ }^{4}$ VA Greater Los Angeles Healthcare System, Los Angeles, CA (Tracking ID \#75000)

BACKGROUND: The IOM has recommended that health delivery organizations adopt systematic approaches to quality monitoring and improvement. We compared the quality of care in the VA, which has adopted this approach, to that delivered to patients in a community sample.

METHODS: We collected medical records for 597 randomly selected VA patients from 26 facilities in 2 VISNs, oversampling for COPD, hypertension, and diabetes (response rate $=96 \%$ ). The comparison sample was 992 patients? records drawn from 7148 respondents to an ongoing random-digit dial survey of persons from 12 communities representing metropolitan statistical areas with $>200,000$ population (response rate $=48 \%$ ). National sample nonrespondents were more likely to be younger and had fewer conditions than respondents. All 
sampled patients were male, over age 35 , and had at least 2 health care visits in each of 2 years. The national subsample was weighted to match the VA sample proportions on age and the target conditions. Trained nurses abstracted all medical records using a modified version of QATools, a chart-based quality measurement instrument that uses 347 indicators for 26 conditions. We estimated the proportion of essential processes delivered to eligible patients for each target condition, as well as aggregate scores for acute, chronic, and preventive care. RESULTS: VA patients received higher overall ( $66 \%$ vs. $50 \%$ ), chronic disease ( $72 \%$ vs. $58 \%$ ), preventive ( $63 \%$ vs. $41 \%)$, diabetes ( $67 \%$ vs. $52 \%)$, and hypertension quality scores $(80 \%$ vs. $64 \%)$. Scores for COPD (69\% vs. $60 \%)$ and acute care (53\% vs. $56 \%)$ were not significantly different.

CONCLUSION: VA patients in 2 VISNs received higher overall quality of care than did national community comparisons. The VA has invested heavily in systems for assuring quality and in a comprehensive electronic medical record, possibly explaining this difference. The VA advantage, however, did not persist for acute or COPD care. Differential documentation practices, geographical variation or residual uncorrected sampling techniques could also account for some of the differences between the VA and community samples. While both samples showed significant room for improvement, our results suggest the VA can serve as a model for other systems wishing to improve their care.

PREDICTING MORTALITY IN PATIENTS WITH COMMUNITY-ACQUIRED PNEUMONIA: A COMPARISON OF 3 PROGNOSTIC MODELS. D. Aujesky ${ }^{1}$, T.E. Auble ${ }^{1}$, D.M. Yealy ${ }^{1}$ D.S. Obrosky ${ }^{1}$, R.A. Stone ${ }^{1}$, M.J. Fine ${ }^{1}$; ${ }^{1}$ University of Pittsburgh, Pittsburgh, PA (Tracking ID \#73586)

BACKGROUND: The Pneumonia Severity Index (PSI), the British Thoracic Society (BTS) rule, and its modified version (mBTS) are well validated prognostic models for communityacquired pneumonia (CAP), yet no studies have compared the predictive performance of these models. We prospectively evaluated the accuracy of these models in predicting overall 30-day mortality and in identifying patients (pts) with CAP at low-risk of short-term mortality. METHODS: We studied 3,171 adult in- and out-pts, meeting standard clinical and radiographic criteria of CAP, from 32 hospital emergency departments in Pennsylvania and Connecticut (1/15/01-8/31/01). All pts had complete follow-up data for 30-day mortality. We dichotomized pts as low vs. high risk in all 3 models using the following thresholds: PSI risk classes I-III vs. IV/V, BTS and mBTS scores $<2$ vs. $\geq 2$. Based on these cut-points, we calculated the area under the receiver operating characteristic curve (ROC), sensitivity, specificity, negative predictive value (NPV), and negative likelihood ratio (nLHR) for each rule. RESULTS: See table.

CONCLUSION: Overall, the PSI is more accurate than the two BTS rules in predicting 30day mortality for CAP. PSI is also more accurate in identifying low-risk pts, and as such, may be more useful in safely identifying the most appropriate pts for out-pt treatment.

$\begin{array}{lccc} & \text { PSI (95\% Cl) } & \text { BTS (95\% Cl) } & \text { mBTS (95\% Cl) } \\ \text { Low risk pts (\%) } & 68(66-69) & 83(82-85) & 81(80-83) \\ \text { Mortality in low-risk pts (\%) } & 1.5(1-2) & 3.3(2.6-4) & 2.9(2.3-3.7) \\ \text { Mortality in high-risk pts (\%) } & 11.1(9-13.2) & 11.1(8.6-14.1) & 11.7(9.2-14.6) \\ \text { Area under ROC } & 0.74(0.71-0.8) & 0.63(0.58-0.67) & 0.65(0.61-0.69) \\ \text { Sensitivity (\%) } & 78.6(71.1-85) & 40(32-48.5) & 47.6(39.2-56.1) \\ \text { Specificity (\%) } & 69.8(68.1-71.4) & 84.4(83.1-85.7) & 82.8(81.4-84.1) \\ \text { NPV (\%) } & 98.6(98-99) & 96.7(96-97.4) & 97.1(96.3-97.7) \\ \text { nLHR } & 0.31(0.22-0.42) & 0.71(0.62-0.81) & 0.63(0.54-0.74)\end{array}$

TRENDS IN POST-DISCHARGE MORTALITY AND READMISSIONS: HAS LENGTH OF STAY DECLINED TOO FAR? D.W. Baker ${ }^{1}$, D. Einstadter ${ }^{2}$, S.S. Husak ${ }^{2}$, R.D. Cebul ${ }^{2}$; ${ }^{1}$ Northwestern University, Chicago, IL; ${ }^{2}$ MetroHealth Medical Center, Cleveland, $\mathrm{OH}$ (Tracking ID \#75484)

BACKGROUND: Length of hospital stay (LOS) declined dramatically over the last decade, but it is not known whether this had adverse consequences. This study sought to determine 1) trends in 30-day post-discharge mortality and all-cause readmission rates from 1991-97 and 2) whether patients whose LOS was much shorter than expected had higher 30-day postdischarge mortality and readmission rates compared to patients whose observed LOS was approximately as expected.

METHODS: We conducted a longitudinal cohort study of Medicare patients discharged alive from all hospitals in Northeast Ohio after a first admission for acute myocardial infarction (AMI; $\mathrm{N}=8612$ ), congestive heart failure ( $\mathrm{CHF} ; \mathrm{N}=22,203$ ), gastrointestinal hemorrhage (GIH; $\mathrm{N}=8178$ ), chronic obstructive pulmonary disease (COPD; $\mathrm{N}=10,566)$, pneumonia ( $N=21,113)$, or stroke $(12,773)$. Medicare Provider Analysis and Review (MEDPAR) files were used to track death within 30 days of discharge and all-cause readmission within 30 days of discharge. Trends in 30-day post-discharge mortality and readmission rates were determined using multivariate logistic regression models with adjustment for admission severity of illness. Expected LOS was calculated using previously published risk-adjustment models, and the ratio of Observed to Expected (O/E) LOS calculated. The adjusted relative risk of death and readmission for patients in the lowest decile of $\mathrm{O} / \mathrm{E}$ LOS was then determined, using patients in deciles 5 and 6 as the reference category. All analyses were stratified by whether a do-notresuscitate (DNR) order was written within 2 days of admission (early), later during hospitalization, or not at all.

RESULTS: Between 1991-97, the adjusted relative risk (ARR) of post-discharge mortality rose significantly for patients with AMI (ARR 1.65, 95\% CI 1.24-2.19) and stroke (ARR 1.59, $95 \%$ CI $1.27-1.97$ ). There was no significant change in post-discharge mortality for CHF, GI hemorrhage, COPD, and pneumonia. Trends in post-discharge mortality differed according to whether a DNR order was written. For patients who did not have a DNR order written, the risk of death during the 30 days after discharge remained stable from 1991-97, with the exception of AMI (ARR 1.47 (95\% CI 1.01-2.13). In contrast, the risk adjusted post-discharge mortality rate for patients with early DNR orders increased for all diagnoses. Markedly shorter than expected LOS was associated with higher than expected risk-adjusted mortality for patients with early DNR orders but not for others (no DNR and late DNR). Risk-adjusted readmission rates remained stable from 1991-97, except for a $15 \%$ (95\% CI 3-30\%) increase for patients with CHF. Markedly shorter than expected length of stay was not associated with higher readmission rates.

CONCLUSION: The dramatic decline in length of stay from 1991-97 was not associated with worse post-discharge outcomes for patients without DNR orders. However, postdischarge mortality increased among patients with early DNR orders, and some of this trend may be due to patients being discharged more rapidly than previously.

\section{“I SAW THIS AD LAST NIGHT. ..": DIRECT-TO-CONSUMER ADVERTISING AND THE} INCIDENCE OF PATIENT REQUESTS FOR MEDICATIONS. K.D. Baum ${ }^{1}$, J.S. Hallberg ${ }^{2}$ D. Power ${ }^{2} ;{ }^{1}$ University of Minnesota, St. Paul, MN; ${ }^{2}$ University of Minnesota, Minneapolis, MN (Tracking ID \#76442)

BACKGROUND: Despite the ever-increasing resources targeted by pharmaceutical companies for direct-to-consumer (DTC) advertising of medications, little is known about the frequency of requests from primary care patients for these medications or where patients get their information. This study examines the incidence of requests for medications due to DTC advertising as well as exploring how patients learned about the medication in two primary care clinics.

METHODS: All patients checking in for appointments at two primary care clinics during two consecutive weeks were given written, voluntary, and anonymous surveys while in the waiting room. Patients were asked if they planned on discussing a medication during their appointment that they had learned of outside a doctor's office during their appointment, where they had learned of the drugs, how strongly they felt they needed the medication, and demographic information. Trained personnel were available on-site to answer questions.

RESULTS: Of the 885 surveys returned, $99(11.2 \%)$ planned to ask their health care professional during that visit about a specific medication they had learned of outside a doctor's office. $41 \%$ of patients were very sure or sure that they wanted a prescription for that medication that day. $33 \%$ of patients planning to ask about a drug made the appointment specifically for this reason. Patients learned about the drug in many ways (patients could mark $>1$ response): Television $(55 \%)$, magazines $(21 \%)$, friends $(21 \%)$, family $(15 \%)$, newspapers $(10 \%)$, radio $(10 \%)$, internet $(9 \%)$, and billboards/signs $(3 \%)$.

CONCLUSION: Patients commonly want to discuss DTC medications with their primary care physicians. Patients learn of DTC medications from multiple venues and are fairly certain that they want that specific drug. DTC advertising is having a clear impact on the number of appointments made by patients as well as the daily discussions held in clinics. As other countries continue to consider relaxing their laws on DTC advertising, further research is called for assessing the impact that these ads have upon burdened healthcare systems and practitioners.

PROVIDER ATTITUDES TOWARDS PAIN MANAGEMENT IN METHADONE MAINTENANCE PATIENTS. K.M. Berg ${ }^{1}$, A.E. Blank ${ }^{1}$, P.A. Selwyn ${ }^{1}$, M.N. Gourevitch ${ }^{1}$ J.H. Arnsten ${ }^{1} ;{ }^{1}$ Montefiore Medical Center, New York, NY (Tracking ID \#76134)

BACKGROUND: Methadone maintenance treatment (MMT) is the most widely used and efficacious form of opioid agonist therapy for opiate dependence. Previous studies have shown that MMT patients have both a high prevalence of chronic pain $(37-60 \%)$ and lower pain thresholds than matched controls. Undertreatment of pain, which occurs in all populations, is more frequent in patients with a substance abuse history, yet little is known about providerrelated barriers towards pain management in MMT patients.

METHODS: To explore this issue, we surveyed providers in a large substance abuse treatment program in the Bronx, New York. Program providers (physicians and physician assistants) deliver both primary care and substance abuse treatment in the same clinical setting, and the predominant form of substance abuse treatment is MMT. The survey assessed providers' perceived level of competency with and barriers to pain management.

RESULTS: 28 providers (100\%) completed the survey; the majority (64\%) were female and the mean age was 40 . Most $(82 \%)$ identified as generalists, and spent the majority of their time (median 30 hours/week) in direct patient care. On a 4-point scale, $100 \%$ of physicians and $82 \%$ of physician assistants rated the pain management training they received in school as "poor" or "very poor", and $78 \%$ felt that they lacked competence in pain management. On a 5 -point scale, $61 \%$ felt that they lacked knowledge regarding pain management, and $68 \%$ felt that they needed more access to pain specialists. The majority of providers also were "somewhat", "quite" or "very" concerned about abuse of prescribed medications (79\%) and about diversion of medications for profit $(68 \%)$. However, only $11 \%$ were "very" concerned about abuse, and only $7 \%$ were "very" concerned about diversion. $50 \%$ of providers were "somewhat" or "very" reluctant to prescribe opioids to drug users (but only 7\% were "very" reluctant), and over half (57\%) distrusted patients' reports of pain (but only $4 \%$ were "very" distrustful).

CONCLUSION: Among primary care providers working in a substance abuse treatment program, concerns regarding ability to manage pain effectively include lack of knowledge, lack of access to pain specialists, opioid abuse and diversion, distrust of patients' self-reports of pain, and reluctance to prescribe opioids to methadone patients. Only a small proportion of clinicians expressed very strong beliefs that might interfere with their ability to manage their patients' pain syndromes effectively. Nonetheless, our results highlight the need for improved provider education to ensure safe and effective pain management for all patients, including current and former drug users.

DIABETES CARE IN THE UNITED STATES: DO DIFFERENCES BY GENDER EXIST? D. Bonds ${ }^{1}$, S. Palla ${ }^{1}$, D. Goff ${ }^{1}$; ${ }^{1}$ Wake Forest University, Winston-Salem, NC (Tracking ID \#76096)

BACKGROUND: Research has shown variation in medical care by patient gender. Few studies have examined care provided to patients with diabetes. Our aim was to determine if variation by gender exists in outpatient services provided to patients with diabetes. 
METHODS: We used data from the National Ambulatory Medical Care Survey and the National Hospital Ambulatory Medical Care Survey. These are annual, nationwide, crosssectional surveys of patient visits to outpatient physicians and hospital clinics. To assure adequate sample size, we combined the 1999 and 2000 surveys. Analyses were limited to adults, 18 years of age. Seven clinical services were examined: measurement of blood pressure; ordering a urinalysis, cholesterol or other blood test (proxy for Hba1c); counseling regarding diet, exercise or tobacco use. Multivariable analysis was performed with adjustment for race, insurance, type of visit and co-morbidity.

RESULTS: 4,298 individuals with a diagnosis of diabetes (2,417 women) were included Differences in race (women: $53 \%$ white, $27 \%$ black, $20 \%$ other; men: $59 \%$ white, $21 \%$ black, $19 \%$ other, $\mathrm{p}<0.001$ ) and insurance status (women: $25 \%$ private, $59 \%$ public, $16 \%$ other; men: $34 \%$ private, $49 \%$ public, $17 \%$ other, $\mathrm{p}<0.001$ ) were found. There were minimal differences between genders in the receipt of clinical services with fewer men receiving a urinalysis (OR 0.83 , CI 0.69-0.99) but more receiving counseling on tobacco use (OR 1.53, CI 1.06-2.21) Overall, the percent of patients with diabetes receiving recommended clinical services was low. CONCLUSION: Little variation by gender in receipt of clinical services for patients with diabetes was found. The number receiving recommended clinical services was low for both genders. Future work improving care to all people with diabetes is needed.

Table 1: Comparison of Clinical Services

$\begin{array}{lccc} & \text { Women } & \text { Men } & \text { OR Men/Women } \\ \text { Blood Pressure } & 72 \%(1738 / 2417) & 71 \%(1340 / 1881) & 0.96(0.84-1.10) \\ \text { Urinalysis } & 15 \%(351 / 2417) & 12 \%(234 / 1881) & 0.83(0.69-0.99) \\ \text { Cholesterol } & 8 \%(205 / 2417) & 10 \%(186 / 1881) & 1.17(0.95-1.44) \\ \text { Other Blood Test } & 42 \%(1013 / 2417) & 42 \%(792 / 1881) & 1.01(0.89-1.14) \\ \text { Diet Counseling } & 36 \%(871 / 2417) & 37 \%(705 / 1881) & 1.03(0.91-1.17) \\ \text { Exercise Counseling } & 18 \%(426 / 2417) & 20 \%(371 / 1881) & 1.10(0.94-1.29) \\ \text { Tobacco Counseling } & 2 \%(55 / 2417) & 4 \%(66 / 1881) & 1.53(1.06-2.21)\end{array}$

PAIN MANAGEMENT: MEDICAL CARE FACTORS AFFECTING PAIN RELIEF. P.A. Bovier ${ }^{1}$, A. Charvet ${ }^{1}$, A. Cleopas ${ }^{1}$, N. Vogt ${ }^{1}$, T.V. Perneger ${ }^{1} ;{ }^{1}$ Geneva University Hospitals, Geneva 14, Geneva, Switzerland (Tracking ID \#76064)

BACKGROUND: Pain is a frequent complaint among hospitalized patients, and pain relief an important aspect of quality of care. However pain relief is not always achieved during hospital stays, despite the availibility of potent drugs, assessment tools, and appropriate guidelines. In this study we looked at factors associated with adequate pain relief among patients hospitalized in a teaching hospital.

METHODS: We surveyed all patients over 18 years discharged alive in March 2001 from Geneva University Hospitals. Patients autoadministered the Picker questionnaire, with additional questions developed for the pain section. These questions addressed information given by medical staff about pain and its management, regular pain assessment, use of a pain assessment tool, modification of treatment when necessary, and overall pain relief during hospital stay. All patients who had ever been in any pain during their stay were included in the analyses. Cross-tabulation, chi-square tests and logistic regression models were used to identify patient characteristics and medical factors associated with pain relief.

RESULTS: After two reminders, 1518 (70\%) of 2156 eligible patients returned the survey. A majority $(\mathrm{N}=1050,69 \%)$ had experienced pain during their stay, of whom only $66 \%(\mathrm{~N}=697)$ reported complete pain relief. Complete pain relief was more frequently reported by women, patients over 24 and under 84 years, patients with only 1 hospital stay in past 6 months, and patients with better general or mental health. The following medical care factors were associated with pain relief: systematic information about pain relief and its management, regular pain assessment, modification of pain treatment, shorter waiting time before pain killer administration, good availibility of medical doctors or nurses. Patients who stayed in gynecology-obstetrics and surgery wards were more likely to report complete pain relief Adjusted for sex, age, general health and medical departments, good availability of doctors (OR: gradient from 1.0 (poor, reference category) to 11.9 (excellent), 95\% CI: 3.7 to 37.7 ), systematic information about pain relief (OR: $2.3,95 \%$ CI: 1.5 to 3.4), regular pain assessment (OR: $1.9,95 \%$ CI: 1.3 to 2.8 ), pain treatment modification when needed (OR: $5.7,95 \%$ CI: 3.2 to 10.1 ) and waiting less than 10 minutes (OR: $5.1,95 \%$ CI: 2.8 to 9.4 ) were associated with pain relief

CONCLUSION: Only two-thirds of hospitalized patients reported appropriate pain relief during hospital stay. Several medical care factors were associated with successful pain control. These findings can guide interventions to improve quality of care.

DETERMINANTS OF MENTAL HEALTH AMONG PRIMARY CARE PHYSICIANS P.A. Bovier ${ }^{1}$, C. Goehring ${ }^{2}$, M. Bouvier-Gallacchi ${ }^{3}$, B. Kuenzi ${ }^{4} ;{ }^{1}$ Geneva University Hospitals, Geneva 14, Geneva, Switzerland; ${ }^{2}$ General Internist, Biasca, Ticino, Switzerland; ${ }^{3}$ General Internist, Melide, Ticino, Switzerland; ${ }^{4}$ Generalist, Guemligen, Bern Switzerland (Tracking ID \#76550)

BACKGROUND: In recent years, physicians have been subject to increasing economic constraints, decreasing work-related satisfaction, and increasing the risk of burnout. In this context, it is important to determine how these factors affect their health.

METHODS: We surveyed 2'756 randomly selected Swiss primary care physicians to explore the relationships between mental health, burn-out, and work-related satisfaction. Previously validated instruments were used to measure mental health (SF-12) and burnout (MBI: Maslach Burnout Inventory). Work-related satisfaction was measured with a locally developed and validated 17 -items instrument. Answers to SF-12 items were used to compute summary scores of mental and physical health. The three summary measures of the MBI (emotional exhaustion, depersonalization, personal accomplishment) and the five summary measures of the workrelated satisfaction scale (patient care, work-related burden, income-prestige, personal rewards and professional relations with colleagues) were reconstructed according to the algorythms of the original works. Linear regression models, with mental health as dependant variable, were used to study associations and interactions between these scales. All continuous variables were standardized to mean 0 and standard deviation (SD) 1 to facilitate comparisons.

RESULTS: After two reminders, 1784 physicians (65\%) responded to the survey. The majority were men $(1491,66 \%)$ and in solo practice $(1214,68 \%)$. The mean age was 51 years (SD 8.7 ; quartiles: $45-51-57)$. Half $(848,47 \%)$ were board-certified generalists, $25 \%$ (446) general internists, $9 \%$ (168) pediatricians, $9 \%$ (151) internal medicine specialists, and $10 \%$ (171) non specialist practitionners. In bivariate analyses, mental health was positively associated with personal accomplishment and all dimensions of work-related satisfaction (Pearson coefficient: $0.31,0.28,0.41,0.33,0.41,0.22$ respectively) and negatively with emotional exhaustion, depersonalization, and total hours of work per week $(-0.64,-0.34,-0.08$ respectively). In multivariate analyses, mental health was lower for women $(-0.10 \mathrm{SD})$, and for physicians over 44 and under 56 years $(-0.11 \mathrm{SD})$. Mental health was also higher among respondents with lower emotional exhaustion $(-0.50 \mathrm{SD})$, higher personal accomplishment scores (0.18 SD), higher work-related satisfaction with work-related burden (0.07 SD), income-prestige $(0.11 \mathrm{SD})$, and with personal rewards $(0.09 \mathrm{SD})$. Interaction terms between emotional exhaustion and satisfaction with income-prestige and between personal accomplishment and satisfaction with work-related burden were significant, suggesting than higher work satisfaction can buffer the regative effect of burnout on mental health. Altogether these factors explained $49 \%$ of the total variance of mental health.

CONCLUSION: Burnout and work satisfaction are important determinant of mental health of Swiss primary care physicians. Maintaining their work-related satisfaction toward incomeprestige and professional burden may prevent them from burnout.

SURVEILLANCE OF OUTPATIENT ADVERSE DRUG EVENTS - A PILOT STUDY USING THE NATIONAL ELECTRONIC INJURY SURVEILLANCE SYSTEM. D. Budnitz ${ }^{1}$ D. Pollock ${ }^{1}$, L. Annest ${ }^{1}$, S. Elbert ${ }^{2}$, A. McDonald ${ }^{2}$; ${ }^{1}$ Centers for Disease Control and Prevention (CDC), Atlanta, GA; ${ }^{2}$ Consumer Product Safety Commission, Washington, DC (Tracking ID \#73730)

BACKGROUND: Previous studies of outpatient adverse drug events (ADEs) have been restricted to patient surveys or single institutions. The limitations of such studies prompted the Institute of Medicine and the Congressional General Accounting Office to recommend innovative approaches for ADE surveillance. We implemented a pilot study to determine the feasibility of using the National Electronic Injury Surveillance System-All Injury Program (NEISS-AIP) for active surveillance of acute, outpatient ADEs.

METHODS: NEISS-AIP uses medical chart review for timely reporting of injuries treated in a national probability sample of 64 U.S. hospital emergency departments (EDs). For this study, chart reviewers from a stratified convenience sample of 9 NEISS-AIP hospitals were trained to recognize and electronically report physician-diagnosed ADEs for patients treated between July 17 and September 30, 2002.

RESULTS: Of the 546 patients ( $2.2 \%$ of all injured patients) presenting for acute care of ADEs, $8.6 \%$ were hospitalized for further treatment compared with $4.3 \%$ for all other injured patients $(\mathrm{p}<0.0001)$. The most common reasons for ADE-related ED visits were rash/pruritis (20\%), hypoglycemia (12\%), and nausea/vomiting/abdominal pain $(10 \%)$. The most common medication classes involved were anti-infective agents $(18 \%)$, diabetic agents $(12 \%)$, and cardiovascular agents (9\%). Most ADEs were adverse drug reactions (56\%). Other ADEs resulted from unintentional overdoses of medications that require close monitoring $(19 \%)$ such as insulin, anticoagulants or anticonvulsants, inappropriate use of medications by the patient or caregiver $(14 \%)$, and unintentional ingestions of medications by children $(10 \%)$. Data collection was $\geq 90 \%$ complete for core variables including drug name, route of administration, event description, and ED intervention. Most ADEs (77\%) were reported within seven days of the ED visit.

CONCLUSION: The results of this multi-institution study demonstrate that using NEISSAIP for detailed, timely surveillance of acute ADEs is feasible. ADE surveillance should be expanded to all NEISS-AIP hospitals to provide national estimates to guide development of prevention strategies.

MEDICATION ERRORS IN A COUNTY TEACHING HOSPITAL. D. Buscemi ${ }^{1}$, A. Kumar ${ }^{1}$, K. Nugent ${ }^{1}$, R. Horne ${ }^{2}$; ${ }^{1}$ Texas Tech University Health Sciences Center, Lubbock, TX; ${ }^{2}$ University Medical Center, Lubbock, TX (Tracking ID \#73734)

BACKGROUND: The 1999 Institute of Medicine reported that 44,000-98,000 Americans die each year from medical errors. Many of these represent medication errors. Knowing the number and type of errors that occur in hospitals would allow the development of interventions to limit the number of medication errors. A prospective cohort study done in 1995 detected incidents by stimulated self-report by nurses and pharmacists and by daily review of all charts by nurse investigators. (JAMA. 1995; 274:29-34). This study provided a comprehensive analysis of all steps required for drug delivery to hospitalized patients. However, these methods are not practical for most institutions.

METHODS: We wanted to determine frequency of medication errors throughout our hospital using standard nursing and pharmacy protocols. All units in this 354 -bed teaching hospital were studied. When nursing or pharmacy personnel detected a medication error, a standard form was completed and sent to the pharmacy. Using criteria described in the JAMA report, the pharmacist classified the error. The information was entered into a standard database for analysis.

RESULTS: Routine monitoring by pharmacists and nurses identified 1278 total errors ( 734 potential errors and 544 actual errors) during the first nine months of 2002 . This resulted in an error rate of 8.7 per 100 admissions or 0.9 per 1000 doses. These included 985 ordering errors (77\%), 64 transcribing errors (5\%), 68 preparation errors (5\%), 139 administering errors $(11 \%)$, and 22 other errors(2\%). The top three categories were wrong dose $(607,47 \%)$, prescribing error $(190,15 \%)$, and missed dose $(176,14 \%)$. The most frequent drug categories associated with errors were antibiotics $(307,24 \%)$, cardiovascular medications $(148,12 \%)$, and electrolytes $(139,11 \%)$. The inpatient services with the largest number of errors were internal 
medicine (471), surgery (317), and pediatrics (172). Thirty two percent of the errors occurred in intensive care units.

CONCLUSION: This study demonstrates that physicians make frequent ordering errors and that either the pharmacist or the nurse intercepts many of these. This study also demonstrates that routine monitoring can identify services and drug types associated with a higher frequency of errors. These results provide information which could direct institutional changes to promote drug safety. Potential approaches include physician education/feedback and additional safety checks with some classes of medications (eg., antibiotics in our hospital).

UNNECESSARY HOSPITAL DAYS ON GENERAL MEDICINE WARDS. M. Carey ${ }^{1}$ H. Sheth ${ }^{1}$, R.S. Braithwaite ${ }^{1}$; ${ }^{1}$ University of Pittsburgh, Pittsburgh, PA (Tracking ID \#76384)

BACKGROUND: Unnecessary hospitalizations result in needless exposures to iatrogenic morbidity and higher medical costs. Previous reports, conducted when length of stays were longer, suggested that a substantial proportion of hospital days were unnecessary for medical care. Our purpose was to estimate the current proportion of unnecessary hospital days on general medicine wards.

METHODS: We prospectively gathered data for a continuous 5 week period on all general medicine inpatients cared for by medical housestaff at a university-affiliated urban tertiary care hospital. Approximately half of the patients were cared for by hospitalists and the other half by private attendings. Each day, every senior resident was asked to categorize patients into the following mutually exclusive categories: (1) no delay awaiting medical care or discharge arrangements (2) delay awaiting medical care (3) delay awaiting discharge arrangements. Delays were defined as processes that unnecessarily prolonged hospitalization, and were measured in daily intervals. Reasons for delays were categorized using a mutually exclusive taxonomy based on previous published reports.

RESULTS: During the period of study, 2822 patient days were eligible for evaluation, of which data was obtained for $2753(97.6 \%)$. A total of $368(13.4 \%)$ hospital days were judged unnecessary for medical care, of which $230(62.5 \%)$ were due to delays awaiting discharge arrangements, and $138(37.5 \%)$ were due to delays awaiting medical care. Unnecessary hospital days were $39 \%$ more likely on weekend days (16.8\% of all hospital days) than weekdays (12.1\% of hospital days), a statistically significant difference $(\mathrm{p}=.006)$. Most unnecessary discharge delays $(84.3 \%)$ involved difficulty finding skilled nursing facility (SNF) beds, and most SNF discharges were complicated by additional factors including restrictive or uncertain patient preference $(27.3 \%)$, inadequate or delayed insurance reimbursement $(16.5 \%)$, and infections with resistant organisms $(10.3 \%)$. Of the 138 unnecessary days due to delays in medical care, $49.3 \%, 19.2 \%$, and $13.0 \%$ were due to procedures, tests, and consults, respectively, and most medical delays overall involved surgeons (23.2\%), radiologists (20.3\%), cardiologists (17.4\%), or gastroenterologists (13.0\%).

CONCLUSION: Delays in medical care and discharge arrangements may expose a significant number of patients to unnecessary iatrogenic harm despite current emphasis on decreasing length of stay. A disproportionate amount of unnecessary days occur on weekends.

THE RELATIONSHIP OF PATIENTS' EVALUATION OF PHYSICIAN'S COMMUNICATION SKILLS WITH QUALITY OF CARE MEASUREMENT BY CHART, INTERVIEW, AND CLAIMS DATA. J.T. Chang ${ }^{1}$, P.G. Shekelle ${ }^{2}$, C.H. MacLean ${ }^{2}$, D. Saliba ${ }^{2}$, C.P. Roth ${ }^{2}$, D.H. Solomon ${ }^{2}$, C. Kamberg ${ }^{3}$, R.T. Young ${ }^{1}$, N.S. Wenger ${ }^{1}$; ${ }^{1}$ University of California, Los Angeles, Los Angeles, CA; ${ }^{2}$ RAND, Santa Monica, CA; ${ }^{3}$ RAND, Arlington, VA (Tracking ID \#76286)

BACKGROUND: Increasing numbers of administrative data-based, and, to a lesser degree, chart-based measurements are used to estimate quality of care. However, patients tend to be unimpressed by these measures, while surveys demonstrate that patients highly value providers' communication skills. Yet, little is known about the relationship of quality of care and providers' communication skills. Using a set of process of care quality assessment indicators developed by the Assessing Care of Vulnerable Elders (ACOVE) project, we examined the relationship between patient reports of their providers' communication abilities and quality of care measured by patient interview, medical record abstraction and analysis of administrative data.

METHODS: ACOVE developed a set of quality indicators (QIs) for vulnerable communitybased older adults (at increased risk for functional decline and death). Patient ratings of communication with his/her health provider were measured by interview using items from the Consumer Assessment of Health Plans (CAHPS) Medicare Managed Care questionnaire. The ACOVE assessment was applied to a random sample of 420 vulnerable older adults selected from two senior managed care plans; data for a 13-month period were available from all sources for 225 patients. Quality of care assessments from medical record review (using 154 QIs), interview (using $93 \mathrm{QIs}$ ), and claims data (using $36 \mathrm{QIs}$ ) were compared to patients' communication ratings.

RESULTS: Better communication skills were associated with better quality of care as assessed by interview $(\mathrm{p}=0.009)$, but were weakly associated with quality of care as assessed by medical record review $(p=0.07)$ and not associated with quality based on claims data $(p=0.7)$. In a multivariate model including gender, race, and health status, communication skill $(\beta=0.20$, $\mathrm{p}=0.002)$ was related to quality of care assessed by interview.

CONCLUSION: Quality of care as measured by administrative data and medical record review misses aspects of care related to physician communication skills. The spectrum of care measured by particular quality measures should be considered in interpretation of quality scores and in selection of measurement tools for application to patients.

PRIMARY CARE PATIENTS' INVOLVEMENT IN DECISION MAKING IS ASSOCIATED WITH IMPROVEMENT IN DEPRESSION. S.L. Clever $^{1}$, D.E. Ford ${ }^{1}$, L.V. Rubenstein ${ }^{2}$, K.M. Rost ${ }^{3}$, L. Meredith ${ }^{2}$, C.D. Sherbourne ${ }^{2}$, F. Dominici ${ }^{1}$, L.A. Cooper ${ }^{1} ;{ }^{1}$ Johns Hopkins University, Baltimore, MD; ${ }^{2}$ RAND, Santa Monica, CA; ${ }^{3}$ University of Arkansas, Little Rock, AR (Tracking ID \#74770)

BACKGROUND: Patient involvement in decision-making (IDM) is an important component of patient centeredness, a key dimension of quality of care. Little empirical evidence exists to support that IDM plays a role in health outcomes, however. Our goal in this study was to determine the relationship between patients' ratings of their level of IDM and their symptoms of depression over 18 months of follow-up.

METHODS: Data are from 4 randomized clinical trials of quality improvement strategies for major depression in primary care. Patients were recruited from primary care offices across the US and were eligible if they were $\geq 18$ years old; had 2 or more weeks of depressed mood or persistent depression in the past year;and at least 1 week of depression in the past 30 days, as assessed by the Composite International Diagnostic Interview. Patients' scores on a modified version of the Center for Epidemiologic Studies (CESD) depression scale were assessed at 0, 6, 12,18 and 24 months. CESD scores could range from $0-100$, with $>20$ considered consisten with depression. Patients answered, "Rate your involvement in decisions about your care", using a 5 -point scale $(1=$ Poor, $5=$ Excellent $)$, with their usual provider at 6 months. To determine the relationship between ratings of IDM and CESD scores at $6,12,18$, and 24 months, we used a linear regression model with random intercept and generalized estimating equation (GEE) that controlled for sociodemographic data, baseline depression, intervention status, study site, antidepressant use, and the physical component scale score from the SF-12. RESULTS: 1661 patients were eligible for this analysis. Of them, $71 \%$ were women; the mean (SD) age was 44 (15) years; $57 \%$ of the sample was non-Hispanic white, $30 \%$ were Hispanic; and 9\% African-American. Patients' IDM ratings ranged 1-5, with mean 3.32 and SD 1.2. In bivariate regression, a 1-point increase in the patients' ratings of IDM corresponded to a drop in CESD score of 3.8 points on average, $\mathrm{p}<0.001$. In multivariable regression, a 1-point increase in IDM corresponded to a drop in CESD scores of 2.0 points on average over 18 months of follow-up, $\mathrm{p}<0.001$. This was the most substantial predictor of CESD score in our model. CONCLUSION: Patients' ratings of their involvement in decision making is substantially and significantly associated with improvements in their depression scores at up to 18 months from the time at which the IDM ratings were obtained. Interventions to increase patient participation in decision-making may be a promising strategy to improve outcomes for primary care patients with depression.

SURGICAL DECISIONS FOR EARLY STAGE NON-SMALL CELL LUNG CANCER: A PROSPECTIVE PILOT STUDY. S. Cykert ${ }^{1}$, T.S. Carey ${ }^{2}$; ${ }^{1}$ University of North Carolina at Chapel Hill, the Sheps Center for Health Services Research, and the Greensboro AHEC / Moses Cone Hospital, Greensboro, NC; ${ }^{2}$ University of North Carolina at Chapel Hill and Sheps Center for Health Services Research, Chapel Hill, NC (Tracking ID \#75123)

BACKGROUND: Several reports derived from administrative data have shown that 25 to $35 \%$ of patients diagnosed with early stage non-small cell lung cancer do not undergo potentially curative lung resection surgery and that African-Americans proceed to surgery less often than whites. The objective of this pilot study was to examine the process resulting in decisions for and against surgery and to demonstrate the ability to prospectively recruit patients during this stressful period.

METHODS: We identified patients with newly diagnosed non-small cell lung cancer in 3 ways: (1) by reviewing logs of a university thoracic oncology clinic, (2) by physician referral from community practices, and (3) by reviewing chest CT scans of patients who gave signed permission at any of 3 community hospitals. Patients were enrolled if they were above $18 \mathrm{yrs}$ of age, not yet scheduled for surgery, and thought to have clinical stage I or II non-small cell cancer. After we obtained informed consent, a survey was verbally administered to participating patients. The survey included questions regarding demographic data, health status, physicianpatient communication, trust, spirituality, and perceived prognosis. Also, we performed a limited chart review to ascertain co-morbid conditions and pulmonary function test results. RESULTS: Of the 29 patients enrolled in this pilot project, 22 were white and 6 AfricanAmerican. Sixteen patients were male, all were insured, and the mean age was $66.6 \mathrm{yrs}$. Nineteen of twenty-nine patients underwent lung resection surgery (14 of 22 whites; 5 of 6 African-Americans). The average \%-predicted FEV-1 in the surgery group was 69 compared to 81 in the no surgery group. The 2 patients with FEV-1 below $50 \%$ of predicted did not undergo surgery. In bivariate analysis, we found that the only statistically significant associations with the "no surgery" decision were dyspnea with minimal exertion $(\mathrm{p}=.005)$ and a less than $90 \%$ certainty in the belief of the lung cancer diagnosis $(\mathrm{p}=.006)$. Diabetics as well as patients who believed that prayer could cure cancer demonstrated a nearly significant trend to decide against surgery.

CONCLUSION: This prospective pilot study demonstrated that patients with early stage non-small cell lung cancer can be reasonably recruited. Early data revealed that disbelief of diagnosis is a major obstacle to surgery that could be amenable to intervention.

DISSEMINATION OF INFORMATION TECHNOLOGY IN HOSPITALS TO IMPROVE QUALITY OF CARE. B.N. Doebbeling ${ }^{1}$, T.E. Vaughn ${ }^{2}, \mathrm{~K} \mathrm{McCoy}^{3}$, B.J. BootsMiller ${ }^{3}$, S. Flach ${ }^{4}$, M.M. Ward ${ }^{2}$, C.J. Franciscus ${ }^{4}$, V. Forman ${ }^{4}$, P. Peloso ${ }^{4},{ }^{1}$ University of lowa Carver College of Medicine and the lowa City Veterans Affairs Medical Center, lowa City, IA ${ }^{2}$ University of lowa College of Public Health, lowa City, IA; ${ }^{3}$ lowa City Veterans Affairs Medical Center, lowa City, IA; ${ }^{4}$ University of lowa Carver College of Medicine, lowa City, IA (Tracking ID \#76675)

BACKGROUND: The Institute of Medicine (IOM) Crossing the Quality Chasm report recommended greater use of information technology (IT) to support improved quality of care. We seek to identify organizational factors that influence greater use of IT to improve patient care.

METHODS: We surveyed 4227 (58\% response rate) VA clinicians (physicians, nurse practitioners, PAs and registered nurses) from a stratified random national sample. Clinicians assessed their hospital's provision of IT support on Likert scales (not at all $=1$ to very great $=5$ ) across six dimensions: 1) access to literature/evidence, 2) decision support, 3) clinical data, 4) reduction of medical errors, 5) communication between providers, and 6) communication with patients. Factor analysis demonstrated a single factor (alpha $=0.76$ ). The dependent measure, IT clinical support, summed these items. Predictor variables in three domains, organizational context, change management and quality improvement culture, came from two other surveys: 
2000 AHA Survey and our 2001 quality manager survey of all VA hospitals (90\% responding). Generalized estimating equations regression estimated the importance of predictors in each domains for IT clinical support.

RESULTS: After combining data sets responses from 1752 providers at 122 hospitals were included in the analyses. Provider groups assessed IT clinical support slightly differently, although the effect size was small (0.06). Scores for the IT dimensions ranged from high for 1) computerized patient clinical data (mean 4.3); and 2) electronic communication between providers (mean 4.1); to moderate for 3 ) automation of decisions to reduce errors (mean $=3.8$ ) 4) access to literature/evidence based medicine while delivering care (mean 3.4); and 5) decision support systems (mean 3.3); to low for 6) electronic communication with patients (mean 2.5). IT clinical support was positively associated with urban location $(\mathrm{p}<0.05)$, and cooperative culture $(\mathrm{p}<0.01)$. Teaching status, personnel and network influence approached significance. Staffing ratios, hospital type (general medical-surgical vs. psychiatric or "other"), and change management measures were unrelated to IT clinical support.

CONCLUSION: Although clinicians report that the VA has widely implemented an electronic medical record and email communication among providers, other uses of IT to support improved quality of care vary. Clinicians rated urban hospitals and those with more cooperative cultures as providing high levels of IT clinical support. Further study is needed to determine which IT system dimensions contribute most to improved quality and how to effectively implement them.

BARRIERS TO USE OF CLINICAL PRACTICE GUIDELINES: PRE-IMPLEMENTATION DATA FROM THE ATHENA PROJECT. M.A. Fischer ${ }^{1}$, M.A. Steinman ${ }^{2}$, H.B. Bosworth ${ }^{3}$, E.Z. Oddone ${ }^{3}$, M. Shlipak ${ }^{2}$, M.K. Goldstein ${ }^{4} ;{ }^{1}$ University of Massachusetts, Worcester, MA; ${ }^{2}$ SF VAMC and UCSF, San Francisco, CA; ${ }^{3}$ Durham VAMC and Duke University, Durham, NC; ${ }^{4}$ Palo Alto VAMC and Stanford University, Palo Alto, CA (Tracking ID \#75922)

BACKGROUND: To determine how self-reported barriers to clinical practice guidelines (CPG) are associated cross-sectionally with clinician adherence to hypertension guidelines. METHODS: We surveyed 223 primary care clinicians at 3 VA medical centers regarding attitudes toward CPGs and perceived barriers to use of CPGs in 7 previously described domains (awareness, familiarity, agreement, self-efficacy, outcome expectancy, inertia, external). Using multivariate techniques that adjusted for clinician demographic and practice characteristics, we evaluated the association between self-reported barriers to CPG use and objective measures of guideline adherence obtained from VA clinical databases: (a) $>65 \%$ of patients using guideline-adherent anti-hypertensive agents, and; (b) $>55 \%$ of patients achieving adequate blood pressure control. Dichotomization was based on VA performance standards, adjusted to achieve comparably sized groups.

RESULTS: Of 137 respondents to the survey, clinical data on the clinician's panel of patients were available for $86(63 \%)$. Clinicians had favorable attitudes towards CPGs:only $1 \%$ reported negative attitudes toward JNC VI, whereas $70 \%$ claimed to seek guidelines for help with patient care. Prevalence of perceived barriers to use of CPGs varied greatly: $9 \%$ were unfamiliar with CPG-recommended treatments, $15 \%$ reported not having time to use CPGs, $27 \%$ did not believe that CPGs fit their patients, 36\% reported lack of awareness of existing CPGs, and $50 \%$ noted that CPGs are hard to find. Overall, $66 \%$ of patients were treated with guideline-concordant anti-hypertensives, whereas only $43 \%$ of patients met blood pressure targets. Clinician characteristics, practice characteristics, and attitudes toward CPGs were not associated with clinicians' use of guideline-recommended medications. In contrast, clinicians who were more aware of CPGs were substantially more likely to meet the target for patient BP control (OR 5.72, 95\% CI 1.08-30.27).

CONCLUSION: Clinicians with greater self-reported awareness of CPGs were more likely to meet BP control targets. Providing timely, educational CPG-based interventions at the pointof-care may capitalize on favorable attitudes toward CPGs while overcoming the barrier of awareness and improving BP control.

HOW CAN WE IMPROVE COMPLIANCE WITH A CONGESTIVE HEART FAILURE GUIDELINE? S. Flach ${ }^{1}$, K. Welke ${ }^{1}$, K. McCoy ${ }^{2}$, B.J. BootsMiller ${ }^{2}$, T.E. Vaughn ${ }^{3}$, M.M. Ward ${ }^{3}$, J. Perlin ${ }^{4}$, B.N. Doebbeling;: ${ }^{1}$ University of lowa Carver College of Medicine, lowa City, IA; ${ }^{2}$ Iowa City Veterans Affairs Medical Center, lowa City, IA; ${ }^{3}$ University of lowa College of Public Health, lowa City, IA; ${ }^{4}$ Department of Veterans Affairs Office of Quality and Performance, Washington, DC; ${ }^{5}$ University of lowa Carver College of Medicine and the lowa City Veterans Affairs Medical Center, lowa City, IA (Tracking ID \#76706)

BACKGROUND: Compliance with clinical practice guidelines (CPGs) is often sub-optimal We seek to describe compliance with a recently implemented congestive heart failure (CHF) guideline and determine the relationship between organizational practices and beliefs concerning CPGs and adherence to the guideline.

METHODS: We used a 2001 survey of quality managers, individuals who supervise CPG implementation, at all 143 Veterans Affairs (VA) hospitals to obtain information about practices their hospital undertook to implement CPGs and perceived attitudes about CPGs. We used the 2001 External Peer Review Program (EPRP) of chart audits of CHF patients to obtain our dependent variable, a hospital-level benchmarking score reflecting CHF CPG adherence. The benchmarking score used a previously validated and published method to describe overall hospital performance based on the chart audit results concerning seven key CHF CPG recommendations (eg, ACE inhibitor use, LV assessment). The score was obtained by summing the number of times the hospital's performance exceeded the 50th (one point) and 75 th (two points) percentile benchmark compared with other hospitals for each of the seven guideline components. We used multivariate linear regression to assess the relationship between the explanatory variables and the hospital-level CHF benchmarking score.

RESULTS: Results from 126 (88\%) VA hospitals indicated variable adherence to the guideline, with $14 \%$ of patients receiving adequate dietary and medication instruction, and $91 \%$ receiving measurement of $L V$ function. Variables associated $(\mathrm{P}<0.05)$ with improved facility level adherence included: teaching hospital status, better patient population health status, improved teamwork when implementing CPGs, and the establishment of checkpoints and deadlines. A passive implementation method, use of instructional audio/video tapes, and collecting other clinical outcome data, were associated with diminished adherence.

CONCLUSION: Compliance with a CHF guideline varies. Our study identifies specific areas, particularly the level of teamwork and establishment of checkpoints and deadlines when implementing CPGs, which provide opportunities to improve adherence to a CHF guideline. Efforts to improve $\mathrm{CHF}$ guideline adherence should concentrate on those implementation and monitoring approaches shown to be most effective.

ADVERSE DRUG EVENTS FOLLOWING HOSPITAL DISCHARGE. A.J. Forster ${ }^{1}$, D.W. Bates ${ }^{2}$, T. Gandhi ${ }^{2}$, H. Murff ${ }^{2}$, J. Peterson ${ }^{2}$; ${ }^{1}$ Ottawa Health Research Institute, Ottawa, Ontario; 'Brigham and Women's Hospital, Boston, MA (Tracking ID \#75754)

BACKGROUND: We recently reported that $19 \%$ of medical patients are affected by adverse events shortly following discharge. We also determined that adverse drug events (ADEs) were the most common class of adverse event (Annals of Internal Medicine, 02/04/03). We present the results of a secondary analysis of this data to evaluate post-discharge ADEs in more detail. Specifically, we sought to describe the incidence of ADEs, preventable ADEs and ameliorable ADEs occurring after discharge and their associated risk factors.

METHODS: Consecutive patients discharged from the general medical service of a large urban academic hospital were eligible. We reviewed the inpatient record and performed a telephone interview approximately 24 days after discharge. The purpose of the interview was to identify new or worsening symptoms, the patient's health system use, and their recollection of processes of care. We combined the interview and hospital record information into a case summary, which two internists reviewed independently. Using implicit criteria, they rated the case summary to determine $\mathrm{ADE}$ occurrence, as well as to rate preventability, ameliorability and severity.

RESULTS: We studied 400 patients within 5 weeks of discharge. Of these, 45 patients developed an ADE following discharge (11\% [95\% CI, $8 \%$ to $14 \%]$ ). $27 \%$ of ADEs were preventable and $33 \%$ were ameliorable. Injuries were significant in 32 patients, serious in six, and life-threatening in seven. Patients receiving more prescriptions were more likely to have an $\mathrm{ADE}$. The risk of $\mathrm{ADE}$ in the group of patients receiving the highest quartile of prescriptions was significantly higher than the group receiving the lowest ( $\mathrm{OR}=2.8$ [95\% CI, 1.2 to 6.5$]$ ). The risk of ADE per prescription was high for corticosteroids ( $7 \%$ [95\% CI, $2 \%$ to $12 \%]$ ), anticoagulants ( $7 \%$ [ $95 \% \mathrm{CI}, 0 \%$ to $14 \%]$ ), antibiotics (5\% [95\% CI, 3\% to $8 \%]$ ), analgesics (3\% [ $95 \% \mathrm{CI}, 0 \%$ to $6 \%]$ ), and cardiovascular medications ( $1 \%$ [ $95 \% \mathrm{CI}, 0 \%$ to $3 \%]$ ). Patients were less likely to experience an ADE if they recalled having had the side effects of prescribed medications explained ( $\mathrm{OR}=0.4$ [95\% CI, 0.2 to 0.8]). Patient age, sex, marital status, Charlson index and insurance type were not associated with $\mathrm{ADE}$ occurrence.

CONCLUSION: Following discharge, ADEs are common and many of them are preventable or ameliorable. Interventions to improve medication safety could target patients receiving specific drug classes or multiple medications. Medication side effects should be discussed and reinforced with patients.

AUTOMATED WARNINGS TO REDUCE INPATIENT ADMINISTRATION OF METFORMIN IN PATIENTS WITH RENAL INSUFFICIENCY. W.L. Galanter ${ }^{1}$, A. Polikaitis ${ }^{2} ;{ }^{1}$ University of Illinois at Chicago, Chicago, IL; ${ }^{2}$ Cerner Corporation, Kansas City, MO (Tracking ID \#74069)

BACKGROUND: Many adverse drug events are associated with the inappropriate use of renally cleared medications in patients with renal dysfunction. Automated clinical decision support for the safe use of renally excreted medications has potential to reduce such medication errors and ultimately adverse drug events. We report on a trial of an automated decision support intervention designed to alert prescribers during computerized physician order entry (CPOE) and thereby reduce the inpatient use of medications contraindicated due to renal dysfunction. METHODS: An automated decision support alert intervention was designed utilizing a commercially available clinical decision support engine and electronic medical record (Discern Expert and Millennium, Cerner Corporation). The warning of contraindication was based on an estimate of the creatinine clearance calculated from patient-specific data available in the electronic medical record (age, gender, serum creatinine, and height, if present). The drug metformin was used as a model as it was the most frequently prescribed of the potentially contraindicated drugs. The administration of metformin to inpatients with creatinine clearances less than $60 \mathrm{ml} / \mathrm{min}$ was analyzed for a 6-month study period after alert implementation as well as for a 4-month historical cohort immediately prior to alert implementation. The outcome measure was the likelihood of a patient receiving at least one dose of the medication after order initiation within CPOE.

RESULTS: The number of metformin orders initiated within CPOE for patients with an estimate creatinine clearance less than $60 \mathrm{ml} / \mathrm{min}$ in the control and study groups were 62 and 144 . The average creatinine clearances in the control and study groups were $47.2 \pm 10.2 \mathrm{ml} / \mathrm{min}$ and $45.3 \pm 11.7 \mathrm{ml} / \mathrm{min}(\mathrm{P}=0.27$ for difference). The likelihood of receiving a dose of metformin with an estimated creatinine clearance less than $60 \mathrm{ml} / \mathrm{min}$ in the control group was $92 \%$. In the study group, the likelihood was reduced to $53 \%(\mathrm{P}<0.0001)$ as many clinicians followed the alert recommendation and discontinued the metformin order.

CONCLUSION: Automated decision support alerts displayed to clinicians during CPOE were effective in reducing the inpatient use of metformin in patients with renal dysfunction. The efficacy of this alert intervention was limited by clinician non-compliance to the alert recommendations. Further analysis of the reasons for clinician non-compliance is necessary to improve the alert efficacy.

ANALYSIS OF COMPLIANCE WITH AUTOMATED DECISION SUPPORT ALERTS FOR CONTRAINDICATED MEDICATIONS USING COMPUTERIZED PHYSICIAN ORDER ENTRY (CPOE) W.L. Galanter ${ }^{1}$, R. Mrtek ${ }^{1}$, A. Polikaitis ${ }^{2} ;{ }^{1}$ University of Illinois at Chicago, Chicago, IL; ${ }^{2}$ Cerner Corporation, Kansas City, MO (Tracking ID \#74790)

BACKGROUND: Automated clinical decision support has shown promise in reducing medication errors, however clinicians often do not comply with the recommendations. An 
understanding of the factors which impact compliance in the CPOE environment is important as many healthcare systems are planning implementation of $\mathrm{CPOE}$. The benefit of providing alerts in real-time, via on-screen displays during CPOE may be mitigated by non-compliance. We report on factors related to compliance with alert recommendations designed to reduce inpatient use of medications contraindicated due to renal dysfunction in a CPOE environment. METHODS: Alerts were designed using a commercially available clinical decision support engine. The warning of contraindication was based on an estimate of the creatinine clearance calculated from available patient data. A minimum safe creatinine clearance was established for each medication by the pharmacy. Real-time alerts appeared when a contraindicated order was initiated, recommending cancellation and providing the patient's creatinine clearance as well as the minimum safe creatinine clearance. Inpatient ordering of medications in patients with creatinine clearances less than their minimum safe level was studied for 6 months after implementation. The outcome was compliance with alert recommendation. The impact of several factors on compliance was examined using Spearman correlation coefficients with $\mathrm{P}$ set at 0.05 .

RESULTS: Clinicians complied with $40 \%$ of the alerts. Compliance with alert recommendations was found to be associated with the degree of renal dysfunction $(\mathrm{P}=0.002)$. Days were split into 2 time periods, 7AM to 7PM $(\mathrm{n}=117)$ and $7 \mathrm{PM}$ to $7 \mathrm{AM}(\mathrm{n}=52)$. Alert compliance during the overnight period was greater than during the day, $52 \%$ vs. $34 \%, P=0.03$. Housestaff with more than 1 year of training $(\mathrm{n}=61)$ were found to be more compliant with the alert recommendations than those with less than 1 year of training $(n=66), 51 \%$ vs. $32 \%, P=0.03$. CONCLUSION: Compliance with the alerts was found to be a function of the training of the ordering house officers as well as the time of day. Compliance was also found to vary with the degree of renal dysfunction of the patient. The patient's estimated creatinine clearance was provided in the alert message, suggesting that clinicians do actually consider the alert recommendation before making a clinical decision. Additional studies are necessary to fully understand how clinical decision support systems can optimally interact with clinicians.

PATIENTS' AND PHYSICIANS' ATTITUDES REGARDING THE DISCLOSURE OF MEDICAL ERRORS. T.H. Gallagher ${ }^{1}$, A.D. Waterman ${ }^{2}$, A.G. Ebers ${ }^{2}$, V.J. Fraser ${ }^{2}$, W. Levinson ${ }^{3}$; ${ }^{1}$ University of Washington, Seattle, WA; ${ }^{2}$ Washington University, St. Louis, $\mathrm{MO} ;{ }^{3}$ University of Toronto, Toronto, Ontario (Tracking ID \#75290)

BACKGROUND: Despite our best efforts, medical errors are inevitable. While disclosing errors to patients is recommended, little is known about how patients and doctors think medical errors should be discussed.

METHODS: We conducted 13 focus groups to determine patients' and physicians' attitudes about medical error disclosure. Six groups contained patients, four groups contained physicians, and three groups included both doctors and patients. 52 patients and 46 physicians participated in the groups. Qualitative analysis was conducted on the focus group transcripts. RESULTS: Both patients and physicians had unmet needs following errors. Patients wanted disclosure of all harmful errors, and sought information about what happened, why the error happened, how the error's consequences will be mitigated, and how recurrences will be prevented. Physicians agreed that harmful errors should be disclosed, but "choose their words carefully" when telling patients about errors. While physicians disclosed the adverse event, they often avoided stating that an error occurred, why the error happened, or how recurrences would be prevented. Patients also desired emotional support from physicians following errors, including an apology. However, physicians worried that an apology might create legal liability. Physicians were also upset when errors happen, but were unsure where to seek emotional support.

CONCLUSION: Physicians may not be providing the information or emotional support that patients seek following harmful medical errors. Physicians should strive to meet patients' desire for an apology and for information on the nature, cause, and prevention of errors. Institutions should also address the emotional needs of providers who are involved in medical errors.

IDENTIFICATION OF OUTPATIENT ADVERSE DRUG EVENTS USING A COMPUTER MONITOR. T. Gandhi ${ }^{1}$, J.M. Overhage ${ }^{2}$, A.C. Seger ${ }^{1}$, C. Hope $^{2}$, M.D. Murry ${ }^{2}$, D. Weber ${ }^{1}$, J. Fiskio ${ }^{1}$, E. Teal ${ }^{2}$, V. Mills ${ }^{2}$, D.W. Bates ${ }^{1}$; ${ }^{1}$ Division of General Medicine, Brigham and Women's Hospital, Boston, MA; ${ }^{2}$ Indiana University/Regenstrief Institute, Indianapolis, IN (Tracking ID \#75771)

BACKGROUND: Identifying adverse drug events (ADEs) in the ambulatory setting is difficult. Review of paper charts is costly and is not routinely done. We sought to develop a computerized ADE monitor that could identify ADEs in a systematic way at clinics in 2 large academic health centers.

METHODS: We developed a computerized ADE monitor, a program consisting of 129 rules (such as drug-lab and drug-drug combination rules), that can be applied to outpatient electronic medication and lab data to identify possible ADEs. The possible ADEs identified are reviewed using medical records to determine true positives (true ADEs). For example, a rule would look for patients with increased serum creatinine who are taking metformin. Patients identified by the rule would have their chart reviewed to look for an episode of lactic acidosis (an ADE). Outpatient rules were developed from literature review and expert opinion. We applied the ADE monitor to 4 months of outpatient data (1/01-5/01) from 11 Boston-area sites and 22 Indianapolis-area sites. Per rule, a maximum of 50 possible ADEs were randomly selected for review. Positive predictive values (PPV) were then calculated based on projections to the total possible ADEs identified.

RESULTS: The monitor's PPV for ADEs was . 10 in Boston and .11 in Indianapolis. Projected $\mathrm{ADE}$ rates and preventable ADE rates for our sample are shown in Table 1. PPVs were highest for lab-related rules (.15 and .22 respectively).

In Boston, $5 \%$ of true ADEs were life threatening and $24 \%$ were serious. An example of a life threatening ADE was a high potassium due to an ACE inhibitor. An example of a serious ADE was bradycardia due to a beta blocker.

CONCLUSION: The PPV of the ADE monitor was consistent between the two organizations. Interestingly, ADE rates were different which could reflect differences in clinical practice, documentation, and/or patients. This study demonstrates that an $\mathrm{ADE}$ monitor can be implemented in different organizations and can be an efficient way to utilize electronic medical data to identify ADEs, particularly those related to lab test issues.

THE MANAGEMENT OF BREAST CANCER IN HAWAII: A SURVEY OF HEALTH CARE PRACTICES. R.P. Gelber ${ }^{1}$, T.B. Seto ${ }^{2}$; ${ }^{1}$ University of Hawaii John A. Burns School of Medicine, Honolulu, HI; ${ }^{2}$ The Queen's Medical Center, Honolulu, HI (Tracking ID \#75445)

BACKGROUND: Despite recommendations such as those from the NCI/NIH, there is evidence that physicians vary in their treatment (tx) recommendations for women with early stage breast cancer $(\mathrm{Ca})$. This discrepancy appears to be associated with race and socioeconomic status, although the reasons for this variation are unclear. We developed a survey to assess how physicians treat pts with early stage breast $\mathrm{Ca}$ and compared their responses to national recommendations.

METHODS: We mailed surveys to all 81 physicians in $\mathrm{HI}$ who treat pts with breast Ca. Surveys included 6 questions on tx of stages I/II, with answers based on clear recommendations from the NCI/NIH. We asked 6 questions on management issues addressed in NCI/NIH publications but not strongly endorsed for lack of consensus, including axillary node staging/ sentinel node biopsy, timing of mammography and referral to a plastic surgeon following Ca resection. Physicians were asked if they believe pt race affects tolerance of therapy and tx recommendations. Surveys were mailed in 11/02 with a follow-up mailing after $1 \mathrm{mo}$.

RESULTS: The 81 physicians who were mailed the survey included 28 medical oncologists, 9 radiation oncologists, 3 surgical oncologists, 41 general surgeons. $72 \%$ (58) of physicians returned the survey. $91 \%$ of the questions on tx recommendations were answered correctly, in accordance with NCI/NIH publications. Of these, $94 \%$ of questions pertaining to premenopausal women were answered correctly, compared to $87 \%$ of those regarding postmenopausal women $<70 \mathrm{yrs}(\mathrm{p}=0.03)$. Slightly more responses pertaining to Stage I were correct compared to Stage II (93\% vs. $89 \%$ ). All responders recommended mammography of the contralateral breast within 18 mo of tumor resection. In contrast, $69 \%$ reported that they routinely recommend axillary lymph node dissection for staging, and $48 \%$ recommend sentinel lymph node biopsy. Only $26 \%$ routinely refer their pts to a plastic surgeon following mastectomy. $14 \%$ believed pts' race affects their tolerance of adjuvant chemotherapy, whereas $3 \%$ believed race affects tolerance of radiation therapy. None reported that race affects tx recommendations.

CONCLUSION: The majority of physicians in Hawaii who treat patients with breast Ca reported practice patterns in accordance with national guidelines. Consistent with the lack of consensus in NIH/NCI publications, physicians responded with substantially more variation to questions concerning issues that are less clearly defined. None of the physicians reported that patient race affects treatment recommendations.

USE OF VENOUS THROMBOEMBOLISM PROPHYLAXIS IN HIGH-RISK PATIENTS. R.P. Gelber ${ }^{1}$, Y. Chen ${ }^{2}$, T.B. Seto ${ }^{2}$; ${ }^{1}$ University of Hawaii John A. Burns School of Medicine, Honolulu, HI; ${ }^{2}$ The Queen's Medical Center, Honolulu, HI (Tracking ID \#75457)

BACKGROUND: Patients who undergo hip fracture surgery (HFS) and total hip (THR) or knee (TKR) replacement are at high risk for venous thromboembolism (VTE). The 6th ACCP Antithrombotic Therapy guidelines recommend LMWH or warfarin as VTE prophylaxis for these patients. Despite the publication of these guidelines and established efficacy of their recommended therapies, the use of VTE prophylaxis in these high-risk patients is unknown. METHODS: We identified pts who underwent HFS, THR, or TKR at our institution from 9/ 98 to 6/02. Data obtained included type/frequency of VTE prophylaxis (LMWH, heparin, aspirin, warfarin, intermittent pneumatic compression [IPC]), lab data, wt, demographics, type of anesthesia. VTE risk factors (eg, cancer, prior VTE) and potential contraindications to prophylaxis (eg, liver disease, hemorrhage) were identified through discharge diagnoses. Based on ACCP guidelines and our hospital formulary, we identified 'Recommended' VTE prophylaxis as 1) enoxaparin $30 \mathrm{mg}$ SQ bid started $12-24$ hrs post-op or $40 \mathrm{mg}$ SQ qd started 10-12 hrs pre-op; 2) warfarin (INR 2-3) started pre-op or immediately post-op; 3) IPC started pre-op.

RESULTS: Of 2047 pts, $56 \%$ were women, $44 \%$ white, $22 \%$ Japanese, $12 \%$ Pacific-Islanders. Mean age was $68 \pm 18$, with $51 \%>70$ yrs old. $26 \%$ (531) underwent HFS, $40 \%$ (817) THR, $34 \%$ (699) TKR. 38\% received spinal or epidural anesthesia. $22 \%$ (456) had $\geq 1$ additional VTE risk factor, including $17 \%$ (338) with obesity. $9 \%$ (187) had potential contraindications to VTE prophylaxis, including preexisting bleeding disorder, plt $<100,000, \mathrm{Cr}>2.0$. Overall, $35 \%$ (717) received recommended VTE prophylaxis, including $15 \%$ (79) of HFS, 39\% (320) of THR, and $45 \%$ (318) of TKR pts. $22 \%$ (447) received non-recommended prophylaxis, including $18 \%$ who received aspirin alone. $43 \%$ did not have any prophylaxis. Japanese pts were significantly less likely to receive VTE prophylaxis than other ethnicities (31\% vs. $36 \%$, $\mathrm{p}=0.04)$. Pts with spinal/epidural anesthesia were significantly less likely than general anesthesia pts to receive recommended therapy ( $28 \%$ vs. $37 \%$ ). Excluding pts with potential contraindications to prophylaxis $(9 \%)$ or regional anesthesia, only $37 \%$ received appropriate VTE prophylaxis.

CONCLUSION: Despite the development of national guidelines, such as those from the 6th ACCP, the use of VTE prophylaxis remains low, with only $35 \%$ of high-risk patients receiving recommended therapy. Further research is needed to clarify the possible reasons for our findings, including pt preferences, contraindications to therapy, and physician awareness of optimal VTE prophylaxis.

HYPERTENSION TREATMENT PRACTICES OF CLINICIANS IN EAST AND CENTRAL HARLEM. C.E. Goldstein ${ }^{1}$, P. Hebert ${ }^{1}$, C.R. Horowitz ${ }^{1}$, M.A. McLaughlin ${ }^{1}$, T.G. McGinn ${ }^{1}$, J.E. Sisk ${ }^{1} ;{ }^{1}$ Mount Sinai School of Medicine, New York, NY (Tracking ID \#74614)

BACKGROUND: Despite benefits in the treatment of hypertension and well established treatment guidelines, hypertension control rates have not improved in recent years. One possible explanation is that clinicians lack awareness of recommended blood pressure (BP) 
levels for patients with common comorbidities. We surveyed clinicians at all four hospitals in East and Central Harlem in New York City to assess their therapeutic goals regarding the treatment of patients with uncontrolled chronic hypertension, and to ascertain whether patient sociodemographic characteristics influenced the physcian's choice of target blood pressure. METHODS: We developed four clinical vignettes of hypertensive patients. The BP values and comorbidities of each scenario were constant, but patient sociodemographic characteristics such as gender, age, race/ethnicity, and occupation were assigned randomly, such that each survey contained a distinct mix of patient demographics. We surveyed attending physicians, fellows, house officers and nurse practitioners in internal medicine, geriatrics and cardiology clinics associated with each hospital. On the basis of the guidelines from The Sixth Report of the Joint National Committee on Prevention, Detection, Evaluation, and Treatment of High Blood Pressure (JNC-6), we defined adequate BP control as BP $<140 / 90$ for isolated systolic hypertension (ISH) or coronary artery disease (CAD); $<130 / 85$ for diabetes, and $<125 / 75$ for renal disease (creatinine $\geq 2.0$ and $>1$ gram proteinuria).

RESULTS: To date, 180 of an anticipated 300 clinicians have completed surveys; $53 \%$ were women and $58 \%$ were house officers. For patients with renal disease only $2 \%$ of clinicians chose a target BP that was strictly compliant with guidelines, and $5 \%$ were within $2 \mathrm{mmHg}$ of the guideline. Compliance was better for the vignettes with diabetes, (37\% strictly compliant, $86 \%$ within $2 \mathrm{mmHg}$ ), ISH (70\% strictly compliant, $98 \%$ within $2 \mathrm{mmHg}$ ), and CAD (84\% strictly compliant, $99 \%$ within $2 \mathrm{mmHg}$ ). BP targets did not vary by patient sociodemographics, nor by clinician gender or experience.

CONCLUSION: Preliminary results suggest that the majority of clinicians surveyed chose BP targets that approximate the JNC-6 guidelines for ISH, CAD and diabetes. Most did not treat hypertensive patients with renal disease according to the guidelines. Clinician education on target BP levels in patients with renal disease is warranted.

WHEN DO PATIENTS AND THEIR PHYSICIANS AGREE ON DIABETES TREATMENT GOALS AND STRATEGIES, AND WHAT DIFFERENCE DOES IT MAKE? M. Heisler ${ }^{1}$, S. Vijan ${ }^{1}$, R.M. Anderson ${ }^{1}$, P.A. Ubel ${ }^{1}$, S.J. Bernstein ${ }^{1}$, T.P. Hofer ${ }^{1}$; ${ }^{1}$ University of Michigan, Ann Arbor, Ml (Tracking ID \#74170)

BACKGROUND: For patients with chronic illnesses, it is hypothesized that effective patientprovider collaboration contributes to improved patient self-care by promoting greater agreement on patient-specific treatment goals and strategies. However, this hypothesis has not been tested in actual encounters of patients with their own physicians.

METHODS: We assessed the extent to which patients with type 2 diabetes agree with their primary care providers (PCPs) on diabetes treatment goals and strategies, the factors that predict agreement, and whether greater agreement is associated with better patient selfmanagement of diabetes. 127 pairs of patients and their PCPs in two health systems were surveyed about their top three diabetes treatment goals (desired outcomes from diabetes treatment) and strategies to meet those goals. Using several measures to evaluate agreement, we explored whether patient characteristics, such as education and attitudes toward treatment, and patient-provider interaction styles, such as shared decision-making, were associated with greater agreement on treatment goals and strategies. We then examined whether that agreement was associated with higher assessments by the patients of their diabetes care selfefficacy and self-management.

RESULTS: Overall, agreement on top treatment goals and strategies was low (all kappas were less than 0.40). In multivariable analyses, however, patients with more education, greater belief in the efficacy of their diabetes treatment, and who shared in treatment decision-making with their providers were more likely to agree with their providers on treatment goals or strategies. Similarly, physician reports of having discussed more content areas of diabetes self-care were associated with greater agreement on treatment strategies. In turn, greater agreement on treatment goals and strategies was associated both with higher patient diabetes care selfefficacy and assessments of their diabetes self-management.

CONCLUSION: Although patients and their PCPs in general had poor agreement on goals and strategies for managing diabetes, better agreement was associated with improved patient self-efficacy and diabetes management. This supports the hypothesis that enhancing patientprovider agreement on both overall treatment goals and specific strategies to meet these goals may lead to improved patient outcomes.

EFFECTIVENESS OF CARE MANAGEMENT FOR PRIMARY CARE PATIENTS WITH PANIC DISORDER. B. Herbeck Belnap ${ }^{1}$, W.P. Gardner ${ }^{1}$, C.F. Reynolds ${ }^{1}$, K. Shear ${ }^{1}$, B.L. Rollman ${ }^{1}$; ${ }^{1}$ University of Pittsburgh, Pittsburgh, PA (Tracking ID \#76854)

BACKGROUND: Panic disorder (PD) and depression are common among primary care patients and frequently co-morbid. Both disorders are typically under-recognized and inadequately treated. Telephone-based collaborative care for the treatment of depression in primary care has been proven effective. However, little is known about the effectiveness of this strategy in treating patients with PD.

METHODS: Primary care patients reporting to four different practices were screened for PD and depression with the PRIME-MD. Patients who met severity criteria (Panic Disorder Severity Scale (PDSS) >6) were randomized to either a 12-month telephone-based collaborative care management for treatment of $\mathrm{PD}$, or to a "usual care" control condition. Intervention patients could choose any combination of the following treatments for $\mathrm{PD}$ : pharmacotherapy, use of a self-management workbook under the direction of the care manager, or specialty referral. Blinded telephone assessments of anxiety (PDSS) and depression (Hamilton Rating Scale for Depression (HRS-D)) were conducted at baseline and at 2, 4, 8, and 12 months following recruitment.

RESULTS: 102 patients met severity criteria for PD. Their mean age was 42 (range 19-63), $80 \%$ were female, $97 \%$ Caucasian and $68 \%$ were identified with co-morbid depression. At baseline, patients with co-morbid depression scored higher than those without depression on the PDSS (11.6 vs. 9.6; $\mathrm{p}=0.002)$ and HRS-D (19.9 vs. 10.4; $\mathrm{p}<0.0001)$, but were similar in sociodemographic variables. Random-effects regression analyses demonstrated that PD patients with co-morbid depression randomized to our intervention improved significantly on the PDSS compared to usual care ( $\mathrm{p}=0.01$ ), but not on the HRS-D after adjustment for baseline sociodemographic characteristics. However, patients with PD alone had a similar improvement regardless of randomization status.

CONCLUSION: Telephone-based collaborative care management for primary care patients with $\mathrm{PD}$ is more effective for more severely impaired individuals with co-morbid depression than for those with PD alone.

WHERE ARE INAPPROPRIATE MEDICATIONS PRESCRIBED TO OLDER PATIENTS? T. Higashi ${ }^{1}$, N.S. Wenger ${ }^{1} ;{ }^{1}$ University of California, Los Angeles, Los Angeles, CA (Tracking ID \#74354)

BACKGROUND: Prior studies show that a large number of inappropriate medications are prescribed to older patients, putting them at unnecessary risk of adverse drug events. To focus interventions to minimize the use of inappropriate drugs, it is important to understand the situations in which these drugs are provided.

METHODS: Prescriptions in a nationally representative sample of emergency department (ED) visits from the National Hospital Ambulatory Medical Care Survey (NHAMCS) in 1998-2000 were evaluated using a previously developed list of medications that should generally be avoided in the care of the elderly. Analysis focused on the subset of medications defined by geriatric experts as "always to be avoided" as well as on the entire list (termed "generally inappropriate"). Comparison was made to hospital outpatient department (OPD) visits and office-based physician visits derived from the National Ambulatory Medical Care Survey (NAMCS) of the same period. We also examined other factors associated with "always avoid" inappropriate medication prescriptions. Conservative $99 \%$ confidence intervals were used as recommended by the National Center for Health Statistics.

RESULTS: Among 10640 ED visits by persons age 65 years or older, representing 47 million visits nationally, $4.8 \%$ of visits resulted in prescription of at least one "always avoid" inappropriate drug (generally inappropriate drugs prescribed at $15.2 \%$ of visits). This was significantly higher than $1 \%$ of visits with "always avoid" drugs $(6.6 \%$ "generally inappropriate") in hospital OPDs and $0.8 \%$ of visits with "always avoid" drugs $(6.5 \%$ "generally inappropriate") for office-based physician visits $(\mathrm{P}<0.0001)$. Multivariate analysis showed that "always avoid" inappropriate prescribing among elderly patients occurred more commonly in whites $(\mathrm{OR}=2.08$ vs black, $99 \%$ CI $1.03-4.17)$, younger elders (age 65-69 $\mathrm{OR}=1.92$ vs age $85+, 99 \% \mathrm{CI} 1.06-3.45)$, in those who received more medications $(\mathrm{OR}=1.39$ per drug prescribed, $99 \% \mathrm{CI} 1.29-1.48)$, and in visits midnight to $8 \mathrm{AM}(\mathrm{OR}=1.85$ vs $8 \mathrm{AM}$ to 4PM, 99\% CI 1.18-2.91). EDs in private hospitals more often than government hospitals provided "always avoid" drugs to older patients whether the ownership was for-profit $(\mathrm{OR}=1.96,99 \% \mathrm{CI} 0.91-4.20)$ or not-for-profit $(\mathrm{OR}=1.67,99 \% \mathrm{CI} 0.90-3.08)$. There was no geographic or yearly differences during 1998 to 2000 in prescribing "always avoid" drugs. CONCLUSION: Interventions are needed to improve ED medication management for older patients, particularly in the early morning and in private hospital EDs.

MEASURING QUALITY OF CARE: IS THERE A ROLE FOR PEER REVIEW? T.P. Hofer ${ }^{1}$ S.M. Asch ${ }^{2}$, M. Hogan ${ }^{3}$, J. Adams ${ }^{2}$, E.M. Kerr'; ; ${ }^{1}$ University of Michigan, Ann Arbor, Ml; ${ }^{2}$ RAND, Los Angeles, CA; ${ }^{3}$ VA HSR\&D, Ann Arbor, MI (Tracking ID \#76590)

BACKGROUND: We sought to develop a more reliable structured implicit chart review instrument for use in assessing the quality of care for chronic disease. We hypothesized that we would be more successful for clinical conditions that have a substantial evidence base for treatment in the clinical literature. We also illustrate a method of analysis that allows one to determine the measurement questions to which the instrument is generalizable.

METHODS: As part of a larger study comparing implicit and explicit measures of quality across 26 sites in the VA, we developed a structured implicit review instrument for four clinical conditions: COPD, diabetes (DM), hypertension (HTN) and acute conditions. Twelve reviewers carried out 180 reviews of 60 medical records for the care given in up to all four of the conditions over a 13 month period, including inpatient and outpatient care. We estimated the reviewer, record and noise variance for the calculation of reliabilities in a multilevel analysis. RESULTS: The reliability of a single review for detecting differences in quality of hypertension care and diabetes care across patients is .45 . This estimate, which is generalizable to the population of reviewers and patients, is substantially higher than most prior estimates of implicit review reliability. However, for COPD and acute care, two clinical areas where the body of evidence supporting specific interventions is substantially less, the reliability is poor (.26 and .11 respectively). The lower reliabilities are due to decreased signal rather than increased noise. With a careful reliability study the reviewer effects can be removed raising the reliability for the review of DM and $\mathrm{HTN}$ as high as .53 .

CONCLUSION: It is possible to obtain reliable ratings of chronic disease and that higher reliability is achieved for reviews in clinical areas that have a more developed evidence base. With a less developed evidence base the reviewers appear to have more difficulty in making systematic distinctions in the quality of care between patients. This analytic approach allows for generalizable conclusions about the use of these instruments and reasons for low or high reliability. Sampling relatively few records for structured implicit review may be a reliable alternative or complement to assessing quality of care by checklist approaches.

QUALITY OF LIFE CHANGES IN MEN WITH LOCALIZED PROSTATE CANCER VERSUS CONTROLS. R.M. Hoffman ${ }^{1}$, S.N. Stone ${ }^{2}$, W.C. Hunt ${ }^{2}$, F.D. Gilliland ${ }^{3} ;{ }^{1} \mathrm{New}$ Mexico VA Health Care System, Albuquerque, NM; ${ }^{2}$ New Mexico Tumor Registry, Albuquerque, NM; ${ }^{3}$ University of Southern California, Los Angeles, CA (Tracking ID \#75580)

BACKGROUND: Radical prostatectomy (RP) and radiation therapy (XRT) are prostate cancer treatments that can impair urinary, sexual, and bowel function. However, these functions can also decline with aging. We evaluated men with localized prostate cancer and a matched control group to compare the effects of cancer treatment versus aging on healthrelated qualify of life. 
METHODS: Cases (CaP) were New Mexican men, participating in the population-based Prostate Cancer Outcomes Study diagnosed with clinically-localized prostate cancer between 1994 and 1995. We randomly selected controls (C) from Motor Vehicle Department records and Health Care Finance Administration claims files, frequency matching them on five-year age groups and ethnicity. All subjects were surveyed by mail at baseline and after 5 years; data were collected on demographics, socioeconomic status, comorbidity, SF-36 domains (pain, physical, emotional, vitality, and mental health) and urinary, sexual, and bowel function. Mean composite scores for these functions ranged from 0 (poorest) to 100 (highest). We compared cases and controls using a generalized logit model.

RESULTS: We evaluated 344 cases ( 235 treated with RP, 61 with XRT) and 618 controls. At baseline, $\mathrm{CaP}$ had higher sexual function than $\mathrm{C}(66.7$ vs. $61.7, \mathrm{P}<0.03)$. However, $\mathrm{CaP}$ had lower mean scores on the SF-36 physical role $(55.1$ vs. $62.2, \mathrm{P}<0.05)$ and emotional role $(70.1$ vs. $77.9, \mathrm{P}<0.02)$ scales and higher mean scores on the pain scale $(76.0$ vs. $68.4, \mathrm{P}<0.001)$. There were no differences in urinary or bowel function, comorbidities, or overall health. After five years, sexual function declined significantly in both groups, but more in $\mathrm{CaP}(-31.6)$ than $\mathrm{C}(-5.6)$, between group $\mathrm{P}<0.0001$. Urinary function also significantly declined in $\mathrm{CaP}$ $(-14.7)$, but not $\mathrm{C}(-0.2)$, between group $\mathrm{P}<0.0001$. RP led to greater declines than XRT in urinary $(-16.1)$ and sexual $(-10.8)$ function. SF-36 general health domains did not significantly change for either group. The proportion of $\mathrm{CaP}$ reporting excellent health declined significantly $(20.1 \%$ to $16 \%, \mathrm{P}=<0.04)$, but was unchanged in $\mathrm{C}(16 \%$ to $17 \%)$.

CONCLUSION: Men with localized prostate cancer reported significant declines in urinary and sexual function by 5 years after diagnosis, especially following RP. The control group had a significant, but lesser decline in sexual function, and urinary function remained stable. Overall, general health-related quality of life was similar at follow-up for both groups.

TESTING THE POTENTIALLY PREVENTABLE COMPLICATIONS MODULE USING COMPUTERIZED HOSPITAL DISCHARGE DATA. J. Hughes ${ }^{1}$, R.F. Averill ${ }^{2}$, N.I. Goldfield ${ }^{2}$; ${ }^{1}$ Yale University School of Medicine, New Haven, CT; ${ }^{2} \mathrm{M}$ Health Information Systems, Wallingford, CT (Tracking ID \#75921)

BACKGROUND: Hospital discharge data that distinguish diagnoses present and not present on admission, now mandated by several states, make it possible to assess a patient's severity of illness (SOI) on admission, and to identify potentially preventable complications (PPCs) that arise after admission. We hypothesized that the risk of PPCs would vary by the reason for admission, and increase with increasing levels of SOI on admission.

METHODS: Using the All-Patient Refined-Diagnosis Related Groups (APR-DRG) patient classification system, we created a Potentially Preventable Complications Module (PPCM) that assigned PPCs (diagnoses that arose after admission) into 1 of 67 complication categories (COMPCATS). We used diagnoses present on admission for 2 purposes: 1) to assign patients' reason for hospitalization to 1 of 62 risk pools (32 medical, 30 surgical), and 2) to assign patients to 1 of 4 APR-DRG SOI levels within each risk pool. We then applied the PPCM to California data for all hospital admissions except neonates for 1999 and 2000.

RESULTS: We present the results for selected COMPCATs for the three risk pools for Gastrointestinal (GI) surgery. The COMPCAT for pneumonia occurred post-operatively in $2.9 \%$ of all GI surgery cases, but increased from $0.9 \%$ for the Minor GI surgery risk pool, to $3.3 \%$ for Moderate GI surgery, to $6.3 \%$ for Major GI surgery. Within each risk pool, risk increased with increasing SOI level (see table).

\section{Risk of Post-Op Pneumonia (\%):} Risk Pool

Minor GI Surgery

Moderate GI Surgery

Total GI Surgery
Major GI Surgery

\begin{tabular}{cccc} 
& \multicolumn{2}{c}{ SOI Level at } & Admission \\
$\mathbf{1}$ & $\mathbf{2}$ & $\mathbf{3}$ & $\mathbf{4}$ \\
0.3 & 1.4 & 4.2 & 11.4 \\
1.0 & 3.5 & 9.9 & 14.8 \\
1.7 & 4.6 & 13.0 & 19.6 \\
0.7 & 2.8 & 10.5 & 18.5
\end{tabular}

Total

0.9

3.3

6.3
We found similar gradients for all other COMPCATs within GI Surgery, both by risk pool and SOI level. For example, post-operative wound infection, which occurred in $1.6 \%$ of all GI surgery cases, increased from $0.3 \%$ for SOI level 1 in Minor GI, to $5.9 \%$ for SOI level 4 in Major GI surgery. Furthermore, the risk of PPCs increased by SOI level for all but 2 of 67 COMPCATs in all 62 risk pools.

CONCLUSION: Because the risk of potentially preventable complications varies by the reason for admission, and increases with increasing SOI on admission, the PPCM can improve the accuracy of screening for in-hospital complications.

PATIENT SATISFACTION IN A PRIMARY CARE CLINIC, A STRUCTURAL EQUATION MODEL. J.L. Jackson ${ }^{1}$; ${ }^{1}$ Uniformed Services University of the Health Sciences, Bethesda, MD (Tracking ID \#75719)

BACKGROUND: Traditional modeling methods are limited to assessing direct relationships between independent and dependent variables. Using such methods, we previously published a model of satisfaction that explained $20 \%$ of the variance: Satisfaction $=$ Age + Patient expectations + Patient functioning + Patient-doctor interaction (receiving diagnostic and prognostic information). Our purpose was reassess this model using structural equation modeling (SEM), a novel method that allows exploration of more complex interrelationships between variables.

METHODS: 500 adults presenting with physical symptoms to a primary care clinic were surveyed. Patient variables included symptom characteristics, worry, stress, mental disorders (PRIME-MD), expectations, functional status (SF-6) and satisfaction (Rand-9). Doctor variables included encounter difficulty (DDPRQ) and psychosocial orientation.

RESULTS: The resulting, well fitted SEM model explained $35 \%$ of the variance and suggested that satisfaction is explained by a different set of variable interactions:

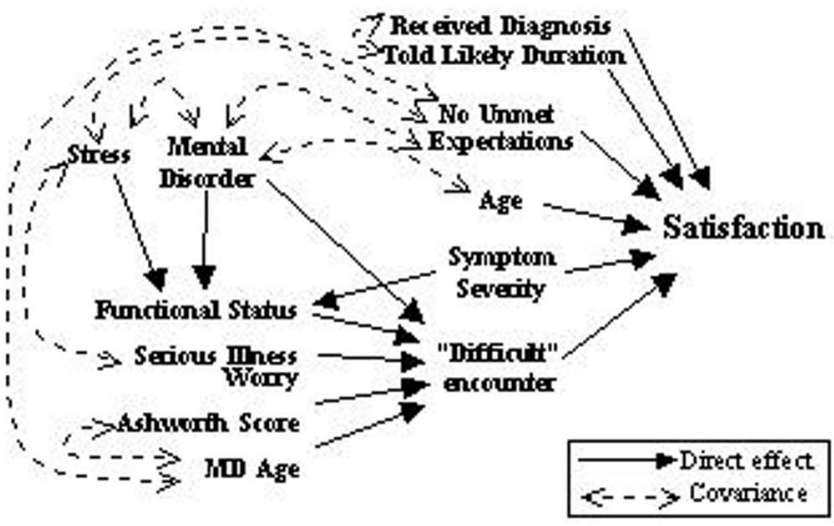

CONCLUSION: SEM allows a better model of the intricate web of interactions that comprise satisfaction. Traditional regression models may be misleading.

THE IMPACT OF PERFORMANCE MEASUREMENT ON VACCINATION RATES AND PNEUMONIA ADMISSIONS IN THE VETERANS AFFAIRS HEALTHCARE SYSTEM. A.K. Jha ${ }^{1}$, S.M. Wright ${ }^{2}$, J.B. Perlin ${ }^{2}$; ${ }^{1}$ Brigham and Women's Hospital, Boston, MA; ${ }^{2}$ Veterans Health Administration, Washington, DC (Tracking ID \#73623)

BACKGROUND: Influenza and pneumococcal vaccines are highly effective at reducing morbidity and mortality among the elderly and immunocompromised. Yet, they are widely underused. VA instituted the Performance Measurement Program (PMP) to improve quality of care, yet its impact on the rates of vaccinations and admissions due to pneumonia is not widely known.

METHODS: The study employed a cross-sectional design which sampled veterans who visited VA clinics in 1995 and annually between 1997 and 2000. Initiated in 1996, the PMP holds clinicians and managers accountable for achieving prespecified performance levels. The outcome variables were the rates of influenza and pneumococcal vaccination among eligible patients before and after the institution of PMP. The clinical impact of changes in vaccination were assessed by measuring rates of admissions due to pneumonia among the elderly in VA and Medicare between 1995 and 2000.

RESULTS: Within 4 years of the institution of the PMP, national rates of influenza and pneumococcal vaccination rose from a baseline of $27 \%$ and $26 \%$ to $77 \%$ and $80 \%$ respectively. Outside VA, influenza vaccination rates rose from $60 \%$ to $67 \%$ pneumococcal vaccination rates rose from $38 \%$ to $55 \%$. VA pneumonia admission rates were stable prior to the institution of the PMP, but after its adoption, the annual rate of hospitalization due to community acquired pneumonia among the elderly fell from 10.3 per 1000 enrollees in 1995 to 5.4 per 1000 enrollees in the year 2001. Over the same period, Medicare rates of pneumonia admissions rose by nearly $15 \%$.

CONCLUSION: VA experienced significant improvements in vaccination and large reductions in pneumonia admissions, most likely due to the institution of the performance program. Improvements in vaccinations far outstripped those outside VA and while national pneumonia rates were rising, VA's rates of pneumonia admissions were halved. Specification of goals, periodic feedback, and accountability for performance encompassed in VA's PMP may serve as a model for large healthcare systems seeking to improve preventive care and clinical outcomes.

DECLINING RATES OF AUTOPSY IN NORTH CAROLINA 1968-2000. J.D. Joines ${ }^{1}$ ${ }^{1}$ University of North Carolina at Chapel Hill and the Internal Medicine Program, Moses Cone Hospital, Greensboro, NC (Tracking ID \#76419)

BACKGROUND: Despite evidence supporting the importance of autopsy in establishing cause of death, autopsy rates have declined nationally for several decades. The goal of this study was to quantify the decline in autopsy rates in North Carolina since 1968, and to identify predictors of the autopsy decision.

METHODS: Autopsy rates were examined using anonymous statewide mortality file data for North Carolina decedents age 18 years and over for the years 1968 through 2000. Multiple logistic regression analysis was used to model predictors of autopsy, including age, gender, race, place of death, marital status, death in a Metropolitan Statistical Area (MSA), death in the county of residence, year, and type of certifier. Adjusted odds ratios from this model are shown below.

RESULTS: The overall autopsy rate was $12.8 \%$ in 1968 , reached a maximum of $14.5 \%$ in 1975 , and subsequently declined to $5.9 \%$ in 2000 . Rates for decedents in general hospitals declined from $15.6 \%$ in 1968 to $6.1 \%$ in 2000 . University hospitals had higher rates than nonuniversity hospitals $(35.3 \%$ vs $14.5 \%$ in 1968$)$, but after 1974 these declined in a similar manner $(12.5 \%$ versus $5.1 \%$ in 2000$)$. Autopsy rates for decedents in rest/nursing homes were low and relatively constant, never exceeding $1 \%$ throughout the period studied. In the multiple logistic regression model, all predictors achieved statistical significance as a consequence of the large number of observations. Autopsy was less likely with increasing age; using age 18-24 as reference category, odds ratios were 1.58 for age $25-34,1.55$ for age $35-44,1.07$ for age $45-54,0.72$ for age $55-64,0.51$ for age $65-74,0.33$ for age $75-84$, and 0.18 for age $85 \&$ up. Compared to those who died at home, decedents in nursing homes/rest homes were less likely to undergo autopsy $(\mathrm{OR}=0.26)$, and those in general hospitals $(\mathrm{OR}=1.84)$ and other institutions $(\mathrm{OR}=3.32)$ were more likely. Certification by a medical examiner or coroner 
$(\mathrm{OR}=6.01)$, nonwhite race $(\mathrm{OR}=1.06)$, and divorced marital status (versus never married) $(\mathrm{OR}=1.07)$ were associated with a higher likelihood of autopsy. Death in an MSA $(\mathrm{OR}=0.69)$, residence in the county of death $(\mathrm{OR}=0.72)$, married $(\mathrm{OR}=0.90)$ or widowed $(\mathrm{OR}=0.84)$ marital status, female gender $(\mathrm{OR}=0.95)$, and year $(\mathrm{OR}=0.97$ for one-year increment) were associated with a lower likelihood of autopsy.

CONCLUSION: In parallel with national trends, autopsy rates in North Carolina have declined substantially over the past three decades. This important quality control measure is likely underused, especially for the elderly and decedents in nursing and rest homes.

AUTOPSY IN HIV DISEASE: DECLINING RATES IN NORTH CAROLINA 1987-2000. J. Joines ${ }^{1}$; ${ }^{1}$ University of North Carolina at Chapel Hill and the Internal Medicine Program, Moses Cone Hospital, Greensboro, NC (Tracking ID \#77047)

BACKGROUND: Autopsy rates in general have declined in recent years, despite evidence that valuable information is often found at autopsy when HIV disease is the underlying cause of death. In this study, autopsy rates and correlates of autopsy were examined for North Carolina adults who died of HIV disease during the period 1987 to 2000.

METHODS: Autopsy rates were examined using anonymous statewide mortality file data for NC decedents age 18 years and older who died of HIV disease between 1987 and 2000. Multiple logistic regression analysis was used to model predictors of autopsy including age, sex, race, marital status, place of death, death in a Metropolitan Statistical Area (MSA), death in the county of residence, year of death, and type of certifier. Adjusted odds ratios from this model are shown below.

RESULTS: Autopsy rates for those who died of HIV disease declined from $20.1 \%$ in 1987 to $2.8 \%$ in the year 2000. Corresponding rates for those who died of underlying causes other than HIV were initially lower at $9.4 \%$ in 1987 , but by 1991 exceeded the autopsy rate for those who died of HIV disease $(8.4 \%$ vs $8.1 \%$ ), and remained higher throughout the decade $(5.9 \%$ vs $2.8 \%$ in 2000). In the multiple logistic regression model, certification by a medical examiner or coroner was the strongest predictor of autopsy $(\mathrm{OR}=26.0)$. Compared to those who died at home, decedents in general hospitals $(\mathrm{OR}=3.79)$ and other institutions $(\mathrm{OR}=2.42)$ were more likely to undergo autopsy. Female gender was associated with a higher likelihood of autopsy $(\mathrm{OR}=1.37)$. Decedents age 45 years and older were less likely to undergo autopsy $(\mathrm{OR}=0.67)$ than those age 18 to 34 years. Death in an MSA $(O R=0.49)$, death in the county of residence $(\mathrm{OR}=0.41)$, and year $(\mathrm{OR}=0.85$ for one year increment $)$ were associated with a lower likelihood of autopsy.

CONCLUSION: Autopsy rates for those who died from HIV disease in North Carolina declined substantially from 1987 to 2000 . This raises the concern that potentially treatable conditions which contribute to mortality may increasingly go unrecognized in patients with HIV disease.

IDENTIFICATION OF ADVERSE DRUG EVENTS AND MEDICATION ERRORS: POOR AGREEMENT BETWEEN CHART REVIEW AND PHYSICIAN, NURSE, AND PATIENT REPORT. P. Kaboli ${ }^{1}$, C. Jaipaul ${ }^{1}$, W. Barry ${ }^{1}$, J. Bennett ${ }^{2}$, B. Mutnick ${ }^{2}$, E. Jones ${ }^{1}$, G. Rosenthal ${ }^{1}$; ${ }^{1}$ lowa City VAMC and University of lowa, lowa City, IA; ${ }^{2}$ University of lowa, lowa City, IA (Tracking ID \#76790)

BACKGROUND: The reported percentage of hospital patients who experience an adverse drug event (ADE) or medication error (ME) varies considerably. Differences between studies may reflect the lack of an acceptable gold standard for determining the rate of events. The purpose of our study was to compare rates of ADEs, potential ADEs (PADEs), and MEs using multiple reporting methods.

METHODS: We conducted a prospective cohort study of 133 consecutive admissions to general internal medicine ward over an 8-week period and evaluated patients for ADEs, PADEs, and MEs. Four reporting strategies were employed: physician (MD) report, nurse (RN) report, patient interview, and chart review. Three physicians and two pharmacists adjudicated all events to determine type and severity by consensus.

RESULTS: $54 \%$ of study patients were male with a mean age of 54 years and mean length of stay of 7.9 days. 106 events were identified in $60(45 \%)$ different patients, of which 37 were ADEs, 41 were PADEs, and 28 were MEs. Of the 106 events, $53 \%$ were identified by chart review only, $8 \%$ by RN report only, $9 \%$ by MD report only, and $11 \%$ by patient report only. Only $19 \%$ were identified by more than one method, and no events were identified by all four methods. Agreement was generally low between chart review and the three other reporting strategies, with only a $29 \%$ concordance with MD report, $26 \%$ with RN report, and $26 \%$ with patient report. Kappa values for the agreement between chart review and the other three methods were all less than zero ( -0.14 with $\mathrm{MD},-0.15$ with $\mathrm{RN},-0.18$ with patients). Of the $37 \mathrm{ADEs}$, only $6(16 \%)$ were due to a medication error and only $10(27 \%)$ were preventable. Of the five ADEs considered life threatening, all were preventable, and all were reported in the chart. However, only two were reported by RNs, two by MDs, and only 1 by a patient. Two involved narcotics, one an anticoagulant, one potassium, and one a hypoglycemic. In addition, only 8 of the 106 events were reported to the voluntary hospital reporting system.

CONCLUSION: These results demonstrate that agreement between individual methods of $\mathrm{ADE} / \mathrm{ME}$ reporting is very poor and that alternative methods may be complementary to help identify events. The findings have important implications for the development of ADE/ME surveillance systems, the design of interventions to decrease event rates, and future research protocols in medication related errors and adverse events.

LINKING STRUCTURE, PROCESS, AND OUTCOMES FOR HYPERTENSION. K.L. Kahn ${ }^{1}$, C.L. Damberg ${ }^{2}$, J.L. Adams ${ }^{3}$, W.P. Chen ${ }^{1}$, D.M. Tisnado ${ }^{1}$, C.M. Mangione ${ }^{1}$ H. Liu ${ }^{1} ;{ }^{1}$ UCLA, Los Angeles, CA; ${ }^{2}$ PBGH, San Francisco, CA; ${ }^{3}$ RAND, Santa Monica, CA (Tracking ID \#76811)

BACKGROUND: Donabedian's classic quality of care model motivates optimal structure and processes by assuming outcomes (OCs) are functions of process, which can be manipulated by variations in structure. The complex relationships between burden of illness, use, and process have rarely allowed researchers to elucidate a structure-process-OC relationship using observational data in the ambulatory setting. Analyses demonstrating improved OC (i.e., BP) as a function of structure and process (i.e., evaluation, monitoring, and treatment of hypertension (HYP) might motivate organizations (orgs) and providers to improve care. METHODS: We merged 1996 and 1998 patient (pt) self-report data $(\mathrm{n}=1028$ managed care pts) and clinically detailed, 30-month long medical (med) record data with surveys of med directors regarding structure of care. We defined the intensity of clinical structure by availability of nurses, paraprofessionals, and systems to assist with systematic evaluation of and intervention for BP, its risk factors, and complications. We defined process from 1996-8 as adherence to evidence based clinical measures pertinent to BP control using the med record as a data source. We studied the intensity of interventions aimed at optimizing exercise, weight, nutrition, medications, and follow-up. We defined the dependent variable as elevated mean of the last 3 BP values in 1998 (i.e, systolic $>140$ or diastolic $>90$ ). We used OLS and logistic regression to respectively model process, and then BP control adjusted with Huber methods for clustering of pts w/in med orgs. The prediction of process and BP were each adjusted for $\mathrm{pt}$ demographic and comorbid characteristics, receipt of care within a MG or IPA, and corporate structure to facilitate pt and provider management of BP. Process was included in the model predicting BP.

RESULTS: After adjustment for pt characteristics, more intense org structure trended toward better process $(\mathrm{p}=.06)$. After adjustment for pt characteristics and structure, better process is associated with better BP control $(\mathrm{p}<.005)$. Compared with pts with best process, the OR for progressively worse process (defined as quartiles) is $1.2,1.4$, and 1.5 . Results were confirmed using a propensity score model.

CONCLUSION: Efforts by orgs and providers to deliver interventions known to be associated with better BP control in randomized trials are associated with better BP control in a large 3state sample of managed care pts. Knowing that structure and better process are associated with improved pt OCs (i.e., better BP control), can motivate orgs to design structures, and providers to identify methods to optimize processes that ultimately could have a big effect on national rates of MI, stroke, and renal failure.

THE IMPACT OF CONCURRENT DYSLIPIDEMIA AND DIABETES ON HYPERTENSION MANAGEMENT AND GOAL ATTAINMENT. D. Pettitt ${ }^{1}$, A.J. Karter ${ }^{2}$, T.Y. Peng ${ }^{2}$, A. Arnold ${ }^{1}$ J.V. Selby ${ }^{2} ;{ }^{1}$ Pfizer Outcomes Research, New York, NY; ${ }^{2}$ Kaiser Permanente of Northern California, Oakland, CA (Tracking ID \#76932)

BACKGROUND: Hypertension (HT), dyslipidemia (DL), and diabetes (DM) are strong predictors of cardiovascular disease, and often co-occur. We examined HT management and subsequent systolic blood pressure (SBP) goal attainment in individuals with concurrent HT, DL and DM.

METHODS: We measured SBP goal attainment and the number of antihypertensive therapy (AHT) classes used in the 2.1 million members, age $20 \mathrm{yrs}+$, of Kaiser Permanente Northern California during the 18 months prior to Dec. 31, 2001. Disease was defined using diagnosis codes, current treatment, or elevated blood pressure or lab values, using JNC-VI and ATP-III risk-based cutoffs. DM was identified from the health plan's DM registry (sensitivity $>98 \%$ ). RESULTS: Thirty-five percent of non-diabetic HT/DL patients were at SBP goal, compared with $25 \%$ of those with DM,although this is likely partially attributable to lower JNC-VI cutpoints applied to diabetic patients. HTN patients with either DL or DM were more likely to receive AHT therapy and utilize multiple medication groups.

CONCLUSION: Hypertensive patients with comorbid DL and/or DM are more likely to receive therapy and multiple classes of drugs than patients with HT alone. Despite this, goal attainment is poor for patients with concurrent DL or DM.

\section{Goal attainment and utilization of hypertensive medication}

\begin{tabular}{|c|c|c|c|c|c|c|c|}
\hline $\begin{array}{l}\text { Diabetes } \\
\text { Status }\end{array}$ & $\begin{array}{l}\text { HTN/DL } \\
\text { status }\end{array}$ & $\begin{array}{l}\text { Sample } \\
\text { Size }\end{array}$ & $\begin{array}{c}\text { At } \\
\text { SBP } \\
\text { Goal* }^{*} \\
(\%)\end{array}$ & $\begin{array}{c}\text { No } \\
\text { AHT } \\
\text { Therapy } \\
\text { (\%) }\end{array}$ & $\begin{array}{l}1 \text { AHT } \\
\text { Therapy } \\
\text { Class } \\
(\%)\end{array}$ & $\begin{array}{c}2 \text { AHT } \\
\text { Therapy } \\
\text { Classes } \\
(\%)\end{array}$ & $\begin{array}{c}3+\mathrm{AHT} \\
\text { Therapy } \\
\text { Classes } \\
(\%)\end{array}$ \\
\hline $\begin{array}{l}\text { No } \\
\text { Diabetes }\end{array}$ & $\begin{array}{l}\text { HTN } \\
\text { Alone }\end{array}$ & 228,844 & $36 \%$ & $46 \%$ & $32 \%$ & $16 \%$ & $6 \%$ \\
\hline $\begin{array}{l}\text { No } \\
\text { Diabetes }\end{array}$ & $\begin{array}{c}\mathrm{HTN}+ \\
\text { DL }\end{array}$ & 147,501 & $35 \%$ & $28 \%$ & $37 \%$ & $24 \%$ & $11 \%$ \\
\hline Diabetes & $\begin{array}{l}\text { HTN } \\
\text { Alone }\end{array}$ & 23,077 & $24 \%$ & $30 \%$ & $33 \%$ & $23 \%$ & $14 \%$ \\
\hline Diabetes & HTN + & 73,764 & $25 \%$ & $21 \%$ & $31 \%$ & $26 \%$ & $22 \%$ \\
\hline
\end{tabular}

SPECIALTY OF ADMITTING PHYSICIAN AND OUTCOMES OF CARE IN HOSPITALIZED PATIENTS WITH POSSIBLE ACI. D.A. Katz ${ }^{1}$, T. Aufderheide ${ }^{2}$, M. Bogner ${ }^{1}$, P.R. Rahko ${ }^{1}$, H.P. Selker ${ }^{3}$; ${ }^{1}$ University of Wisconsin-Madison, Madison, WI; ${ }^{2}$ Medical College of Wisconsin, Milwaukee, WI; ${ }^{3}$ Tufts-New New England Medical Center, Boston, MA (Tracking ID \#77044)

BACKGROUND: Recent studies have demonstrated that elderly patients with acute myocardial infarction who receive outpatient management by a cardiologist have lower mortality and are more likely to receive recommended treatment, compared to similar patients who receive outpatient generalist care. The aim of this study is to determine whether patients admitted with possible acute cardiac ischemia (ACI) show differences in treatment and outcome across specialty of admitting physician.

METHODS: As part of a before-after trial of the Agency for Healthcare Research and Quality (AHRQ) Unstable Angina guideline, we enrolled 273 consecutive emergency department patients (ED pts) who were admitted with symptoms of ACI to a University hospital. Pts' ED evaluation included 12-lead ECG and pertinent history. Adverse events, in-hospital treatment, and follow-up care were assessed by 30 -day telephone interview and chart review. We used a 
modified version of the SF-20 to assess functional status. Using multiple regression, we analyzed treatment and 30-day functional status after adjustment for the estimated propensity of being admitted to a cardiology service, AHRQ guideline risk group, and predicted probability of ACI (with correction for clustering by admitting physician).

RESULTS: On average, physical functioning was higher in cardiology pts (adjusted difference $=5.5$ on a scale of $0-100,95 \%$ CI $0-11.1, \mathrm{p}=0.05$ ). There were no differences in role function, general health perception, social function, or mental health measures. Inhospital complications and deaths (combined) were uncommon in both groups (5 vs. $2 \%$ in cardiology vs. generalist pts, $\mathrm{p}=0.15$ ). Cardiology pts were more likely than generalist pts to receive aspirin (OR 4.1, 95\% CI 2.0-8.3), beta-blockers (OR 1.8, 95\% CI 1.1-2.9), and cardiac catheterization (OR 2.7, 95\% 1.0-7.5).

CONCLUSION: Our results suggest that physical function in pts who are admitted to a cardiology service is superior to that of pts admitted to a generalist service for evaluation of possible ACI (at 30-day follow-up). One possible explanation is that cardiology pts are more likely to receive recommended therapies for ACI. Analysis of a larger sample of patients is needed to confirm these findings and to determine whether cardiology admission is associated with improved (or worsened) mortality and cardiac complications.

THE COST OF COMPLICATIONS: PNEUMONIA AFTER ACUTE STROKE. I.L. Katzan ${ }^{1}$, R.D. Cebul ${ }^{2}$; ${ }^{1}$ Case Western Reserve University, Cleveland, $\mathrm{OH} ;{ }^{2}$ Case Western Reserve University, Hunting Valley, $\mathrm{OH}$ (Tracking ID \#76800)

BACKGROUND: There is evidence that clinical pathways that include a swallowing evaluation can reduce the incidence of pneumonia that complicates acute stroke, although, in usual care, fewer than half of stroke patients are evaluated. We previously reported that pneumonia after stroke confers a 3 -fold increased risk for 30-day mortality; in this study, we examine the incremental costs of pneumonia complicating acute stroke.

METHODS: This was a retrospective cohort study involving 11,286 Medicare patients admitted for ischemic or hemorrhagic stroke to one of 30 hospitals in Greater Cleveland between 1991 and 1997. The sample excludes 3,007 patients who died or had DNR orders within 2 days of admission. For costs, we used patient-level charges adjusted for hospitalspecific cost-to-charge ratios, which then were log-transformed. Pneumonia was identified from secondary ICD-9-CM codes. We used detailed chart-abstracted patient data to generate covariates representing patient severity $(\mathrm{c}$-statistic $=.73)$, predicted cost $(\mathrm{r} 2=.37)$, and propensity for pneumonia $(\mathrm{c}$-statistic $=.83$ ). Linear regression was used to estimate the incremental cost of pneumonia after adjusting for age, sex, race, and the above covariates. RESULTS: Pneumonia occurred in 5.6\% (635/11286) of patients in the cohort. There was a higher incidence in patients with hemorrhagic stroke $(13.3 \%$ vs $4.9 \%, \mathrm{p}<.001)$, those admitted from nursing homes $(10.8 \%$ vs $5.3 \%, \mathrm{p}=.001)$, and those with greater severity on admission (predicted mortality $13.2 \%$ vs $6.0 \%, \mathrm{p}<0.001$ ). The unadjusted average cost for patients with pneumonia was $\$ 15,026$, compared to $\$ 5,094$ for patients without pneumonia, resulting in an incremental in-hospital cost of $\$ 9,932$. After adjustment, the cost for the average patient with pneumonia was $\$ 9,259$ as compared to $\$ 5,642$ for those without pneumonia, representing an incremental cost of $\$ 3,618$.

CONCLUSION: Extrapolated to the estimated 553,000 U.S. patients annually suffering acute stroke, the cost of pneumonia complicating stroke admissions is approximately $\$ 112$ million each year. Together with the 3 -fold increased mortality risk of pneumonia, and evidence that interventions can reduce risk, these findings provide strong impetus to quality improvement efforts in stroke evaluation and management.

BUILDING A BETTER QUALITY MEASURE: ARE SOME PATIENTS WITH "POOR QUALITY"' ACTUALLY GETTING GOOD CARE? E.A. Kerr ${ }^{1}$, D.M. Smith ${ }^{1}$, M.M. Hogan², T.P. Hofer ${ }^{1}$, S.L. Krein ${ }^{1}$, M. Bermann ${ }^{3}$, R.A. Hayward ${ }^{1} ;{ }^{1}$ Ann Arbor VAMC Center for Practice Management and Outcomes Research and University of Michigan Department of Medicine, Ann Arbor, Ml; ${ }^{2}$ Ann Arbor VAMC Center for Practice Management and Outcomes Research, Ann Arbor, MI; ${ }^{3}$ John D. Dingell VA Medical Center and the Department of Internal Medicine, Wayne State University, Detroit, MI (Tracking ID \#74891)

BACKGROUND: National performance measures monitor the proportion of diabetic patients with low density lipoprotein cholesterol (LDL) levels $\geq 130 \mathrm{mg} / \mathrm{dl}$, but such simple rates of substandard intermediate outcomes measure poor control, not poor care. Electronic medical records may enhance our ability to improve quality assessment by allowing the creation of more "tightly linked" quality measures that define good quality either by a good intermediate outcome $(\mathrm{LDL}<130 \mathrm{mg} / \mathrm{dl}$ ) or by evidence of appropriate responses to poor control (e.g., starting or optimizing medications for high LDL or not doing so in the face of contraindications).We examined hyperlipidemia therapy for patients with diabetes to determine the relative accuracy of quality assessment using simple intermediate outcome versus tightly linked quality measures.

METHODS: We used a national VA diabetes registry to randomly sample 1154 diabetic patients from two large VA healthcare systems who had an LDL test done between October 1, 1998-March 31, 1999. We reviewed the medical records of all patients with high LDL levels to more fully examine medication treatment for hyperlipidemia, contraindications to treatment and explanations for poor quality.

RESULTS: While $27 \%(307 / 1154)$ of patients had an LDL $\geq 130 \mathrm{mg} / \mathrm{dl}$ using the simple intermediate outcome measure, only $13 \%(148 / 1154)$ were classified as having substandard quality using the tightly linked measure. Among the 159 reclassified to adequate quality, 117 had lipid lowering medication started or increased within 6 months of an LDL $\geq 130 \mathrm{mg} / \mathrm{dl}, 8$ were already on high dose medication, 12 had a repeat LDL $<130 \mathrm{mg} / \mathrm{dl}$ and 22 had contraindications to treatment. We were able to construct a tightly linked measure from automated data alone that had a high agreement with the tightly linked measure constructed with medical record data $(\mathrm{Kappa}=0.84)$

CONCLUSION: Simple intermediate outcome measures can be an inaccurate reflection of true quality of care and many patients classified as having substandard quality by "poor control" may actually be receiving good quality of care.
DISCIPLINARY ACTION AGAINST PHYSICIANS: CHARACTERISTICS AND PREDICTORS. A.A. Khaliq ${ }^{1}$, L. Narine ${ }^{2}$, R.A. Smego ${ }^{3}$; ${ }^{1}$ University of Oklahoma Health Sciences Center, Oklahoma City, OK; ${ }^{2}$ University of North Carolina at Charlotte, Charlotte, NC; ${ }^{3}$ University of North Dakota School of Medicine \& Health Sciences, Fargo, ND (Tracking ID \#76109)

BACKGROUND: State medical boards routinely discipline physicians (MDs) for violations of laws governing the practice of medicine, but there is a dearth of information linking offender characteristics to severity of disciplinary action. We sought to determine physician characteristics and disciplinary predictors and to report the type, frequency, and severity of disciplinary actions against MDs.

METHODS: We undertook a descriptive and predictive analysis of publicly-available data maintained by the Oklahoma State Board for Medical Licensure and Supervision (OSBMLS) on disciplined MDs.

RESULTS: Longitudinally maintained data since 1922 showed that of 14,314 currently or previously licensed MDs, 396 (2.8\%) MDs had been disciplined. Forty-eight MDs currently had a revoked license, 19 were under suspension, and 79 were on probation. Of the 189 disciplined MDs currently practicing medicine, $85.7 \%$ were practicing in Oklahoma. While physician race was not a significant factor $(\mathrm{OR}=1.23,95 \% \mathrm{CI}=0.94-1.62)$, males $(\mathrm{OR}=2.62$, $95 \% \mathrm{CI} 1.79-3.84)$ and non-board-certified $\mathrm{MDs}(\mathrm{OR}=2.5,95 \% \mathrm{CI}=2.05-3.08)$ were more often disciplined. Logistic regression analysis showed that age $>40$ years, male gender, and lack of board certification were the only important predictors of being disciplined. During 2001, complaints against MDs involved quality of care issues (24.8\%), incompetence (18.3\%), malpractice $(16.7 \%)$, non-controlled substance fraud $(12.7 \%)$, billing issues $(9.4 \%)$, inadequate records $(8.4 \%)$, fraud $(6.7 \%)$, prescribing violations $(5.9 \%)$, sexual misconduct $(4.6 \%)$, substance abuse (3.8\%), criminal activity (3.0\%), fraud application $(0.3 \%)$, and others $(31.8 \%)$. For that same year, two-thirds of complainants came from a public source.

CONCLUSION: Since 1922, almost 3\% of its licensed MDs have been disciplined by the OSBMLS. The characteristics of offender MDs are similar to those previously reported.

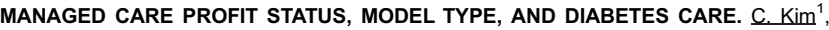
D.F. Williamson ${ }^{2}$, C.M. Mangione ${ }^{3}$, M.M. Safford ${ }^{4}$, J.V. Selby ${ }^{5}$, D.G. Marrero ${ }^{6}$, J.D. Curb $^{7}$, T.J. Thompson ${ }^{2}$, W.H. Herman ${ }^{1}$; ${ }^{1}$ University of Michigan, Ann Arbor, Ml; ${ }^{2}$ Centers for Disease Control and Prevention, Atlanta, GA; ${ }^{3}$ University of California, Los Angeles, Los Angeles, CA; ${ }^{4}$ University of Medicine and Dentistry of New Jersey, Livingston, NJ; ${ }^{5}$ Kaiser Permanente Division of Research, Oakland, CA; ${ }^{6}$ Indiana University Purdue University Indianapolis, Indianapolis, IN; ${ }^{7}$ University of Hawaii, Honolulu, HI (Tracking ID \#74030)

BACKGROUND: The influence of investor-ownership and managed care model on the quality of health care is controversial.

METHODS: We examined the relationship between health plan profit status, provider group model (group/network, group/staff, and independent practice association (IPA)/network), and diabetes quality of care measures: retinal, renal, foot, lipid, and hemoglobin A1C testing: aspirin advice; influenza vaccination; and a sum of these measures using data from the Translating Research into Action for Diabetes (TRIAD) Study. In TRIAD, surveys of managed care enrollees with diabetes $(n=11,921)$, directors of provider groups $(n=64)$ and plans $(n=10)$, and medical records were linked. Models adjusted for clustering at the plan and provider group levels, and patient age, gender, race, diabetes treatment, education, income, health status, and survey language.

RESULTS: Non-profit status was associated with slight but non-significant greater performance of quality of care measures (Table). Among for-profit plans, group/network models provided significantly more $(7-17 \%)$ of the measures of quality care than IPA/network models $(\mathrm{p}<0.01)$ except for aspirin advice. Among non-profit plans, little difference existed between provider group model types.

CONCLUSION: Profit status was not associated with greater performance of diabetes processes of care measures. Among for-profit plans, group/network models provide better diabetes care than IPA/network models.

Percentage of participants receiving diabetes processes of care and $(95 \% \mathrm{Cls})$

$\begin{array}{lccc} & \text { Non-profit } & \text { For-profit } & \text { Difference } \\ \text { Dilated eye exam } & 79(74-83) & 76(72-80) & 2 \\ \text { Urine protein checked } & 86(78-91) & 77(67-84) & 9 \\ \text { Foot exam performed } & 85(77-90) & 83(75-89) & 2 \\ \text { Lipids checked } & 63(51-73) & 67(57-76) & -4 \\ \text { HgbA1c checked } & 87(83-90) & 86(83-89) & 1 \\ \text { Aspirin advised } & 62(55-69) & 47(40-54) & 15 \\ \text { Flu vaccination } & 66(60-72) & 64(59-69) & 2\end{array}$

LINKING DIABETES PROCESSES TO OUTCOMES: THREE PARADOXES. S. Kim ${ }^{1}$, M.F. Wisniewski ${ }^{1}$, J. Bult ${ }^{1}$, L.A. Fogelfeld ${ }^{2}$, G.D. Schiff ${ }^{1} ;{ }^{1}$ Cook County Hospital, Chicago, IL; ${ }^{2}$ University of Illinois at Chicago, Chicago, IL (Tracking ID \#76136)

BACKGROUND: As quality assessment and health services research move from administrative to clinical databases, new vistas are opened for evaluating and linking care processes and outcomes. Clinical care for patients with diabetes mellitus affords such an opportunity as electronic data from pharmacy and laboratory become more widely accessible and linkable. In the course of a cross-sectional look at diabetes care in a large public hospital outpatient system we uncovered and sought to better understand 3 ?paradoxes? in relationships between outcomes and care processes.

METHODS: We downloaded all insulin and oral hypoglycemic agent prescriptions for 2001 from an NDC outpatient pharmacy database for a public hospital clinic system. Using ?unit number? identifier, unique patients were identified, and linked to all $\mathrm{Hgb} \mathrm{AlC}$ and glucose tests recorded in a laboratory database. Self-monitoring strip prescriptions dispensed (recorded in pharmacy database) were also downloaded and linked using Microsoft Access and SPSS. 
RESULTS: Overall 13,341 (66.3\%) patients were treated with oral agents, 3,767 (28.2\%) with insulin, 3,006 (22.5\%) with both. Recorded $\mathrm{Hgb} \mathrm{A1C}$ and glucose levels varied significantly with intensity of treatment with, paradoxically more intensive treatment associated with worse control. Glucose self monitoring strips were used by $59.5 \%$ in oral $74.8 \%$ with insulin, and $85.7 \%$ with both. There were no significant differences in glucose and $\mathrm{A} 1 \mathrm{C}$ control for insulin-using patients. For patients on oral agents (for whom self testing is less strongly recommended) there was a significant difference in recorded glucose levels when strips were being used $(183.3$ vs. $191.6, \mathrm{p}<.01)$ with A1C differences that fell slightly short of significant difference ( 8.56 vs. 8.64). Analysis of renal status revealed large numbers and \%s of patients with early and advanced renal insufficiency: $8863(41.8 \%)$ patients $\mathrm{Cr}<$ $1.19,2291(10.8 \%)$ Cr. $1.2-.99,429(2.0 \%)$ Cr 2.0-2.99, 145 (0.7\%) 3.0-3.99, and 328 $(1.5 \%) \mathrm{Cr}>4.0$. A third paradox was the finding that A1C varied inversely with worsening renal status (mean $\mathrm{A} 1 \mathrm{C}$ of 8.8 for $\mathrm{Cr}<1.19,8.8$ for 1.2 to $1.99,8.6$ for 2.0 to $2.99,8.4$ for 3.0 to 3.99 , and 8.0 for greater than 4.0 ).

CONCLUSION: While not widely reported, we noted three paradoxes evident in cross sectional data from a large cohort of public hospital diabetes patients. Plausible explanations and hypotheses for future testing, based on understanding of the clinical characteristics of the patients, nature of lab physiologic data and testing, and limitations of cross sectional data were developed by a multidisciplinary team. These postulated explanations include: difficulties related achieving tight glucose control in this population, selection biases in cohort assembly and patient selection, and insulin physiology in renal insufficiency. Those undertaking quality assesments using clinical databases need to take these factors into account to avoid bias in interpreation of the results.

WHETHER, VS. HOW MUCH? INHALED STEROID USE FOR PATIENTS RECEIVING BAGONIST SPRAYS. S. Kim ${ }^{1}$, G.D. Schiff ${ }^{1}$, J. Bult ${ }^{1} ;{ }^{1}$ Cook County Hospital, Chicago, IL (Tracking ID \#76925)

BACKGROUND: U.S. and international guidelines stress the need/role for anti-inflammatory inhaled steroid treatment for asthma patients regularly using beta agonists inhalers. Compliance with the recommendation has become a widely used quality indicator. In reviewing physician compliance with steroid prescriptions, we studied the extent to which compliance with this indicator (i.e. any steroid inhaler prescription in past year) reflected adequate/continuing protection with this beneficial therapy.

METHODS: We downloaded and linked electronic pharmacy records for all inhaled beta agonists and inhaled steroid prescriptions dispensed at a large public hospital outpatient pharmacy system for 2001. We downloaded three additional months of steroid inhaler prescriptions to conservatively ensure that patients first receiving beta agonists at the end of the calendar year would be captured. For each patient we analyzed whether any steroid inhaler had been dispensed, and for those receiving steroid inhalers we calculated how many months of "coverage" these steroid sprays represented as a function of the number of beta agonist canisters, using the steroid prescription sig of \# of puffs prescribed and spray quantity dispensed.

RESULTS: Overall 17,622 patients received beta-agonist inhaler therapy of whom 8,435 $(49.7 \%)$ also received a steroid at some time during the 12 month period; $9,095(51.6 \%)$ if a 15 month "capture-window" is used. \% of patients receiving any steroids varied by number of bagonist canisters dispensed during the 12 months: for 1 beta can-2499/8829 (28\%) received steroids, 3 beta cans 1244/1905 (65\%), 6 cans 449/518 (852\%), 9 cans-201/217 (93\%) 12 cans $70 / 72(97 \%)$. More detailed analysis (box plot) of the number of months patients actually "covered" with steroid inhalers varied widely.

CONCLUSION: Overall, the majority of patients dispensed b-agonist inhalers also received inhaled steroid prescriptions. However detailed calculation of what portion of the year patients were actually "covered" with inhaler steroid pharmaco-therapy reveals that many such patients received inadequate treatment, with many patients barely receiving a few months of inhaled steroid coverage, despite 6-12 or more canisters of beta agonist sparys used during that same year. Efforts to assess the quality of care for asthma patients should not be limited to simple categorical measures of whether steroid sprays were used, but rather should consider extent to which patient received adequate coverage throughout the year.

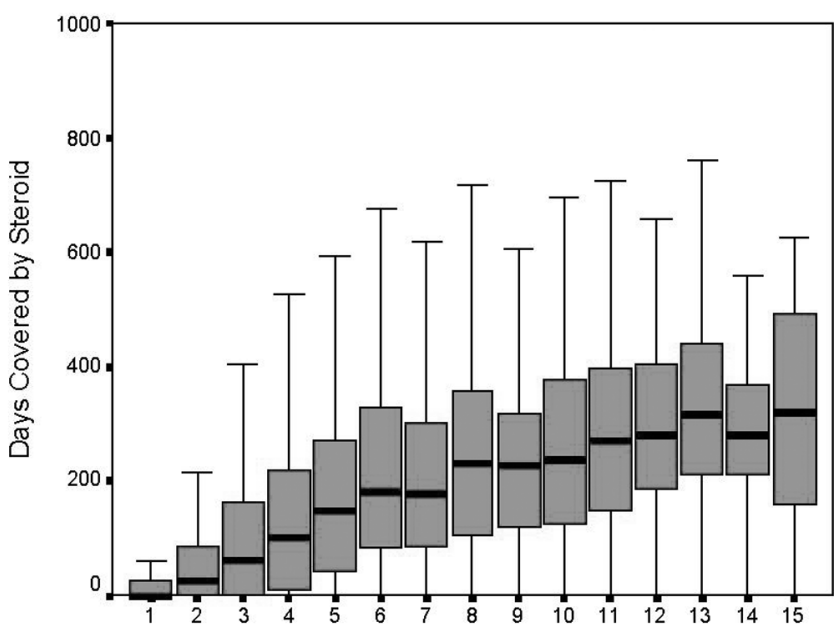

Number of Beta Cans Used
EVIDENCE-BASED MEDICINE, TREATMENT HETEROGENEITY, AND THE TYRANNY OF AVERAGE EFFECTS. R.L. Kravitz ${ }^{1}$, N. Duan ${ }^{2}$, J. Braslow ${ }^{2}$; ${ }^{1}$ University of California, Davis, Sacramento, CA; ${ }^{2}$ UCLA Department of Psychiatry, Los Angeles, CA (Tracking ID \#76210)

BACKGROUND: Evidence-based medicine (EBM) has won extraordinarily rapid acceptance by the scientific and clinical community. However, EBM has one major limitation: the problem of applying global evidence (mostly "average effects") to local needs (the sick individual as shaped by his genetic endowment and environment and cared for within a specific clinical setting). This problem is known to clinicians as "individualizing therapy" and to statisticians as "treatment heterogeneity."

METHODS: We reviewed the literature on the definition, sources, and implications of treatment heterogeneity $(\mathrm{TH})$ in clinical research and practice. We then considered how $\mathrm{TH}$ threatens the EBM paradigm and how the concepts might be reconciled.

RESULTS: Treatment heterogeneity derives from the observation that treatments which are mildly beneficial on average (compared to another treatment or no treatment) may be markedly beneficial for some patients, harmful in others, and ineffective in the rest. Yet randomized controlled trials and systematic reviews usually report average effects. Most trials are vastly underpowered for assessing treatments in specific subgroups. Treatment effects are heterogeneous because patients differ along four dimensions: 1) baseline risk of adverse outcomes; 2) responsiveness to treatment; 3) vulnerability to side effects of therapy; and 4) preferences or values. Incorporating these dimensions into medical decision making can enhance the likelihood that an individual will derive net benefit from treatment. The net benefit can be formulated as: (the utility-adjusted risk of disease- related adverse events times the reduction in relative risk due to treatment) minus (the utility-adjusted risk of treatmentrelated harm). The treatment threshold will be lower for patient subgroups with a higher relative risk of benefit (i.e., greater treatment responsiveness).

CONCLUSION: Average effects apply to average patients. By attending to notions of risk, responsiveness, vulnerability, and utility, researchers can design better studies and clinicians can make better use of the results. Randomized trials should place a renewed emphasis on subgroup analysis and be supplemented by observational studies to identify patient cohorts with different risks of disease-related events and post-marketing studies to document treatment-related adverse effects and their risk factors.

ARE VERTEBRAL COMPRESSION FRACTURES BEING ADEQUATELY TREATED IN THE ACADEMIC PRIMARY CARE CLINIC? P.J. Kroth ${ }^{1}$, C.J. McDonald ${ }^{2}$, M.D. Murray ${ }^{3}$; ${ }^{1}$ Regenstrief Institute for Health Care, Indianapolis, IN; ${ }^{2}$ Indiana University Purdue University Indianapolis, Indianapolis, IN; ${ }^{3}$ Purdue University, Indianapolis, IN (Tracking ID \#74936)

BACKGROUND: Vertebral compression fractures, even when asymptomatic, are associated with future deleterious clinical events such as additional vertebral fractures, hip fractures, depression, pulmonary problems, and increased mortality. Many of these problems may be prevented when patients receive appropriate treatment. The aim of this study was to ascertain the proportion of female patients with vertebral compression fractures who received treatment for osteoporosis at least once and delineate the medications prescribed.

METHODS: A retrospective electronic medical record database search was conducted on all patients in an academic internal medicine primary care clinic with documented vertebral compression fractures as identified by chest $\mathrm{x}$-ray reports. Patients were seen in the clinic on at least two occasions after the chest $x$-ray date, were female, and were 50 years of age or older. All chest x-ray reports that were performed from June 1, 1992 through May 9, 2002 were included. The database search identified all prescription orders sent to the pharmacy by physician order entry. Internal studies have shown that $95 \%$ of patients seen in the clinic use the pharmacy that is connected to the physician order entry system.

RESULTS: 114 patients were identified using the database search criteria. 57/114 (50\%) received at least one medication for osteoporosis at least one time. $45 / 114(39 \%)$ were prescribed estrogen or hormone replacement therapy, 13/114 (11\%) Alendronate, 6/114 (5\%) calcitonin injection, and 11/114 (10\%) calcitonin nasal spray. Only 1/114 (0.9\%) received calcium supplementation by prescription. No patients were found to have been prescribed raloxifene, etidronate, or risedronate.

CONCLUSION: 1) Only $50 \%$ of patients with vertebral compression fractures ever received at least one prescription for osteoporosis. These data only record an attempt at treatment at least one time. Therefore, the number of patients receiving appropriate medication on an ongoing basis is likely to be much lower due to long term adherence issues. 2) The most common medication prescribed for these patients was a form of estrogen or hormone replacement therapy. It is not known how many of these patients were placed on estrogen or hormones for reasons other than for a vertebral compression fracture. 3) Only one patient received any prescription for calcium supplementation. Adequate dietary calcium $(1200 \mathrm{mg} /$ day) is the recommendation for all patients by the National Osteoporosis Foundation.

THE EFFECT OF A QUALITY IMPROVEMENT COLLABORATIVE ON THE CARE OF PATIENTS WITH HIV INFECTION. B.E. Landon ${ }^{1}$, I. Wilson ${ }^{2}$, K. Mclnnes ${ }^{1}$, M.B. Landrum ${ }^{1}$, L. Hirshhorn ${ }^{3}$, P.V. Marsden ${ }^{4}$, D.H. Gustafson ${ }^{5}$, P.D. Cleary ${ }^{4} ;{ }^{1}$ Harvard Medical School, Boston, MA; ${ }^{2}$ New England Medical Center, Boston, MA; ${ }^{3}$ Dimock Community Heatlh Center, Boston, MA; ${ }^{4}$ Harvard University, Cambridge, MA; ${ }^{5}$ University of WisconsinMadison, Madison, WI (Tracking ID \#75261)

BACKGROUND: Quality improvement methods have been applied in numerous health care settings for a variety of conditions, but there is little systematic evidence supporting their usefulness.

METHODS: In 1999, HRSA required that all new clinics that received funding under Title III of the Ryan White Care Act participate in a quality improvement collaborative. Other currently funded clinics were also invited to participate. Collaborative clinics were matched to control clinics by location (urban/rural), region, size, and clinic type. We abstracted quality of care data on nine quality indicators from pre- and post-intervention samples of patients from each clinic. Comparisons of change over time for intervention and control clinics were 
examined using hierarchical models controlling for patient characteristics and clinic characteristics.

RESULTS: We analyzed data from 44 intervention and 25 matched control clinics. Overall, 9,986 patients were studied including 6,406 from the intervention clinics and 3,580 from the control clinics for an average of 72 patients per clinic per round. All clinical and sociodemographic characteristics of the intervention and control patients were similar $(\mathrm{p}>.05)$. We found no significant differences in changes in the quality of care between intervention and control clinics. The proportion of patients with a suppressed viral load increased by 7.1 percentage points from $32.8 \%$ to $40.6 \%$ in the intervention group as compared with 1.9 percentage points from $37.9 \%$ to $39.8 \%$ in the control group but this difference was not statistically significant $(\mathrm{p}=.13)$ after adjustment. Rates of HAART use for appropriate patients were high in both the pre and post-intervention periods $(\sim 80 \%)$ with a slight decrease in the second period. There was no difference between the intervention and control sites. There were also no significant differences in rates of appropriate screening tests and prophylaxis although the differences tended to favor the intervention group.

CONCLUSION: In this prospective matched trial of a quality improvement collaborative that included almost 10,000 patients, we found that the collaborative did not significantly impact the quality of care. Our findings suggest that further research is needed to improve methods of teaching and implementing quality improvement programs in order to achieve better results.

ONLINE DISCHARGE DOCUMENTATION IMPROVES MEDICATION SAFETY AND TIMELINESS OF COMMUNICATION. J. Lee ${ }^{1}$, G. Fulton ${ }^{1}$, R. Nichols ${ }^{1}$, A. Gettinger ${ }^{1}$; ${ }^{1}$ Dartmouth Hitchcock Medical Center, Lebanon, NH (Tracking ID \#74572)

BACKGROUND: The moment of discharge from an inpatient to an ambulatory setting is a potentially high-risk step in the coordination of care. Accurate and timely medication data is a crucial element of post-discharge ambulatory care. We designed an online tool to improve accuracy of medication data and speed transmissal of this information at time of discharge. METHODS: A novel discharge note type, the Interdisciplinary Discharge Form (IDF), which embedded ambulatory medication information, was created within the larger propietary electronic medical record at Dartmouth-Hitchcock Medical Center, a 330 bed tertiary care hospital. All adult discharges were considered in two periods: 1) June 1-30, 2001, when inpatient notes and online medication lists had been deployed, but the IDF template had not been formulated; and 2) December 1-31, 2001, when the integrative IDF had been fully deployed. The discharges were assessed for: 1) the presence, on the day of discharge, of updates to the medication list and 2) the presence, on the day of discharge or one day prior, of the filing of an IDF. The medication updates were compared by a test of proportions. In a subsequent 60 day trial, the IDF was converted to a full discharge summary for the surgical but not medical inpatient services. Time from discharge to completion of the discharge summary (either via traditional dictation or via direct entry) was compared by test of proportions.

RESULTS: In period 1, there were 1096 discharges and 305 (27.8\%) recorded updates to the medication list. In period 3, among 1,029 discharges, there were 549 (53.4\%) updates to the medication list. Between the pre and post intervention periods, there was an absolute increase in medication updates of $79 \%$, which also proved to be stastically significant $(\mathrm{p}<0.001)$. Among 551 discharge notes filed in the intervention period, $549(99.6 \%)$ included an updated medication list. Upon the transition of the IDF to a full summary, in the study period there were 1,595 total discharges with $985(61.8 \%)$ dictated summaries and $610(38.2 \%)$ summaries entered directly. Among the direct entry cohort the mean time to completion of the record was 51 hours $(95 \% \mathrm{CI}=40-62)$ post discharge while it was 163 hours $(95 \% \mathrm{CI}=152-174)$ post discharge in the dictated summary cohort ( $\mathrm{p}<.001)$. Among the direct entry cohort, $78.4 \%$ of records were complete prior to the actual hour of discharge compared to only $2.4 \%$ of records in the dictated cohort $(\mathrm{p}<.001)$.

CONCLUSION: This study suggests that an online discharge document incorporating an update to an ambulatory online medication list can improve rates of medication list accuracy. However, as a "Time series" analysis it may not take into account other fundamental process improvements that led to increased medication list updating. Further study is required to determine if increased ambulatory medication list accuracy at time of discharge was linked to fewer outpatient adverse drug reactions. This study also demonstrated that use of an online discharge summary can improve timeliness of discharge documentation as it allows house officers to complete the job of documenting the hospital course and discharge instructions in one step instead of two.

DESCRIBING MEDICAL ERROR RATES FOR CONGESTIVE HEART FAILURE PATIENTS AT AN ACADEMIC MEDICAL CENTER. L. Isabell ${ }^{1}$, S.J. Peterson ${ }^{2}$, W. Lee ${ }^{1}$; ${ }^{1} \mathrm{New}$ York Medical College, Valhalla, NY; ${ }^{2} \mathrm{New}$ York Medical College, New York, NY (Tracking ID \#76212)

BACKGROUND: Medical errors lead to an estimated 90,000 deaths per year in the United States. Estimating the prevalence rates of medical errors may be useful in identifying what types of errors most likely lead to adverse events. Using a quality improvement model, we estimate the prevalence rates of medical errors for patients with congestive heart failure at an academic teaching hospital.

METHODS: Retrospective chart review of patients admitted with a primary diagnosis of congestive heart failure at a tertiary care academic medical center over a one-month period. Patients were excluded if they had chronic or acute renal failure, sepsis, or were admitted to the intensive care unit during the hospital course. For the initial 24-hour admission period, planning errors occurred when a patient did not have baseline labs drawn, a baseline chest film, and when oxygen, intravenous nitrates, or loop diuretics were not administered to the patient. Intent errors occurred when interventions were done, but not charted or acted upon. For the hospital course, planning errors occurred when volume status or left ventricular function was not assessed, and when angiotensin converting enzyme inhibitors were not started. Intent errors during this period occurred when loop diuretic dosage was not adjusted per volume status, or chart documentation was not noted for diagnostic studies or explaining why ACE inhibitors were not started.
RESULTS: 28 patients over a 4 week period with a primary diagnosis of congestive heart failure were reviewed. Mean length of stay was 9 (s.d. 6.6) days, 15 men and 13 women. A total of 149 errors were noted (61 planning, 88 intent errors). For the initial 24-hour period the mean number of planning and intent errors was 1.39 and 1.29 respectively. Non-use of intravenous nitrates accounted for most of the planning errors. During the hospital course, the mean number of planning errors was 0.79 . Mean intent errors were 0.68 for adjusting medications and 1.18 for adjusting the dose of ACE inhibitors.

CONCLUSION: Medical errors among patients admitted with congestive heart failure appear to occur commonly. Most errors occur in the initial 24-hour admission period, and at the time of discharge, which reflect busy times during a patient's hospital course. Efforts should be made to identify those factors that can decrease the rates of errors in these time periods.

USE AND IMPACT OF PERIOPERATIVE BETA BLOCKADE AMONG PATIENTS UNDERGOING MAJOR NONCARDIAC SURGERY. P.K. Lindenauer ${ }^{1}$, D. Mamidi ${ }^{2}$, B. Gutierrez ${ }^{3}$, P. Pekow ${ }^{2}$, E.M. Benjamin ${ }^{1}$; ${ }^{1}$ Tufts University School of Medicine, Springfield, MA; ${ }^{2}$ University of Massachusetts Amherst, Amherst, MA; ${ }^{3}$ Premier Healthcare Informatics, Charlotte, NC (Tracking ID \#76733)

BACKGROUND: The perioperative administration of beta blockers to selected patients undergoing major noncardiac surgery has been shown to reduce cardiovascular complications and mortality in several small randomized controlled trials. Little is known about the current use or impact of perioperative beta blockade in routine clinical practice.

METHODS: We conducted a retrospective cohort study of all 782,969 patients aged 18 and older who underwent major noncardiac surgery in 2000 and 2001 at one of 329 hospitals participating in Premier Perspective, a comparative quality and utilization database. Using ICD-9-CM secondary diagnoses we calculated a Revised Cardiac Risk Index (RCRI) score for each patient, assigning 1 point for each of the following risk factors: high risk surgery, ischemic heart disease, cerebrovascular disease, chronic renal insufficiency and diabetes mellitus. We considered a patient to be an ideal candidate for perioperative beta blockade if they had an RCRI score of 1 or higher and if they had no secondary diagnoses suggestive of a contraindication to beta blockade. We examined the use of beta blockers among ideal candidates and compared the in-hospital mortality of ideal candidates who received a beta blocker on the day of admission with those who did not receive a beta blocker during the hospitalization

RESULTS: Of 782,969 patients undergoing major noncardiac surgery, 343,415 (44\%) appeared to be ideal candidates for perioperative beta blockade. $42,351(12 \%)$ ideal candidates were treated with a beta blocker on the day of admission and 106,707 (31\%) received a beta blocker at some point during their hospitalization. Compared to ideal candidates who did not receive treatment with a beta blocker, those treated on the day of admission had a significantly lower mortality across all RCRI strata (see Figure).

CONCLUSION: The perioperative administration of beta adrenergic blockers is associated with reduced in-hospital mortality among intermediate and high risk patients undergoing major noncardiac surgery. There are large opportunities to improve quality of care by increasing the use of beta blockers in this population.

\begin{tabular}{|c|c|c|c|c|}
\hline $\mathrm{RCRI}$ & $\mathrm{H}$ & Beta Blocker Use (N) & Mortality $\%$ & OR $(95 \% \mathrm{CI})$ \\
\hline 1 & 250,587 & $\begin{array}{l}\text { Ho hospital use }(185,722) \\
\text { Used on day of admission }(26,081)\end{array}$ & $\begin{array}{l}2.22 \\
1.96\end{array}$ & $\begin{array}{l}1.00 \\
.88(.80, .97)\end{array}$ \\
\hline 2 & 75,947 & $\begin{array}{l}\text { No hospital use }(43,114) \\
\text { Used on day of admission }(12,704)\end{array}$ & $\begin{array}{l}5.38 \\
2.69 \\
\end{array}$ & $\begin{array}{l}1.00 \\
.49(.43, .55)\end{array}$ \\
\hline 3 & 15,480 & $\begin{array}{l}\text { No hospital use }(7,288) \\
\text { Used on day of admission }(3,248)\end{array}$ & $\begin{array}{l}7.75 \\
4.56 \\
\end{array}$ & $\begin{array}{l}1.00 \\
.57(.47,68)\end{array}$ \\
\hline$\geq 4$ & 1,401 & $\begin{array}{l}\text { No hospital use ( } 589) \\
\text { Used on day of admission ( } 318 \text { ) }\end{array}$ & $\begin{array}{l}10.87 \\
5.97\end{array}$ & $\begin{array}{l}1.00 \\
.52(.31, .89)\end{array}$ \\
\hline
\end{tabular}

AN INTERVENTION THAT EDUCATES WRIST FRACTURE PATIENTS AND THEIR PHYSICIANS CAN INCREASE THE DETECTION AND TREATMENT OF OSTEOPOROSIS - RESULTS OF A CONTROLLED TRIAL. S.R. Majumdar ${ }^{1}$, B.H. Rowe', D. Folk ${ }^{1}$, J.A. Johnson ${ }^{1}$, B.H. Holroyd ${ }^{1}$, D.W. Morrish ${ }^{1}$, C.H. Harley ${ }^{1}$, W.P. Maksymowych ${ }^{1}$, I. Steiner ${ }^{1}$, B. Wirzba ${ }^{1}$, D.A. Hanley ${ }^{2}$, A.S. Russell ${ }^{1} ;{ }^{1}$ University of Alberta, Edmonton, Alberta; ${ }^{2}$ University of Calgary, Calgary, Alberta (Tracking ID \#75494)

BACKGROUND: Fragility fractures of the wrist are the most common symptomatic fracture related to osteoporosis. Recent studies have found that $80-90 \%$ of wrist fracture patients $>50$ years of age are neither investigated nor treated for osteoporosis in the year post-fracture. Our purpose was to use a quality improvement intervention to increase detection and treatment of osteoporosis in older patients with fractures of the wrist treated in the Emergency Department (ED).

METHODS: The intervention included case-finding in the ED; patient counseling (written materials and brief telephone calls); and primary care physician education (patient-specific single page guidelines for bone mineral density (BMD) testing and treatment options, endorsed by local "opinion leaders"). The intervention was compared to usual care in a prospective, quasi-randomized, double-blind trial. Patients $>50$ years with a wrist fracture who were discharged home were eligible; those receiving osteoporosis treatment were excluded. Main outcomes were BMD testing and osteoporosis treatment (with bisphosphonates, calcitonin, raloxifene, or HRT) within 6 months of fracture.

RESULTS: Based on intervention efficacy and safety, our "a priori" stopping rules dictated that the trial be stopped after the first interim analysis, when 80 patients had had final outcomes ascertained. To date, 222 consecutive patients have been screened and 112 patients enrolled. Of the first 80 patients, 43 were allocated to intervention and 37 to usual care control. 
Main reasons for exclusion were osteoporosis treatment $(n=65)$ and admission to hospital $(n=42)$. Patients had a mean age of 67 years (range 50-92) and $78 \%$ were female. There were no significant differences in characteristics between intervention and control patients. Using intention-to-treat analyses, $65 \%$ of intervention patients had undergone BMD testing compared with $19 \%$ of controls 6 months post-fracture (absolute increase $46 \%, p<0.001$ ) Furthermore, $40 \%$ of intervention patients were started on osteoporosis treatment compared with $11 \%$ of controls (absolute increase $29 \%, \mathrm{p}=0.004$ ). All patients were treated with bisphosphonates.

CONCLUSION: We found that, compared with usual care, an Emergency Department based intervention has the potential to increase the rate of detection and treatment of osteoporosis in older patients with wrist fractures.

THE IMPACT OF METHADONE INDUCTION AND STABILIZATION ON CARDIAC CONDUCTION IN OPIATE USERS. B. Martell ${ }^{1}$, J.H. Arnsten ${ }^{2}$, M.N. Gourevitch ${ }^{2} ;{ }^{1}$ Albert Einstein College of Medicine, Bronx, NY; ${ }^{2}$ Montefiore Medical Center, New York, NY (Tracking ID \#76448)

BACKGROUND: Opiate agonist therapy, specifically levacetylmethadol (LAAM) and high doses of methadone $(>300 \mathrm{mg} /$ day) has been associated with QT interval prolongation and torsade de pointes. We sought to determine whether induction on methadone is associated with prolongation of the QT interval in opiate users newly enrolled in a methadone maintenance treatment program in the Bronx, NY.

METHODS: A prospective cohort study was conducted of 169 consecutive new admissions to methadone maintenance treatment. All had been addicted to opiates for $>1$ year and were $>18$ years old. At baseline, prior to methadone induction, a standard 12-lead electrocardiogram (ECG), medical history, and physical exam were performed. During follow-up, patients were stabilized on a methadone dose ranging from $30-150 \mathrm{mg}$, and ECGs were performed at 2 and 6 months. QT intervals were measured using the algorithmic program provided with the MAC 1200 ECG machine. The QT interval was then corrected (QTc) for heart rate using Bazett's formula (QT/square root (RR)). The mean of the difference between baseline and follow-up QTc intervals was tested for statistical significance using the Wilcoxon signed rank test for non-normally distributed data. Separate analyses were performed for the entire cohort and for pre-determined subgroups.

RESULTS: Subjects were $66 \%$ male, $68 \%$ Hispanic, $28 \%$ African American, $5 \%$ white, and the mean age was 40 (19-60 range) years. $15 \%$ were HIV-infected, and $8 \%$ were on HAART. At baseline, $100 \%$ reported heroin use, $44 \%$ reported cocaine use, $8 \%$ reported nonprescription benzodiazepine (BZ) use, and $17 \%$ reported drinking more than 10 drinks per week. After 2 and 6 months of methadone treatment, we observed a mean QTc interval increase of 10.5 and 11.9 milliseconds $(\mathrm{ms})$, respectively $(\mathrm{p}<.0001$ at both follow-up points). At 6 months, the greatest QTc intervals were observed in women $(13.7 \mathrm{~ms}, \mathrm{p}<0.01)$, subjects taking $>110 \mathrm{mg}$ of methadone $(21.3 \mathrm{~ms}, \mathrm{p}<0.05)$, subjects who reported no cocaine use at baseline $(13.9 \mathrm{~ms}, \mathrm{p}<0.001)$, subjects who reported non-prescription BZ use at baseline ( 45.4 $\mathrm{ms}, \mathrm{p}=0.06)$, and subjects who reported more alcohol use at baseline $(13.5 \mathrm{~ms}, \mathrm{p}=0.3)$. No adverse cardiac events were observed.

CONCLUSION: Methadone induction and early stabilization was associated with modest QTc interval prolongation at two and six months follow-up. However, the clinical significance of this finding is unclear. None of the patients exhibited a interval increase of $\geq 40 \mathrm{~ms}$, the generally accepted threshold for clinical concern, and all follow-up QTc intervals were well below the interval (> 500 milliseconds) associated with torsades de pointes. Further research is needed to determine whether QTc interval changes in patients receiving methadone are associated with adverse cardiac outcomes.

HOUSESTAFF ATTITUDES ABOUT REPORTING MEDICAL ERRORS. S.M. Maviglia ${ }^{1}$ C.L. Roy ${ }^{1}$, T.K. Gandhi ${ }^{1}$; ${ }^{1}$ Brigham \& Women's Hospital, Boston, MA (Tracking ID \#76365)

BACKGROUND: It is estimated that 44,000 to 98,000 people die each year at least partially from iatrogenic injury, and that 1.3 million are injured (IOM, 1999). Most health care organizations rely on spontaneous reporting systems (incident reports) to detect errors and adverse events, but these detect only a minority of the events which actually occur (Cullen DJ, et al., Jt Comm J Qual Improv, 1995). Discussing errors and near misses on daily rounds in the hospital can increase reporting (Weingart SN, et al., JGIM, 2001). For a reporting system to be successful, there needs to be a culture where staff feels secure in reporting errors, errors are viewed as systems (not personal) failures, and leadership is committed to increasing patient safety.

METHODS: All medicine housestaff at Brigham \& Women's Hospital were invited by email to complete a confidential web-based survey about their reporting behavior and their perception of how the hospital handles adverse events.

RESULTS: We received 41 responses from 186 housestaff (22\%). Only $17 \%$ said they would definitely or probably report an erroneous order if they were able to correct the mistake before the order was enacted; less than $10 \%$ would have informed the attending, hospital risk management, or the patient. For an error resulting in extra labs or treatment, the reporting rate was $95 \%$; however, only $53 \%$ would report the same error committed by a colleague. For an error causing a patient's death, $90 \%$ believed they would be disciplined, $83 \%$ thought it would appear negatively in their evaluation, and $32 \%$ thought they might be fired. Only half thought that the hospital had a non-punitive error reporting system, that their ideas were actively sought and used by by the hospital's leadership, and that senior medical staff puts a high priority on the subject. Less than $20 \%$ thought the hospital provided enough information about detected errors, yet over $80 \%$ thought errors should be publicly discussed, including near misses that do not result in patient harm. Over $90 \%$ believed that most medical errors are due to problems with processes rather than people, and $95 \%$ expressed the desire to learn more about the subject. CONCLUSION: Housestaff will report serious events which result in patient harm, but are less willing to report near misses, or events which involve a colleague. Housestaff expressed concern that reporting a serious adverse event would have negative consequences on their career, including possible loss of job. While many of the housestaff surveyed understood that errors and injuries are best dealt with using systems-oriented approaches, many believed that the hospital leadership should engage them more actively in identifying systems improvements. Education about actively reporting errors and providing feedback about identified cases could help bridge this gap.

FAILURE TO HOSPITALIZE FOR ACUTE CARDIAC ISCHEMIA: DOES HOSPITAL TYPE MAKE A DIFFERENCE? $\underline{\text { C. Milch }}{ }^{1}$, r. ruthazer ${ }^{1}$, j. beshansky ${ }^{1}$, J. Griffith ${ }^{1}$, h. selker ${ }^{1} ;{ }^{1}$ TuftsNew England Medical Center, Boston, MA (Tracking ID \#76180)

BACKGROUND: Thousands of patients with acute cardiac ischemia (ACI) are erroneously discharged from emergency departments (ED) each year. We evaluated rates of failure to hospitalize patients with ACI between publicly and privately funded metropolitan teaching hospitals.

METHODS: Data were obtained from a large 6-state, 10-hospital study conducted in 1993 of patients presenting to EDs with symptoms suggestive of ACI. The participating sites were teaching hospitals located in metropolitan areas. We used univariate and multivariate logistic regression to determine the odds of discharge from public vs. private hospitals in the subgroup of patients with acute myocardial infarction (AMI) or severe and/or new onset unstable angina pectoris (UAP). Analyses were adjusted for patient demographic, clinical and hospital characteristics to account for possible confounders.

RESULTS: Five publicly and five privately funded hospitals participated in the study. Of the 1,856 ED patients with ACI, $66 \%$ presented to private hospital EDs. Public vs. private hospital EDs had a statistically greater percentage of patients who were female (46 vs. $38 \%$ ), non-white ( 50 vs. $11 \%$ ), or had Medicaid or no health insurance ( 48 vs. $15 \%$ ) ( $<<0.05$ for all comparisons). The percentage of patients with abnormal ECGs and proportions of ACI symptom severity were similar between the two hospital types. A total of 41 patients $(2.2 \%)$ with ACI were discharged home from the ED ( $2.1 \%$ of those with AMI and $2.3 \%$ of those with UAP). Among the private hospitals, $1.1 \%$ of patients with $\mathrm{ACI}(13$ of 1,215$)$ were discharged compared with $4.4 \%$ (28 of 641) from public hospitals. After adjustment for age, gender, and ethnic category (white vs. non-white), patients with ACI presenting to public hospital EDs were more likely to be discharged home compared with patients in private hospital EDs (odds ratio 3.9, 95\% CI $1.9,8.2$ ). Adjusting separately for 1 ) patient characteristics (type and duration of symptoms at ED presentation, disease severity, ECG abnormalities, type of health insurance, socioeconomic factors), and 2) institutional factors (cardiac unit bed availability, presence of an ED residency program, hospital size, ED volume) did not substantially change the odds ratio for discharge (range 3.3 [adjusted for socioeconomic factors] to 5.2 [adjusted for health insurance]). After adjustment for hospital type, ethnic category was no longer a significant predictor for discharge from the ED.

CONCLUSION: Failure to hospitalize ED patients with ACI is three to five-fold more likely in public teaching than private teaching hospitals, even after adjustment for age, gender, race, socioeconomic level, type of health insurance, and clinical and institutional factors.

UNDERUSE OF CARDIOPROTECTIVE MEDICATIONS IN IHD PATIENTS PRIOR TO ACUTE MYOCARDIAL INFARCTION. R. Miller ${ }^{1}$, Y. $\mathrm{Li}^{2}, \mathrm{H}$. Sun ${ }^{2}$, B. Kopjar ${ }^{3}$, A.E. Sales ${ }^{4}$, S. Pineros ${ }^{2}$, S.D. Finn ${ }^{4} ;{ }^{1}$ Puget Sound Health Care System, Seattle, WA; ${ }^{2}$ Health Services Research and Development Center of Excellence, VA Puget Sound Health Care System, Seattle, WA; ${ }^{3}$ Department of Health Services, University of Washington, Seattle, WA: ${ }^{4}$ University of Washington, Seattle, WA (Tracking ID \#74882)

BACKGROUND: Secondary prevention in ischemic heart disease (IHD) improves survival, reduces recurrent cardiac events, improves quality of life and is cost-effective. Many current organizational performance measures focus on implementing drug therapies following an acute myocardial infarction (AMI). We evaluated changes in drug therapy before and after AMI to determine whether some patients had indications for these drugs prior to their AMI and whether there was an improvement in indicated drug prescription after the AMI.

METHODS: We conducted a before-after study of patients $(n=231)$ with documented IHD (ICD-9-CM codes $410,411,412,414$ ) to compare receipt of cardioprotective drugs during the six months prior to admission for AMI and during the three months following discharge. Patients, identified through the Veterans Administration Ischemic Heart Disease Quality Enhancement Research Initiative (IHD QuERI), were admitted with a primary diagnosis of AMI between July 1, 2000 and June 30, 2001. Pharmacy and co-morbidity data were extracted from the VISN 20 Data Warehouse (CHIPS).

RESULTS: We observed significant improvements $(\mathrm{p}<.05)$ in the proportion of patients $(\mathrm{n}=231)$ receiving medications before and after hospitalization for AMI for each of three drug classes: from $50 \%$ to $68 \%$ for statins; from $53 \%$ to $82 \%$ for beta-blockers; and from $50 \%$ to $66 \%$ for ACEI/ARB. The magnitude of the improvement varied in subgroups of patients with different cardiac risk factors: hypertension (27\% statins, 34\% beta-blockers, $21 \%$ ACEI/ARB); diabetes (11\% statins, $31 \%$ beta-blockers, $12 \%$ ACE/ARB); LDL greater than or equal to $120 \mathrm{mg} / \mathrm{dl}$ (35\% statins, $44 \%$ beta-blockers, $21 \%$ ACEI/ARB); congestive heart failure ( $14 \%$ beta-blockers).

CONCLUSION: A significant number of patients with IHD who were at high risk for an AMI and had indications for cardioprotective medications did not receive them until after AMI. Improved secondary prevention through better prescription of cardioprotective medications in high-risk outpatients with IHD is warranted.

IN MAJORITY OF PATIENTS WHO UNDERGO CARDIOPULMONARY RESUSCITATION (CPR) DURING HOSPITALIZATION OPTION OF CPR IS NOT ADDRESSED AT THE TIME OF ADMISSION. A. Mirza ${ }^{1}$, R. Kad ${ }^{1}$, K. Pachipala ${ }^{1}$, N. Ellison ${ }^{1} ;{ }^{1}$ Geisinger Medical Center, Danville, PA (Tracking ID \#73973)

BACKGROUND: CPR is routinely performed on hospitalized patient in the setting of cardiopulmonary arrest. Majority of patients want to discuss CPR with their physicians and most elderly patients do not desire CPR during acute illness. When told that probability of survival is low after CPR only $5 \%$ patients desired it (Murphy 1994). If the issue of CPR is not 
discussed with patient at the time of admission consent to administer CPR is presumed leading to its administration. Resuscitation in some circumstances may represent a positive violation of an individual's right to die with dignity

Discussion about CPR preference with patients or surrogate decision-maker at the time of admission to hospital will avoid CPR in patients who do not desire it.

METHODS: To test the hypothesis that there is poor communication between admitting physician and patient or surrogate decision-maker regarding CPR at the time of admission we retrospectively studied 100 patients who received CPR in our tertiary care medical center between January 2001 and April 2002. Data was gathered from review of the patient's charts and cardiopulmonary resuscitation records.

RESULTS: Patient's mean age was 66 years (ranged 25 to 86), men comprised 52\% (52/100) of the sample. Although at the time of admission, $72 \%(72 / 100)$ patients were capable of CPR discussion, it was only addressed for $22 \%(22 / 100)$. Of these $41 \%(9 / 22)$ documented the patient's desire for CPR. Family and physician's perspective regarding the CPR decision were also provided in $67 \%(6 / 9)$ and $44 \%(4 / 9)$ charts respectively. The other $59 \%(13 / 22)$ with CPR orders on admission contained no documentation of patient, family or physician's view of CPR. Among 53\% (53/100) patients who were judged to be seriously or critically ill on admission, only $17 \%(9 / 53)$ had CPR status documented in admission orders.

CONCLUSION: 1 . CPR is not discussed with the majority of patients or surrogate decisionmaker at the time of hospital admission.

2. Most patients who undergo CPR in hospital are capable of discussing option of CPR at the time of admission

3. The rationale for CPR orders is very infrequently recorded in the medical records.

PROVIDER ATTITUDES AND MEDICAL ERROR: THE AMBULATORY SAFETY ATTITUDES QUESTIONNAIRE. I. Modak ${ }^{1}$, E.J. Thomas ${ }^{1}$, J.B. Sexton ${ }^{2}$, T.R. Lux ${ }^{1}$, L. Cheng ${ }^{1}$, R.L. Helmreich"; ' ${ }^{2}$ University of Texas Health Science Center at Houston, Houston, TX; ${ }^{2}$ University of Texas at Austin, Austin, TX (Tracking ID \#76313)

BACKGROUND: Errors are common during the delivery of healthcare and experience in aviation may help improve patient safety. In aviation, crewmember attitudes are related to the occurrence of errors and improving these attitudes may decrease error. The University of Texas Safety Attitudes Questionnaire (SAQ) is a psychometrically validated survey for intensive care unit (ICU) providers derived from the aviation survey. Our goal was to develop and administer an ambulatory version of the SAQ.

METHODS: We surveyed 409 providers in a multi-specialty, academic practice in the United States. Items on the ICU SAQ were modified for the ambulatory setting to create a 62 -item survey. We used confirmatory factor analyses to identify scales. Responses were on a five point Likert scale that was converted to 100 -point scale. We report the \% of subjects whose responses were $>75$ (“\% positive"). ANOVA was used to detect differences in scale scores between providers.

RESULTS: Overall response rate was $67.2 \%$ (63\% for physicians and $69 \%$ for ambulatory staff (nurses, administrators, and support staff)). Similar to the ICU version the following six scales were identified: teamwork climate, safety climate, perceptions of management, job satisfaction, working conditions, and stress recognition (Cronbach's alpha ranged from .63-.85 for these factors). For teamwork climate, physician, nurse, management, and support staff $\%$ positive scores were $42 \%, 58 \%, 50 \%$, and $22 \%$ respectively ( $\mathrm{p}=0.01$ ). For safety climate: $46 \%$, $53 \%, 78 \%$, and $47 \%(\mathrm{p}=0.03$ ). For perceptions of management: $43 \%, 63 \%, 72 \%$, and $41 \%$ $(\mathrm{p}=0.23)$. For job satisfaction: $44 \%, 63 \%, 78 \%$, and $50 \%(\mathrm{p}=0.05)$. For working conditions: $28 \%, 32 \%, 56 \%$, and $38 \%(\mathrm{p}=0.31)$. For stress recognition: $10 \%, 11 \%, 6 \%, 0 \%(\mathrm{p}=0.00)$. These differences in scale scores are due to differences in individual items. For example, in safety climate, $89 \%$ of management compared to $64 \%$ of physicians felt encouraged to report any patient safety concerns $(\mathrm{p}=0.03)$. In teamwork climate $75 \%$ of support staff compared to $26 \%$ of nurses reported frequent inability to express disagreement with physicians $(\mathrm{p}<0.001)$. CONCLUSION: Attitudes relevant to medical error differed among ambulatory providers and some provider types had attitudes that may compromise patient safety. It is concerning that providers did not recognize the effect of stress on error. It is also concerning that only $22 \%$ of support staff had a positive attitude about teamwork and that only $46 \%$ of physicians had a positive attitude about safety climate. Measurement of these attitudes in the ambulatory setting may help identify opportunities to improve patient safety.

REVIEW OF APPROPRIATE PERI-OPERATIVE BETA-BLOCKADE USE. $\underline{\text { J. Moriarty }}{ }^{1}$; ${ }^{1}$ VA Connecticut Medical Center, West Haven, CT (Tracking ID \#75526)

BACKGROUND: Peri-operative beta-blockade has been shown to reduce cardiac morbidity and mortality in high-risk patients undergoing major non-cardiac surgery. Little is known about the current rates and appropriateness of use of peri-operative beta-blockade. In order to determine the need for practice improvement at our institition, we reviewed the use of perioperative beta-blockade over a six-month period.

METHODS: We conducted a retropsective chart review of all major non-cardiac operative cases at the Veterans Adminstration Connecticut Medical Center from June to Decemeber of 2001. Patient charts were reviewed for the presence of a medical pre-operative evaluation, eligibility for peri-operative beta-blockade, recommendations regarding the use of betablockade, administration of beta-blockade, and 30 day post-operative cardiac morbidity and mortality. Patients were determined to be eligible for beta-blockade if they had no contraindications to beta-blockade and had coronary disease or at least 2 risk factors for coronary disease

RESULTS: 247 patients underwent major non-cardiac surgery during the study period. A medical pre-operative evaluation was documented in 162 patients $(65.6 \%)$. Beta-blockade was indicated in 180 of 247 patients $(72.9 \%)$, suggested in 84 of the 162 patients $(51.9 \%)$ with a medical pre-operative evaluation, and given in 88 patients (35.6\%). Rates of appropriate betablocker use varied depending on whom performed the pre-operative evaluation. Beta-blockade was suggested in $27 \%, 68 \%$, and $81 \%$ in eligible patients when evaluated by a primary care provider, medical consultation service and cardiology service respectively. Cardiovascular complications occurred in $11.4 \%$ of patients receiving beta-blockade and in $5.7 \%$ of patients who did not receive beta-blockade. 3 deaths $(3.4 \%)$ occurred in the beta-blocker goup compared to 5 deaths $(3.1 \%)$ in those who did not receive beta-blockade. Patients who received beta-blockade were older, mean age 70.5 vs 61.8 years, and were more likley to have coronary artery disease, $67 \%$ vs. $17.6 \%$, when compared to those who did not receive beta-blockade. CONCLUSION: Peri-operative beta-blockade is underused in our high-risk patient population. Strategies directed at improving the use of beta-blockade by primary providers are likely to have a large impact on appropriate use of peri-operative beta-blockade.

A CLINICAL PREDICTION RULE FOR ANGIOTENSIN-CONVERTING ENZYME INHIBITOR-INDUCED COUGH. T. Morimoto ${ }^{1}$, T.K. Gandhi ${ }^{1}$, J.M. Fiskio ${ }^{1}$, A.C. Seger ${ }^{1}$, J.W. So ${ }^{1}$, E.F. Cook ${ }^{1}$, T. Fukui ${ }^{2}$, D.W. Bates ${ }^{1 ;}$ ' ${ }^{1}$ Division of General Medicine and Primary Care, Brigham and Women's Hospital, Boston, MA; ${ }^{2}$ Department of General Medicine, Kyoto University Hospital, Kyoto, (Tracking ID \#74008)

BACKGROUND: Angiotensin-converting enzyme (ACE) inhibitors are effective for many cardiovascular diseases and are widely prescribed, but cough sometimes necessitates their withdrawal.

METHODS: To develop and validate a model that predicts, by using information available at first prescription, whether a patient will develop cough within 6 months, we conducted a retrospective cohort study with derivation and validation sets at outpatient clinics affiliated with an urban tertiary care hospital. The derivation set included 1125 patients and the validation set 567 patients. ACE inhibitor-induced cough was assessed by pre-determined criteria.

RESULTS: In the total cohort, 12\% (202/1692) of patients developed ACE inhibitor-induced cough. Independent multivariate predictors of cough were older age, female gender, nonAfrican American (with East Asian having highest risk), no history of previous ACE inhibitor use, and history of cough due to another ACE inhibitor. Patients with a history of ACE inhibitor-induced cough were 29 times more likely to develop a cough than those without this history. These factors were used to develop a model stratifying patients into four risk groups. In the derivation set, low-risk, average-risk, intermediate-risk, and high-risk groups had a $6 \%$ (24/416), 9\% (43/473), 22\% (45/203), and 55\% (18/33) probability of cough, respectively. In the validation set, $4 \%(7 / 179), 14 \%(37 / 270), 20 \%(22 / 108)$, and $60 \%(6 / 10)$ of patients in these four groups developed cough, respectively.

CONCLUSION: This model may help clinicians predict the likelihood of a particular patient developing cough from an ACE inhibitor at the time of prescribing, and may also assist with subsequent clinical decisions.

THE VALUE OF FAMILY HISTORY INFORMATION COLLECTED FOR CANCER RISK ASSESSMENT IN PRIMARY CARE. H.J. Murff ${ }^{1}$, J.A. Canter ${ }^{1}$, S.K. Syngal ${ }^{2} ;{ }^{1}$ Vanderbilt University, Nashville, TN; ${ }^{2}$ Dana Farber Cancer Institute, Boston, MA (Tracking ID \#75861)

BACKGROUND: Family history data identifies patients that are at increased risks for colon and breast cancers. Several guidelines recommend screening interventions, such as mammography, at an earlier age based on pedigree assessment. In addition, certain individuals may be at very high risk based on family history and may need screening for multiple cancers and benefit from genetic testing. Despite these guidelines, little data exists regarding the quality and use of family cancer history data by primary care providers.

METHODS: We identified 1024 consecutive new patient visit encounters during a 28-week period at a single, large, academic primary care practice. All visit notes were reviewed for documentation regarding a patient's family history of cancer. Using previously published criteria for determining familial cancer risk based on pedigree information, patients were stratified into high, moderate, or average risk categories for breast and colon cancer. A patient was defined as high risk for breast or colon cancer if the disease was identified in one or more first or second-degree relatives with a premature age of onset recorded. A patient was defined as moderate risk if the disease was identified in either one first degree or two-second degree relatives with either a late age of onset or no age recorded. Documentation of a "negative" family history without any cancer diagnosis specified was conservatively considered to be a negative family history for breast and colon cancer.

RESULTS: Of 1024 new-patients, 90\% (918) had some form of family history documented within their medical record and only $63 \%(577)$ had their family cancer history assessed. Of patients with a family cancer history recorded, $79 \%(79 \%)$ were under 50 years of age and $80 \%$ (460) were women. In these 577 patients, colon cancer risk could be determined in $53 \%$ (308) patients. In patients under the age of $50,68 \%$ of men and $55 \%$ of women ( $\mathrm{p}$-value $=0.02$ ) could have colon cancer risk determined based on the information collected. Four individuals were classified as high risk for colon cancer based on their pedigree. Of 30 patients classified as "moderate risk" for colon cancer, 26 (87\%) lacked the necessary information regarding age at diagnosis to determine if they were "high risk". Breast cancer risk could be determined in 54\% (278) of all women. Nineteen women were classified as high risk for breast cancer based on their pedigree. Of the 36 women classified as "moderate risk" for breast cancer, 30 (83\%) lacked the necessary information to determine if they were "high risk".

CONCLUSION: Primary care providers often obtain family history information during new patient visits, however the data are frequently incomplete and cannot be used for accurate cancer risk assessment.

PATIENTS' PERCEPTIONS OF CULTURAL FACTORS AFFECTING THE QUALITY OF THEIR MEDICAL ENCOUNTERS. A.M. Nápoles-Springer ${ }^{1}$, J. Santoyo ${ }^{1}$, K. Houston ${ }^{1}$, E.J. Pérez-Stable ${ }^{1}$, A.L. Stewart ${ }^{1}$; ${ }^{1}$ University of California, San Francisco, San Francisco, CA (Tracking ID \#76394)

BACKGROUND: Due to shortages of ethnically and linguistically diverse clinicians and the growing diversity of patients, cultural factors will assume increasing importance in the provision of quality health care. 
METHODS: The objective of this study was to identify how ethnically diverse patients feel culture affects the quality of their medical encounters. Participants were asked: 1) What does the word 'culture' mean to you? 2) Do you think that culture affects your visits with a doctor? 3) What are the things that your doctors do or do not understand about your culture or health beliefs? 61 African Americans, 45 Latinos and 57 Whites with at least one physician visit in the previous year were recruited from community settings for 19 focus groups. Each text unit (TU) (identifiable continuous verbal utterance) of the focus group transcripts was analyzed using a grounded theory/constant comparison method for content analysis. The proportion of TUs was calculated within each of the 3 areas. Participants' mean age was 48 years, $45 \%$ were women, and $47 \%$ had $\leq$ high school education.

RESULTS: The most salient definitions of culture common to all 3 ethnic groups included a system of values ( $25 \%$ of TUs), customs (17\%), self-identified ethnicity (15\%), and country of origin (11\%). Only Whites and African Americans defined culture as shared experiences (14\%). Only African Americans and Latinos defined culture in terms of discrimination (7\%). In response to whether culture affects the medical encounter, slightly over half of the TUs (52\%) indicated that it did not; the other $48 \%$ of TUs indicating that culture did affect the encounter were made only by African Americans and Latinos. Cultural factors identified by participants from all 3 ethnic groups as influencing the quality of the medical encounter were acceptance of complementary and alternative medicine (17\%), health insurance-based discrimination (12\%), social class-based discrimination (9\%), ethnicity of the doctor (8\%), and age-based discrimination (4\%). Other cultural factors that influenced the encounter mentioned only by Latinos and Whites, included language (13\%), modesty about one's body $(5 \%)$, and submissiveness to physicians (5\%). Ethnicity-based discrimination (11\%), spirituality (2\%) and family (2\%) were cultural factors affecting the encounter specific to the non-White groups. Cultural factors related to language-based discrimination (8\%) and immigration status (5\%) were specific to only Latinos. Physicians' assumptions about patients' health beliefs, social or educational background, treatment preferences, and inability to understand explanations were viewed negatively by respondents from all groups.

CONCLUSION: The provision of quality health care to ethnically diverse patients requires that clinicians be culturally flexible to elicit, adapt and respond to cultural factors in the medical encounter

FOLLOW-UP OF ELEVATED BLOOD PRESSURE IN AN URGENT CARE CLINIC. R. Padilla ${ }^{1}$, C. McDaniel ${ }^{1}$, H. Batal ${ }^{1}$, R. Awad ${ }^{1}$, T. Nickel ${ }^{1}$, P. Mehler ${ }^{1} ;{ }^{1}$ Denver Health, Denver, CO (Tracking ID \#76716)

BACKGROUND: Hypertension is the leading reason for office visits in the United States, yet blood pressure (BP) control in the U.S. is poor as only $27 \%$ of persons with hypertension have their b.p. well controlled with medications. Due to a variety of reasons (lack of transportation, extended hours, lack of insurance), patients are using urgent care centers as their primary source of health care with increasing frequency.

METHODS: We conducted a cross-sectional survey of subjects presenting for care in an urgent care center. Candidate subjects were identified by having a mean b.p. of greater than $140 / 90 \mathrm{mmHg}$ after two separate b.p. checks. Demographic information was collected as well as information about whether or not subjects had a previous history of high b.p. as well as a history of previous or current b.p. medication use. All the subjects were given information about follow up with a primary care provider in order to further assess their b.p. We then did a prospective 3 month chart review to evaluate the subjects b.p. care.

RESULTS: A total of 540 subjects were included in the survey, 362 (67.0\%) of whom had some kind of follow up visit. The total incidence of hypertension in clinic subjects during the study period was $33.0 \% .331(61.3 \%)$ of the subjects had a previous history of elevated b.p., and of those subjects only $160(48.3 \%)$ were currently taking b.p. medication . Factors that predicted any follow up care were: a history of elevated b.p. $(\mathrm{p}=0.008)$, previous $(\mathrm{p}<0.0001)$ or current $(\mathrm{p}$ $<0.0001)$ use of b.p. medication, having a regular source of health care $(\mathrm{p}<0.0001)$ and having health insurance $(\mathrm{p}<0.0001)$. Gender, race, primary language and level of education did not have a significant influence on follow-up rates. Table 1 summarizes 2 groups of elevated blood pressure for the entire population and by the presence or absence of follow-up data.

CONCLUSION: Patients who have significantly elevated b.p. in an urgent care center are prime candidates for the initiation of or titration of b.p. medication. One should seize the opportunity to treat these patients because many of these patients will be lost to follow-up or will mistakenly opt to discontinue their medication.

\section{Table 1. Groups Of Elevated B.P. And Clinic Follow-Up}

$\begin{array}{lcccc}\text { B.P. Group } & \begin{array}{c}\text { Entire Population } \\ (\mathbf{n}=\mathbf{5 4 0})\end{array} & \begin{array}{c}\text { Any Follow-Up } \\ (\mathbf{n}=\mathbf{3 6 2})\end{array} & \begin{array}{c}\text { No Follow-Up } \\ (\mathbf{n}=178)\end{array} & \text { P value } \\ 140-159 / 90-99 & 352(65.2 \%) & 224(61.9 \%) & 128(71.9 \%) & 0.02 \\ \geq 160 / \geq 100 & 188(34.8 \%) & 138(38.1 \%) & 50(28.1 \%) & \end{array}$

DOCUMENTATION OF PHYSICIAN RECOMMENDATIONS FOR INFLUENZA AND PNEUMOCOCCAL VACCINATIONS IN ELDERLY PATIENTS. M.A. ParkulO ${ }^{1}$, R.J. Presutti ${ }^{1}$, W.E. Haley ${ }^{1}$, K. Portell ${ }^{1}$, L. Anderson ${ }^{1}$; ${ }^{1}$ Mayo Clinic, Jacksonville, FL (Tracking ID \#76513)

BACKGROUND: Influenza and Pneumococcal pneumonia cause significant mortality and morbidity, especially in the elderly. Immunization guidelines for 2001 recommended annual Influenza vaccination and a one time Pneumococcal vaccination for all patients over the age of 65. Previous surveys of Medicare beneficiaries have shown that up to $20 \%$ of respondents who did not receive appropriate vaccinations were unaware or not informed of the recommendations by their physician.

METHODS: Our institution is totally electronic in the outpatient setting. All clinical notes are dictated and transcribed or entered by direct typing. We have established a data retrieval system whereby we can search for specific content in each clinical document type. We used this system to query every history and physical note entered at our multi-specialty institution in the year 2001 on patients age 65 and older. We queried the notes using the terms Influenza or flu and shot or immunization or vaccin* (which would capture vaccine or vaccination) to search for documentation of Influenza vaccination review or recommendations. Similarly we queried the notes using the terms pneumo* (which would capture pneumovax or pneumococcal) and pneumonia or pneumococcal.

RESULTS: 33,023 history and physical notes entered in 2001 were queried using the above criteria. Documentation meeting our criteria for mentioning Influenza vaccination was found in 4, 476 documents $(13.6 \%)$. Documentation meeting our first criteria for mentioning pneumonia (pneumo*) was found in 10,901 documents ( $33.0 \%$ ). Documentation meeting our second criteria for mentioning pneumonia (pneumonia or pneumococcal) was found in 6,114 $(18.5 \%)$ of documents.

CONCLUSION: At our institution there was very poor documentation which would be consistent with Influenza or Pneumococcal vaccination review or recommendations by physicians. We are initiating appropriate interventions to improve compliance. As computerized systems become more adept at analyzing clinical notes, proper physician documentation will become more important to demonstrate appropriate quality of care. These systems should and will be used to monitor compliance with quality initiatives.

VIGNETTES: AN INNOVATIVE TECHNOLOGY FOR MEASURING QUALITY IN DIVERSE POPULATIONS. J.W. Peabody ${ }^{1}$, J. Luck ${ }^{2}$, P.A. Glassman ${ }^{3}$, S. Jain ${ }^{4}$, J. Hanson ${ }^{5}$, M.X. Spell $;{ }^{1}$ SF VAMC c/o IGH, San Francisco, CA; ${ }^{2}$ UCLA, Los Angeles, CA; ${ }^{3}$ VA, West LA, Los Angeles, CA; ${ }^{4}$ VAMC, San Francisco, CA; ${ }^{5}$ California Pacific Medical Center, San Francisco, CA; ${ }^{6}$ Kaiser Permanente, Los Angeles, CA (Tracking ID \#75805)

BACKGROUND: Measuring the quality of care is both a health services priority and a technical challenge. This challenge is heightened when comparisons are made across diverse populations and divergent health systems. Measurements must be valid, reliable and affordable. Computerized vignettes - an innovative new measurement system — can be used to measure the quality of care across in these settings. This study validates vignettes against standardized patients (SPs) and the usual measure of quality, medical records abstraction.

METHODS: We prospectively compared three measurement methods on a random sample of physicians at 4 different institutions. Computerized vignettes for 8 highly prevalent outpatient conditions were developed. A case begins with a typical problem. Physicians respond by typing in questions and "taking the history." Programmed responses are provided in real time. This process is repeated for the domains of physical examination, testing, diagnosis and treatment. Second, we validated the vignettes by having 41 trained SPs present unannounced with the identical 8 conditions into the different health care systems located in California. The SPs completed a checklist after completing each visit. Third, we abstracted the medical record from each visit. For each of the 3 methods, 25-30 identical scoring criteria were defined and used to score each of the 8 cases. Vignettes were scored by both a trained abstractor and an automated word recognition program. In total, sixty primary care generalists from 2 VA sites and 2 nearby non-VA sites saw a total of 480 cases. We then compared the quality of care scores, recorded as $\%$ correct, for each method using an ANCOVA model.

RESULTS: Scores of quality as measured by vignettes $(64 \%)$, were closer to the gold standard of SPs $(71 \%)$ than the medical records $(57 \%, \mathrm{p}<.005)$. Vignettes accurately captured the variation in the quality of care between sites. Of particular note is that the within-site variation in the quality of care exceeded the between-site variation $(\mathrm{p}<.005)$. This pattern persisted across domains, case, site, level of training and case complexity $(\mathrm{p}<.005)$. Automated scoring of vignettes, validated against the trained abstractor, reduced scoring costs by $80-90 \%$ and eliminated interrater variability.

CONCLUSION: Vignettes appear to be more a valid and reliable method for measuring the quality of physician practice than medical record abstraction. Vignettes appear to reflect actual clinical practice and constitute an innovative, low cost method for evaluating the quality of care. Since they eliminate case mix variation, vignettes are particularly helpful for comparisons in diverse patient populations and divergent health care systems.

RELATION OF DIABETES-SPECIFIC KNOWLEDGE TO SELF-CARE ACTIVITIES, AMBULATORY CARE PROCESSES, AND METABOLIC OUTCOMES IN ADULTS WITH DIABETES. S.D. Persell ${ }^{1}$, N.L. Keating ${ }^{1}$, M. Landrum ${ }^{2}$, B.E. Landon ${ }^{2}$, J.Z. Ayanian ${ }^{1}$, E. Guadagnoli ${ }^{2}$; ${ }^{1}$ Brigham and Women's Hospital, Boston, MA; ${ }^{2}$ Harvard University, Boston, MA (Tracking ID \#74887)

BACKGROUND: Patient education and self-management training are emphasized as important parts of diabetes treatment. Yet, educational interventions and diabetes knowledge have not been consistently associated with improved diabetes outcomes. We sought to examine the relationship of patients' diabetes-specific knowledge with their processes and outcomes of diabetes care.

METHODS: We conducted a mail survey of adults with diabetes in 3 managed care plans in Minnesota in order to assess their diabetes knowledge (using an 8-point scale) and self-care activities (following a diabetes diet, self-monitoring of blood glucose $\geq$ once per week (SMBG), and exercise $\geq 3$ times per week). We assessed 5 processes of care - retinal examination, foot examination, low density lipoprotein cholesterol (LDL-C) testing, hemoglobin A1c (HbAlc) testing, and urine microalbumin testing - and 3 metabolic outcomes - HbAlc $<=9.5 \%$, last blood pressure $<140 / 90$, and LDL-C $<130 \mathrm{mg} / \mathrm{dl}$ - using ambulatory medical records for 1998. We used logistic regression to assess the relationship between diabetes-specific knowledge and these outcomes, adjusting for demographic and clinical variables.

RESULTS: The survey was completed by 670 patients (response rate $65.5 \%$ ). The mean age was 60.5 (SD 14.6) years. $68.3 \%$ reported following a diet, 78.3\% did SMBG, $49.8 \%$ reported exercise, $80.0 \%$ had retina exams, $34.0 \%$ had foot exams, $47.2 \%$ had LDL-C testing, $88.2 \%$ had HbAlc testing, 37.3\% had microalbumin testing, $78.4 \%$ had $\mathrm{HbAlc}<=9.5,54.0 \%$ had blood pressure $<140 / 90$, and $30.0 \%$ had LDL-C $<130$. The mean score on the 8-point knowledge scale was 5.4 (SD 1.9) (Cronbach's alpha $=0.7$ ). In adjusted analyses, a 1-point increase on the knowledge scale was associated with following a diet (OR 1.20, p = 0.002), 
SMBG at least weekly (OR 1.25, $\mathrm{p}=0.001$ ), and exercise at least 3 times weekly (OR 1.14, $\mathrm{p}=0.02$ ), but not with processes of ambulatory care (ORs $0.96-1.11, \mathrm{p}>0.08$ ), or metabolic outcomes (ORs 1.06-1.07, p > 0.2). Following a diet, SMBG, and exercise were not significantly associated with improved metabolic outcomes $(\mathrm{p}>0.15)$.

CONCLUSION: In a large insured population, knowledgeable patients with diabetes were more likely to perform self-care activities but not more likely to receive recommended processes of care or achieve desired metabolic outcomes. Our results suggest patient knowledge is associated with self-care but not other aspects of diabetes care. Further studies should examine if increased emphasis in diabetes patient education on specific processes of ambulatory care leads more patients to obtain recommended care and achieve desired outcomes.

PRESCRIPTIONS FOR HEALTH INFORMATION IMPROVE QUALITY OF CARE. R.G. Pinckney ${ }^{1}$, J. Light ${ }^{1}$, P. Winstead-Fry ${ }^{1}$; ${ }^{1}$ University of Vermont, Burlington, VT (Tracking ID \#75711)

BACKGROUND: Prescriptions for health information are a novel intervention that have not been evaluated.

METHODS: This is a prospective controlled trial of a prescription for a health information packet written by primary care providers. Patients with uncontrolled or newly diagnosed diabetes, hypertension, or hypercholesterolemia were enrolled from nine primary care practices in northwestern Vermont. We measured the proportion of patients whose $\mathrm{HgAlc}$, blood pressure, or cholesterol had reached targets suggested by guidelines.

RESULTS: 153 patients met eligibility criteria. $52 \%$ of patients who filled a prescription reached the goal of their therapy while only $28 \%$ of the control group reached the goal, $\mathrm{P}=.03$. After adjusting for gender and prior disease control, the odds ratio and $95 \%$ confidence interval for reaching goal of therapy after filling the prescription was 2.85 (1.10-7.39).

CONCLUSION: Prescriptions for information improve the quality of care for patients with diabetes, hypertension, and hypercholesterolemia.

ANTIDEPRESSANT PRESCRIBING FREQUENCY IS ASSOCIATED WITH TREATMENT ADEQUACY RATES AMONG PRIMARY CARE PHYSICIANS. P. Pirraglia ${ }^{1}$, J.B. Weilburg ${ }^{1}$, K.M. O'Leary ${ }^{1}$, D.E. Singer ${ }^{1}$, J.B. Meigs ${ }^{1}$; ${ }^{1}$ Massachusetts General Hospital, Boston, MA (Tracking ID \#76128)

BACKGROUND: Physicians who frequently perform certain surgical procedures tend to have better outcomes than do those less frequently performing the same procedures. We hypothesized that a similar 'volume-outcome' relationship might be found among primary care physicians (PCPs) prescribing antidepressant (AD) medications, with PCPs more frequently prescribing $\mathrm{ADs}$ having higher rates of adequate treatment (treatment concordant with minimum guideline standards for dose and duration).

METHODS: We reviewed AD prescriptions written over a 1.75 year period by PCPs affiliated with Massachusetts General Hospital (MGH) for patients with selected commercial insurance. Physicians who treated $<2$ patients during this period were excluded from analysis. Frequency of prescribing was defined as the number of patients prescribed ADs, and stratified into tertiles: low ( 2 to 11 patients), medium (12 to 25$)$, and high (>25-102). Adequate treatment was defined by refill histories indicating use of at least a minimum likely-effective average daily dose continuously for at least 90 days. Prescriptions for low doses of tricyclic ADs or trazodone were excluded from analysis. The treatment adequacy rate was the number of patients per PCP with an adequate course of treatment divided by the number of the PCP's patients who filled AD prescriptions. Linear regression was used to compare adequacy rates among the frequency strata. RESULTS: Data was available for $163 \mathrm{MGH}$ affiliated physicians. The median volume of treated patients over 1.75 years was 17 (range $2-102$ ). The overall mean adequacy rate was $45.2 \%$ (sd $15 \%$, range 0 to $81 \%$ ). The adequacy rate for the low frequency group was $41.4 \%$ (sd $18.6 \%$ ), $45.7 \%$ ( $\mathrm{sd} 14.8 \%$ ) for the medium group, and $48.6 \%$ (sd $9.0 \%$ ) for the high frequency group, indicating a significant positive relationship between frequency of $\mathrm{AD}$ treatment and adequacy ( $\mathrm{p}<0.04$ for linear trend). The low group had a significantly lower adequacy rate compared to the high group $(\mathrm{p}<0.02)$.

CONCLUSION: PCPs prescribing ADs more frequently in this population during this period had higher adequacy rates than those prescribing $\mathrm{AD}$ less frequently. Quality improvement efforts to improve $\mathrm{AD}$ prescribing practices might target low-volume prescribers for special emphasis to increase familiarity with guidelines for use of these medications.

PHYSICIANS' PERCEPTIONS TOWARD ELECTRONIC COMMUNICATION WITH PATIENTS. L. Pizziferri ${ }^{1}$, A. Kittler ${ }^{1}$, L.A. Volk ${ }^{1}$, J. Hobbs ${ }^{1}$, Y. Jagannath ${ }^{1}$, J.S. Wald ${ }^{1}$, B. Middleton ${ }^{2}$, D.W. Bates; ${ }^{1}$ Partners HealthCare System, Inc., Wellesley, MA; ${ }^{2}$ Brigham and Women's Hospital, Boston, MA (Tracking ID \#76446)

BACKGROUND: Despite the widespread use of email, electronic communication between physicians and patients is not part of the standard physician-patient relationship. Increased use of email may improve physician-patient communication, which is associated with improved patient satisfaction and health status. Evaluating email communication in this context is vital to minimizing potential risks and maximizing benefits to physicians and patients. We evaluated email use between physicians and patients, and physicians' perceptions of the value and issues surrounding this form of communication in order to identify facilitators.

METHODS: A paper-based survey was sent to 94 primary care physicians in an integrated delivery system. We assessed the role email plays in a typical workday and the barriers to increasing email use with patients. A structured interview was also performed with a subset of respondents that reported the highest percentage of email use with patients. The interviews evaluated how physician-patient email contact is typically initiated, approaches to managing emails such as triaging and guidelines, the content of emails, and physician views on how email communication could be improved.

RESULTS: Seventy-one of 94 physicians responded to the survey for a response rate of $76 \%$. Seventy percent of physicians use email with their patients, but the majority do so with only 1$5 \%$ of their patient panel. The main reported barriers to physician-patient email related to workload, security and payment. Interviews with 10 of the survey respondents revealed that the actual time spent and quantity of emails is minimal; physician contact with patients ranges from $2-5$ patients on a typical day, with an average of two emails per patient (received and sent). Patients generally initiated email contact. Guidelines regarding the use of email are seldom given to patients. Most emails from patients relate to prescription refills, referrals, appointments, test results and questions relating to personal healthcare. In all interviewed cases physicians are the first to read email and responded directly.

CONCLUSION: Electronic communication between physicians and patients has great potential to improve communication, but needs to go beyond the basic functionality of typical email. Our findings indicate that with adequate pre-screening, triage, and reimbursement mechanisms physicians would be open to substantially increasing electronic communication with patients. Formal guidelines for patients and physicians and a measured approach to introducing electronic communication to patients would capitalize on potential gains in efficiency and quality of care.

'I WISH I HAD SEEN THIS RESULT EARLIER': PROBLEMS IN THE TEST RESULT MANAGEMENT SYSTEMS IN PRIMARY CARE PHYSICIANS' OFFICES. E.G. Poon ${ }^{1}$ T.K. Gandhi ${ }^{1}$, T. Sequist ${ }^{1}$, D.R. Webber ${ }^{1}$, H.J. Murff ${ }^{1}$, A.S. Karson ${ }^{2}$, D.W. Bates ${ }^{1} ;{ }^{1}$ Brigham and Women's Hospital, Boston, MA; ${ }^{2}$ Massachusetts General Hospital, Boston, MA (Tracking ID \#74380)

BACKGROUND: Failure to review and follow-up on outpatient test results in a timely manner is a patient safety and malpractice concern. Therefore, we sought to assess the frequency of delays in test result review and identify aspects of current test result review systems that are associated with delays.

METHODS: We surveyed 176 attending level primary care physicians working in 22 clinics affiliated with 2 large urban teaching hospitals. To assess delays in reviewing test results, we asked physicians to report the number of times over the previous 2 months that they had reviewed results they 'wished they had reviewed earlier'. To describe the current test result review systems, we asked physicians whether they had a system to verify the completion of tests ordered, and whether a staff member was dedicated to screen incoming test results for serious abnormalities. We also asked the amount of time physicians spent per clinic day on test result management. We built clustered, multiple logistic regression models using SAS GENMOD to identify aspects of the current result management systems that were associated with delays in reviewing test results.

RESULTS: 107 physicians responded to our survey (response rate $=61 \%$ ). Overall, $79 \%$ of physicians reported reviewing at least one result over the previous 2 months that they 'wish they had reviewed earlier.' On average, physicians reported that delays occurred 2.5 times over a 2 -month period. $33 \%$ of physicians had a system to verify the completion of tests ordered, and $50 \%$ of physicians had a staff member dedicated to screen test results for serious abnormalities. The average physician spent 72 minutes per clinic day on managing test results. In a clustered multiple logistic regression model, physicians who practiced 4 or more half-day sessions per week $(\mathrm{OR}=4.31, \mathrm{p}=0.02)$, and those who had a staff member dedicated to screen test results for serious abnormalities $(\mathrm{OR}=4.12, \mathrm{p}=0.004)$ were more likely to report delays with reviewing test results at least once over the previous 2 months.

CONCLUSION: Failure to review outpatient test results in a timely manner is a common problem, despite the substantial amount of time physicians spend on managing results. Physicians who carry a heavier weekly patient load and those who rely on staff members to screen results for abnormalities may be at higher risk of missing important test results. These results highlight the need for more reliable and efficient test result management systems to address this patient safety problem.

MASTER SCHEDULING TEMPLATE: 5 STEPS TOWARD IMPROVING ACCESS IN THE BREAST DIAGNOSTIC CLINIC. S. Pruthi ${ }^{1}$, L. Litwiller ${ }^{1}$, J. Broers ${ }^{1} ;{ }^{1}$ Mayo Clinic, Rochester, MN (Tracking ID \#74603)

BACKGROUND: Question: How could the Breast Diagnostic Clinic provide timely, efficient, equitable access to the breast practice?

Objectives:

1) To understand the true demand for their service

2) Improve consistency in physician- staffing levels

3) Better utilization of the nurse practitioner (NP)

4) Improve financial performance

Description of program/intervention:

The Breast Diagnostic Clinic ( BDC) and Breast Imaging (BI) moved into Mayo Clinic's new Gonda Building, a major "practice integration project" on the Mayo Campus. Their intent was to develop an integrated breast practice.

In preparation for this integration effort, in 2001, BDC and BI joined a national collaborative, organized by the Institute For Healthcare Improvement (IHI), the Idealized Design of Clinical Office Practice (IDCOP).

At the heart of IDCOP is the focus on several themes for innovation:

Patient access to care and information

Interaction between the patient and the care team

Reliable provision of effective care

Vitality, as indicated by a practice that is "financially viable, innovative, and a great place to work"

METHODS: What steps were needed to improve access in the Breast Diagnostic Clinic (BDC)?

Step 1- Understand the demographics and needs of the BDC patients?

Where do the patients come from

Age distribution

Payer source

Step 2- Understand demand for BDC services

How many patients want to be seen in day? 
What is the variation from day to day, by season?

Understand the role of the multidisciplinary model and consultations with Surgery, Radiation Oncology, Medical Oncology.

In corporate the results of the patient satisfaction survey

Step 3- Understand healthcare team roles

Healthcare team comprised of MD, NP, Clinical Nurse Specialists, clinical assistants, secretarial staff

Step 4- Understand capacity of the practice

What workload can reasonably be accomplised in a day?

How many hours per day/week are providers in the clinic AND available to see patients?

Step 5-Develop a master schedule that optimizes the match between demand and capacity Master Scheduling. What is it?

A high-level view of health care team's schedule (not patient)

One tool to begin to match demand with capacity

Mechanism to assure optimal use of practice resources

RESULTS: Findings to date

Step 1

Majority of patients come from within the 500 mile radius

Medium age is $40-61$ years

Payor source $-61 \%$ are commercial and contract

Step 2

Demand not only included the new consultations, and established patients but "extras, walk-ins and checkers"

Patients with a urgent diagnosis preferred appointment with 1-3 days

Step 3

Develop a model with the NP where new patients can be seen by the NP and supervised by the physician- increase access and number of new patients seen each day

Step 4

Inconsistent capacity in BDC compromised patient access to breast practice

Unpredictable schedules with multiple, part-time physicians: competition for their time (education, research, administrative activities)

Unveven capacity in BDC affected downstream access

Increase 48 hour availability

Some appointment slots inappropriately used ( return PM slots appear to go unused)

Step 5

Master Schedule provided a full year schedule available electronically on a central server

Advanced notice allows desk to staff to workload

Patients may be scheduled up to 2 months in advance

Increased flexibility to allow for absences

Can be modified up to 8 weeks in advance

Management of 3 calendar templates: Physician, NP/MD collaborative model, rotation between BDC and Breast Cancer Clinic

Improves consistency of downstream access

CONCLUSION: Key Lessons Learned

Increased capacity through use of MD/NP collaborative model

Master Schedule is a significant change from traditional practice ( paper to electronic) and can be actively managed and monitored for changes

Thorough understanding of breast practice has allowed BDC to plan and predict demand and resource utilization

Analysis of the breast practice prepared BDC to build a master schedule

Knowledge about patient expectation and the "downstream" effects of the practice can be anticipated and planned for

Modalities to be used to demonstrate Innovation at meeting

Excel Program and the Electronic Master Schedule and other potential uses in the future ie- to monitor productivity

VALIDITY OF CODING PROCEDURES IN ICD-9-CM ADMINISTRATIVE DATA. H. Quan ${ }^{1}$, G.A. Parsons ${ }^{2}$, W.A. Ghali ${ }^{1} ;{ }^{1}$ University of Calgary, Calgary, Alberta; ${ }^{2}$ Calgary Health Region, Calgary, Alberta (Tracking ID \#76041)

BACKGROUND: Administrative hospital discharge data are widely used to assess quality of care and health services utilization in patients undergoing certain procedures. Given that little is known about the validity of procedure coding, we conducted a detailed chart review to evaluate the accuracy and completeness of information on procedures in administrative data. METHODS: We randomly selected 1200 hospital separations between April 1, 1996 and March 31, 1997 from administrative discharge data of adult acute care hospitals in Calgary, Alberta, Canada. Each separation coded in administrative data contains up to 10 procedure codes. The corresponding medical charts were reviewed for determining presence or absence of procedures. We assessed agreement between administrative data and chart review data (criterion standard) for a selection of major general surgical procedures that are usually performed in operating rooms and minor procedures that are routinely performed on medical and surgical wards or in radiology departments.

RESULTS: The agreement between the two databases varied greatly across 35 procedures studied. The sensitivity (quantifying the accuracy of coding presence of procedures in administrative data when these are present in the chart data) ranged from 0 to $94 \%$. Of the six major procedures, validity of coding was generally good, with 5 procedures having coding sensitivity of $69 \%$ and over and only one (lysis of peritoneal adhesion) with a low sensitivity of $41 \%$. In contrast, many minor procedures had low sensitivities. Of the 29 minor procedures studied, sensitivity was lower than $50 \%$ for 15 procedures, between 50 to $79 \%$ for 10 , and $80 \%$ and over for 4.

CONCLUSION: Administrative data appear to be quite valid for detection of major surgical procedures. However, the data lack validity for many minor procedures. If administrative data are ever to be used to study utilization of minor procedures in hospitalized patients, special coding policies will need to first be developed to improve capture of minor procedures for which ICD-9-CM codes exist, but for which coding is incomplete.
COMPARING PERCEPTIONS OF ACCESS IN VA AND NON-VA PRIMARY CARE. K.L. Ravenell ${ }^{1}$, V.J. McDonald ${ }^{1}$, D.A. Asch ${ }^{1}$, C.A. Henry ${ }^{1}$, K.M. Fosnocht ${ }^{2}$, J.J. Murphy ${ }^{1}$ J.A. Shea ${ }^{1} ;{ }^{1}$ CHERP- PVAMC, Philadelphia, PA; ${ }^{2}$ University of Pennsylvania, Philadelphia, PA (Tracking ID \#75120)

BACKGROUND: Access is an important feature of health services, and a significant reason why people do not get quality health care. In many settings, African Americans have reduced access compared to Caucasians; however, racial differences in access are reported to be mitigated within the VA system where barriers to care are minimized. We hypothesized that fewer racial differences would exist within the VA.

METHODS: Patients were recruited from primary care clinic waiting areas of the University of Pennsylvania Health System (UPHS) and the Philadelphia Veterans Affairs Medical Center (PVAMC). Patients provided demographic information and completed the 1999 National Performance Data Feedback Center (NPDFC) Ambulatory Care Satisfaction Survey. The 62 items on the NPDFC are grouped into nine specific and two overall subscales. The access subscale has 7 items. For all items, answers that indicated a problem were scored " 1 " and those that did not indicate a problem were scored "0," thus higher scores indicate more dissatisfaction.

RESULTS: These results are based on responses for 603 men, $86 \%$ from the PVAMC. $56 \%$ of the patients were African-American, 59\% were between 45 and 64 years old and $39 \%$ completed high school. Mean overall access scores were worse in the PVAMC than UPHS $(.31$ vs $.26, \mathrm{p}=.0001)$. Similarly, patients in the PVAMC had significantly worse mean scores than patients in UPHS for three items: calling for an appointment (.50 vs .27, p<.0001), waiting in line to check-in $(.29 \mathrm{vs} .10, \mathrm{p}=.0006)$, and waiting too long in the waiting room $(.60 \mathrm{vs} .37, \mathrm{p}<$ .0001). There were no differences in mean overall access scores between African-Americans and Caucasians at the PVAMC $(\mathrm{p}=.66)$ or UPHS $(\mathrm{p}=.65)$, but differences existed on an item level within the PVAMC. Mean access scores were worse for African-Americans than Caucasians for calling for an appointment $(.541$ vs $.446, \mathrm{p}=.0429)$ but were better for waiting after scheduled appointment time to be seen $(.188 \mathrm{vs} .306, \mathrm{p}=.0034)$. In a stepwise regression, visit site was the only significant predictor of satisfaction with access when adjusting for age, race, and $\operatorname{sex}(\mathrm{p}=.0003)$

CONCLUSION: Access reflects not only eligibility and enrollment, but also ability to use health care systems effectively once enrolled. For patients already enrolled within the VA and wthin UPHS, African Americans report similar access as Caucasians. However, satisfaction with access is lower in a VA than in the UPHS system. While financial barriers are minimized at the VA, there are clearly other barriers to access that feature more prominently than at a non-VA system.

IMPROVING QUALITY OF PRIMARY CARE FOR PANIC AND GENERALIZED ANXIETY DISORDER (PD/GAD). B.L. Rollman ${ }^{1}$, B.H. Belnap ${ }^{1}$, W. Gardner ${ }^{1}$, C.F. Reynolds ${ }^{1}$, H. Schulberg ${ }^{1}$, K. Shear ${ }^{1}$; ${ }^{1}$ University of Pittsburgh, Pittsburgh, PA (Tracking ID \#76151)

BACKGROUND: PD and GAD are prevalent in primary care practice and often inadequately recognized and treated by PCPs. We performed a clinical trial to test the effectiveness of a telephone-based collaborative care strategy for improving the quality of primary care for PD/ GAD.

METHODS: We used the PRIME-MD to identify patients with PD and/or GAD at four clinics sharing a common electronic medical record (EMR) system. Protocol-eligible patients reported a baseline Hamilton Rating Scale for Anxiety (HRS-A) $>13$ or Panic Disorder Severity Scale (PDSS) $>6$. We informed patients' PCPs of these findings via EMR and asked them to electronically indicate agreement/disagreement with the diagnosis. Following PCP agreement, we randomized patients to either telephone-based care management for PD/GAD, or to a "usual care" control condition. The care manager assessed patient's treatment preferences (pharmacotherapy, counseling, or specialty referral), imparted self-management skills using a workbook, and provided periodic feedback and treatment recommendations to the PCP via EMR. Telephone assessments were conducted at 2-, 4-, 8-, and 12 months following recruitment.

RESULTS: Between 7/00 and 4/02, we recruited 181 patients who completed the PRIME-MD and met all study eligibility criteria ( $43 \% \mathrm{GAD}, 11 \% \mathrm{PD}, 46 \% \mathrm{PD} / \mathrm{GAD})$. Their mean age was 43 (range 19-63), 82\% were female, 97\% Caucasian, and 67\% had co-morbid depression. PCPs agreed with $99 \%$ of the anxiety diagnoses. At baseline, mean scores for PDSS, HRS-A, and SF-12 MCS were 8.2, 19.9, and 30.4, respectively. HRS-A and PDSS differed by type of anxiety disorder $(\mathrm{p}<0.001)$ but not SF-12 MCS $(\mathrm{p}=0.06)$. To date, $96 \%$ of patients completed one or more follow-up assessments. Random-effects regressions demonstrated a better rate of improvement in anxiety severity (HRS-A $b=-0.29$ points/month, $\mathrm{p}=0.02 ;$ PDSS $\mathrm{b}=-0.17$ points/month, $\mathrm{p}<0.03$ ) and mental health-related quality of life (SF-12 MCS $b=+0.51$ points/ month; $\mathrm{p}<0.02$ ) for patients randomized to care management vs. usual care after controlling for gender, race, age, type of anxiety disorder, and co-morbid depression.

CONCLUSION: A telephone-based collaborative care model for treating PD and GAD can improve clinical outcomes.

REFINEMENT AND VALIDATION OF THE AHRQ PATIENT SAFETY INDICATORS (PSI). P.S. Romano ${ }^{1}$, J. Geppert ${ }^{2}$, S. Davies ${ }^{3}$, K. McDonald $^{3}$, M. Miller ${ }^{4}$, A. Elixhauser ${ }^{5}$; ${ }^{1}$ University of California, Davis, Sacramento, CA; ${ }^{2}$ National Bureau for Economic Research, Stanford, CA; ${ }^{3}$ Stanford University, Stanford, CA; ${ }^{4}$ Johns Hopkins Childrens Center, Baltimore, MD; ${ }^{5}$ Agency for Healthcare Research and Quality (AHRQ), Rockville, MD (Tracking ID \#76539)

BACKGROUND: Recent reports have focused national attention on preventable medical errors. However, researchers and policy-makers currently have few tools with which to study the epidemiology of patient safety. The UC-Stanford Evidence-based Practice Center worked with AHRQ to refine and validate a pilot set of Patient Safety Indicators (PSIs) based on routinely collected hospital administrative data.

METHODS: The project consisted of five phases. First, we systematically reviewed the research literature to identify candidate indicators of iatrogenic complications and to collect information about their coding and construct validity. Second, we evaluated the face validity of 
candidate indicators by convening 11 panels of 5-9 expert clinicians nominated by 28 professional organizations, and applying an adaptation of the RAND/UCLA Appropriateness Method. Third, we consulted coding experts to confirm the ICD-9-CM definition of each indicator. Fourth, we empirically evaluated promising indicators using the Healthcare Cost and Utilization Project (HCUP) 1995-97 State Inpatient Databases, adjusting for age, gender, comorbidities, DRG clusters, and selected interactions. Multivariate signal extraction and factor analytic methods were used to understand hospital-level variation. Fifth, we wrote and tested SAS code that researchers can use to estimate PSI rates based on their own data. RESULTS: We identified 34 candidate indicators, after excluding those previously shown to have questionable coding or construct validity. Based on second-round panel ratings, 22 indicators had acceptable face validity. We dropped 2 indicators due to operational concerns and empirically evaluated the remaining 20 (plus 17 lower-rated "experimental" indicators). Of these 20 indicators, 2 (transfusion reaction, foreign body left in) had minimal hospital-level variation. All but 5 of the 18 remaining indicators had signal-to-noise ratios exceeding 0.5 . Year-to-year hospital-level correlations exceeded 0.4 for 11 indicators and 0.6 for 4 indicators (including decubitus ulcer and infection due to medical care). Factor analysis suggested 2 underlying constructs: obstetric, catheter-related, and technical complications versus postoperative complications.

CONCLUSION: To evaluate patient safety interventions and identify best practices, researchers need reliable and valid outcome measures. Through literature review, expert panel ratings, and empircal analyses, we identified and refined 20 indicators that hospitals, state data organizations and others can use to screen for potential patient safety problems and enhance surveillance and quality improvement activities.

EFFECTIVENESS OF EVIDENCE-BASED QUALITY IMPROVEMENT FOR DEPRESSION. L.V. Rubenstein ${ }^{1}$, L.S. Meredith ${ }^{2}$, L.E. Parker ${ }^{2}$, N.P. Gordon ${ }^{3}$, M.L. Lee ${ }^{4}$, S. Hickey ${ }^{2} ;{ }^{1}$ VA Greater Los Angeles, Los Angeles, CA; ${ }^{2}$ RAND, Santa Monica, CA; ${ }^{3}$ Kaiser Permanente Division of Research, Oakland, CA; ${ }^{4}$ UCLA, Los Angeles, CA (Tracking ID \#75505)

BACKGROUND: Healthcare organizations depend upon quality improvement methods to develop or adopt care improvements for complex illnesses like depression. Classical continuous quality improvement (CQI), however, has not been effective for this condition in previous studies. This study aimed to evaluate the effectiveness of a modified version of CQI called Evidence-Based Quality Improvement (EBQI) in improving the structure, process and outcomes of care for major depression in primary care.

METHODS: We randomized 6 Kaiser Permanente $(\mathrm{KP})$ and 3 VA primary care practices with a total of 176 clinicians in matched triplets to EBQI Central Team (CT) and EBQI Local Team (LT) or to usual care. EBQI emphasized providing QI teams with literature and tools to support the design and implementation of evidence-based depression improvement programs (DIPs). The Central Team (CT) approach emphasized design by regional experts and the local team (LT) approach emphasized design by local clinicians. Data sources included qualitative evaluation of team DIPs with national expert ratings of evidence-based design and implementation (EB), a self-administered pre-intervention survey of consecutive patients, and a telephone administered post-intervention survey of consecutive patients with major depression with follow-up at six and twelve months. Our outcome measures included depression remission and appropriate depression care (yes/no variables), as well as health-related quality of life and satisfication with communication and treatment choice (continuous variables).

RESULTS: The pre-intervention sample showed no significant differences between experimental and control practices in patient characteristics or in rates of depression diagnosis. Four of 6 EBQI practices implemented EB DIPs; 2 LTs and no CTs implemented non-EB DIPs. The post-intervention sample showed trends toward reductions in depression 12 months after baseline in intervention clinics $(\mathrm{p}=.18$, OR $.60 \pm 1.4)$, and in CTs $(\mathrm{p}=.12$, OR $.51 \pm 1.5)$ but less so in LTs $(\mathrm{p}=.34$, OR $.70 \pm 1.5)$. EB DIPs but not non-EB DIPs showed significant reductions in depression ( $\mathrm{p}=.03$, OR .45 \&plusmn 1.3); improvements in social activities $(\mathrm{p}=.03)$, patient satisfaction with communication and treatment choice $(\mathrm{p}=.04)$; and greater likelihood of appropriate depression treatment $(\mathrm{p}=.049)$.

CONCLUSION: EBQI teams significantly improved outcomes for their depressed patient populations when and only when they implemented evidence-based programs. CTs were more likely than LTs to design and implement EB DIPs.

DOES AN INTERNIST-PSYCHIATRIS COLLABORATION MODEL FOR THE MANAGEMENT OF PSYCHIATRIC INPATIENTS IMPROVE CARE? A RANDOMIZED CONTROL TRIAL. A. Rubin ${ }^{1}$, B. Littenberg ${ }^{2}$, R. Ross ${ }^{2}$, S. Wehry ${ }^{1}$; ${ }^{1}$ University of Vermont, Burlington, VT; ${ }^{2}$ Fletcher Allen Health Care, Burlington, VT (Tracking ID \#73830)

BACKGROUND: People with mental illness are underserved in terms of preventive health services and chronic disease management. Several models have been proposed to address these disparities. These include training psychiatists in primary care and co-locating mental health and primary care services. We tested the effects of a third model, an internist-psychiatrist collaboration during an inpatient stay, on the outcomes and processes of care.

METHODS: We performed a randomized control study on an academic inpatient psychiatry unit. Patients in the intervention group met with the study internist who participated in their care by communicating with their primary care physicians, updating health maintenance and preventive endpoints, managing chronic and acute illensses during the stay, and attending work rounds. We looked at 139 adults admitted during a nine-month period. We measured functional status using the SF-36 and Basis-32, resource utilization using cost and length of stay, patient satisfaction using the PCAS, provider satisfaction using Likert scales for psychiatrists and referring physicians, and process of care using provision of such services as completion of data base, health maintenance preventive endpoints, and coordination of care. RESULTS: Patients in the intervention group showed large improvements in the processes of care (numerous measures with $\mathrm{p}<0.001$ ). Provider satisfaction improved overall, especially for the psychiatrists $(9.5$ vs.14.6: $\mathrm{p}=0.03$ ). Overall there was no impact on resource utilization. However, for medical comorbidity, the intervention group saw reduction of $\$ 4490$ in hospital $\operatorname{costs}(\mathrm{p}=0.027$ for the interaction between comorbidity and intervention) and in length of stay
( 8.1 fewer days; $\mathrm{p}=0.003$ for the interaction). There were no significant differences in function or patient satisfaction betwen the two groups.

CONCLUSION: Adding an internist to an inpatient psychiatric team is an effective and economical way of improving care in this traditionally underserved population. For the sickest patients, resource utilization improved dramatically.

EVERY SYSTEM IS PERFECTLY DESIGNED TO...: MISSED DIAGNOSIS OF HYPOTHYROIDISM UNCOVERED BY LINKING LAB AND PHARMACY DATA. G.D. Schiff ${ }^{1}$, S. Kim ${ }^{1}$, M.F. Wisniewski ${ }^{1}$, J. Bult ${ }^{1}$, L.A. Fogelfeld ${ }^{2}$, N. Krosnjar ${ }^{1}$; ${ }^{1}$ Cook County Hospital, Chicago, IL; ${ }^{2}$ University of Illinois at Chicago, Chicago, IL (Tracking ID \#75909)

BACKGROUND: Don Berwick has argued that "every system is perfectly designed to achieve the results it does" as a way of emphasizing both the systematic origins of many quality problems and the necessity to systematically change processes if significant and durable change is desired. As part of a larger AHRQ project examining diagnostic error, we studied the role of linking laboratory and pharmacy which are two often-disconnected disciplines and databases. This disconnection in information systems often results in overlooked improvement opportunities related to timely/effective care. Using linkages between lab and pharmacy data, we examined missed and delayed diagnosis of hypothyroidism by linking laboratory findings of hypothyroidism (elevated TSH) with a drug database to examine presence or absence of therapy (levothyroxine).

METHODS: We downloaded ASCII files of all TSH results (in- and outpatients) done during 2000 and 2001 in a public hospital system and electronically linked them to outpatient pharmacy prescriptions. All patients with TSH greater than 20 with no prescriptions were investigated with chart review and telephone follow-up to determine whether they were aware of and being treated for hypothyroidism, follow-up labs were evaluated and reordered, and treatment initiated where indicated.

RESULTS: A total of 22,077 TSH levels from 2000 and 24,524 from 2001 were drawn (17,467 and 19,293 unique patients, respectively). 1334 tests $(6.0 \%)$ were ? 20 ? $\mathrm{U} / \mathrm{ml}$ in 470 unique patients in 2000. In 2001, 744 tests were greater than 20 in 512 unique patients. Of the patients whose TSH was greater than 20,80 (17.0\%) in 2000 and $97(18.9 \%)$ in 2001 had no recorded prescriptions for levothyroxine; 17 out of 80 (3.6\%) in 2000 and 20 out of $97(3.9 \%)$ in 2001 were currently being treated for hyperthyroidism (excessive treatment of hyperthyroidism with antithyroid rx). 17 in 2000 and 34 in 2001 were receiving levothyroxine from outside pharmacies (not in our database), and 27 patients in both years were lost to follow-up. 12 $(2.6 \%)$ in 2000 and $11(2.1 \%)$ in 2001 of the patients with TSH > 20 were found to have missed hypothyroidism, 4 and 2 (year 2000 and 2001, respectively) others aware but failed follow-up. CONCLUSION: By linking lab and pharmacy databases, substantial \#s of patients with failed follow-up and treatment of abnormal TSH can be identified. Conservatively, 12 of $470(2.6 \%)$ in 2000 and 11 of $512(2.1 \%)$ in 2001 of all patients with TSH >20 definitely had missed diagnosis of hypothyroidism with another $27(5.7 \%)$ in 2000 and $27(5.3 \%)$ in 2001 lost to follow-up whose hypothyroidism also may be unrecognized. The results are virtually identical for each of two years studied retrospectively (except for use of outside pharmacy use which was being systematically promoted for Medicaid patients in 2001) demonstrating that we have a "perfectly designed system" to reproducibly generate this clinical diagnostic error. Analysis steps in the process where the errors occurred point to specific steps warranting system redesign.

QUALITY OF CARE FOR MEDICARE BENEFICIARIES ENROLLED IN FOR-PROFIT AND NOT-FOR-PROFIT HEALTH PLANS. E.C. Schneider ${ }^{1}$, A.M. Zaslavsky ${ }^{1}$, A.M. Epstein ${ }^{1} ;{ }^{1}$ Harvard University, Boston, MA (Tracking ID \#76936)

BACKGROUND: Prior research suggests that the quality of care in for-profit health plans is lower than that in not-for-profit health plans, however past studies have relied on voluntary health plan reporting of data. Past comparisons of quality of care could be biased if for-profit and not-for-profit health plans differed in their willingness to report.

METHODS: The Center for Medicare and Medicaid Services (CMS) mandates reporting of standardized data using HEDIS(TM) specifications. Merging these data with InterStudy data on health plan characteristics, we compared the quality of care of for-profit and not-for-profit health plans that enrolled Medicare beneficiaries aged 65 or older during 1997 . We studied four clinical services: breast cancer screening (BCS), diabetic eye examinations (DEE), betablocker medication after myocardial infarction (BBMI), and follow-up after hospitalization for mental illness (FHMI).

RESULTS: Among the 234 study health plans, $65 \%$ were for-profit. On all four of the HEDIS(TM) measures we studied, the quality of care was lower among for-profit health plans than among not-for-profit health plans $(67.4 \%$ vs. $74.4 \%$ for BCS, $44.0 \%$ vs. $57.8 \%$ for DEE, $63.7 \%$ vs. $75.5 \%$ for BBMI, and $41.9 \%$ vs. $61.6 \%$ for FHMI). After adjustment for sociodemographic case-mix and health plan characteristics (total enrollment, Medicaid enrollment, model type, age of health plan, and region) the quality of care remained lower in for-profit health plans than not-for-profit health plans, but these differences were not statistically significant for the BCS and BBMI measures.

CONCLUSION: Medicare beneficiaries enrolled in for-profit health plans receive lower quality of care than those enrolled in not-for-profit health plans. However part of the difference in quality of care may be explained by other features of for-profit health plans or the areas in which they operate.

MISSED MYOCARDIAL INFARCTION IN OUTPATIENTS WITH CHEST PAIN: THE USE OF THE FRAMINGHAM RISK SCORE. T.D. Sequist ${ }^{1}$, D.W. Bates ${ }^{1}$, E. Cook ${ }^{1}$, S. Lambert ${ }^{2}$,

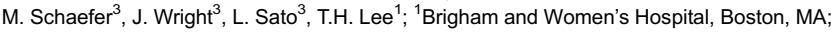
${ }^{2}$ Harvard Vabguard Medical Associates, Boston, MA; ${ }^{3}$ Risk Management Foundation of the Harvard Medical Institutions, Cambridge, MA (Tracking ID \#75821)

BACKGROUND: Missed diagnosis of myocardial infarction (MI) in the ambulatory setting is a major cause of patient morbidity and litigation. The Framingham Risk Score (FRS) is a well validated predictor of ten year coronary heart disease (CHD) risk. We evaluated the use 
of the FRS in patients with acute chest pain to identify those outpatients at high risk for missed MI.

METHODS: We performed a case-control study of patients with no previous history of CHD who presented with chest pain syndromes to Harvard affiliated primary care practices. 18 cases of missed diagnosis of MI were identified from malpractice claims files, with the diagnosis of MI occurring within 1 month of the office visit for chest pain. Control patients were matched on clinic location and date of encounter, with no diagnosis of $\mathrm{MI}$ in the month following an office visit for chest pain. Matching was performed using a 3:1 design (57 controls). Data included in the FRS was collected by physician chart review.

RESULTS: The Table shows that cases carried a substantially higher burden of Framingham cardiac risk factors compared to controls. Patients were significantly more likely to experience an $\mathrm{MI}$ in the presence of a FRS greater than $10 \%$, with a matched odds ratio of 7.8 (95 percent confidence interval 2.1 to 28.8 ).

CONCLUSION: Among patients with acute chest pain syndromes presenting to ambulatory practices, a Framingham risk score greater than $10 \%$ can identify those at increased risk of MI. This tool lends itself to use in the primary care setting, as the required information is typically readily available to outpatient clinicians.

\section{Table 1}

Age, years (median)

Male (\%)

Diabetic (\%)

Smoking (\%)

Total Cholesterol, mg/dL (median)

HDL Cholesterol, $\mathrm{mg} / \mathrm{dL}$ (median)

Systolic Blood Pressure, mmHg (median)

FRS $>10 \%(\%)$

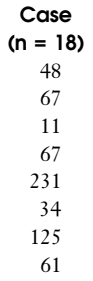

Control
$(\mathbf{n}=\mathbf{5 4})$
47
33
4
39
209
57
125
17

$P$ value

0.56

0.02

0.01

0.02

0.04

$<0.001$

0.74

$<0.001$

CLINICIAN ATTITUDES TOWARDS HEALTH MAINTENANCE AND DISEASE SPECIFIC PRACTICE GUIDELINES AND ELECTRONIC CLINICAL REMINDERS. T.D. Sequist ${ }^{1}$, T.K. Gandhi', E. Poon ${ }^{1}$, H. Murff', A. Karson ${ }^{3}$, D.W. Bates ${ }^{1}$; ${ }^{1}$ Brigham and Women's Hospital, Boston, MA; ${ }^{2}$ Vanderbilt University Medical Center, Nashville, TN; ${ }^{3}$ Massachusetts General Hospital, Boston, MA (Tracking ID \#76378)

BACKGROUND: Compliance with outpatient practice guidelines is low and clinical reminders have had variable success in improving adherence rates, particularly in chronic disease management. We evaluated primary care physician (PCP) attitudes towards compliance with guidelines, barriers to guideline adherence, and the use of electronic clinical reminders for routine health maintenance $(\mathrm{HM})$ and disease specific management recommendations.

METHODS: We surveyed 176 PCPs involved in a randomized controlled trial of electronic clinical reminders being conducted at 22 outpatient clinics in the Boston area. PCPs receive patient-specific clinical reminders within an outpatient electronic medical record. All physicians receive reminders for HM items (breast and cervical cancer screening, adult vaccinations), while the intervention group receives additional reminders for diabetes and coronary artery disease management. Surveys were mailed following exposure to the reminders for three months. The response rate was $63 \%(\mathrm{n}=111)$. Clinician responses were recorded using Likert scales and matched comparisons were performed using McNemar's test. RESULTS: Physicians were more likely to report at least moderate compliance with all routine HM guidelines $(86 \%)$ than with all disease specific management guidelines $(77 \%, p=0.01)$. $33 \%$ of physicians reported lack of knowledge of guideline recommendations as a barrier to adherence. In addition, $25 \%$ of physicians were aware of guideline recommendations, but forgot to apply them during an office visit. Among clinicians in the intervention group $(n=48)$, there was no difference in the number of physicians that found reminders regarding routine HM items useful $(56 \%)$ compared to reminders for diabetes management $(42 \%, \mathrm{p}=0.10)$ or coronary artery disease management $(46 \%, \mathrm{p}=0.46)$. Among all respondents, $29 \%$ reported acting on recommendations made by the electronic reminders during patient encounters. Overall, $74 \%$ of physicians felt that electronic clinical reminders significantly improved health care quality. CONCLUSION: PCPs report lower rates of compliance with disease specific management guidelines than for routine $\mathrm{HM}$ guidelines. Reminders can potentially remedy commonly sited barriers to guideline adherence, and disease management reminders are felt to be as useful as HM reminders. Patient-specific electronic clinical reminders frequently result in physician action and are felt to improve overall health care quality.

HOW WELL DO SMOKING CESSATION QUALITY MEASURES PREDICT CLINICAL OUTCOMES? S.E. Sherman ${ }^{1}$, E. Yano ${ }^{2}$, A.B. Lanto ${ }^{2}$, B.S. Mittman'; ; ${ }^{1}$ University of California, Los Angeles, Sepulveda, CA; ${ }^{2}$ VA Greater Los Angeles HSR\&D Center of Excellence, Sepulveda, CA (Tracking ID \#75327)

BACKGROUND: A wide range of quality of care measures for smoking cessation have been proposed or are currently in use. We assessed how well these structure and process measures correlate with clinical outcomes, such as smoking cessation.

METHODS: Our data are primarily from the Quality Improvement Trial for Smoking Cessation (QUITS), a recently completed trial of an organizational intervention to implement smoking cessation guidelines at 18 VA sites in the Southwestern US. We supplemented this with data from national VA databases. We measured the correlation between the 18 sites for structure quality of care measures (e.g., is there a system for screening), process quality of care measures (e.g., how frequently were patients counseled about smoking cessation), and clinical outcomes (quit attempts, smoking cessation). Our structure quality of care measure was the QUITS report card, a 25-item organizational checklist that assessed adherence to the system aspects of the smoking cessation guidelines. Our process quality of care measures included intensity scores for screening, counseling, referral, and treatment (from the QUITS administrative site survey); reported rate of smoking cessation counseling and medication prescription (QUITS patient survey); rate of smoking cessation clinic attendance (VA Outpatient Care File); and the actual rate of prescribing smoking cessation medications (VA Pharmacy Benefits Management database). Our outcome measures (from both the QUITS 12month and 18-month patient follow-up surveys) were the percent who had tried to quit recently and the percent who had actually quit smoking.

RESULTS: The percent who recently tried to quit at each site ranged from $27-59 \%$ at 12 months follow-up and from $27-52 \%$ at 18 months. The range of smoking cessation rates across the sites was $4-27 \%$ at 12 months and $3-20 \%$ at 18 months. Several of the potential quality measures correlated with each other to a moderate degree. However, none of them correlated with the rate of quit attempts or of actual cessation, either at 12-month or 18-month follow-up. CONCLUSION: Higher performance on a wide range of quality of care measures did not translate into more quitters or even more attempted quitters. Better quality of care measures may be needed for smoking cessation.

SMOKING CESSATION CARE RECEIVED BY VETERANS WITH CHRONIC OBSTRUCTIVE PULMONARY DISEASE (COPD). S.E. Sherman ${ }^{1}$, A.B. Lanto ${ }^{2}$, M. Nield ${ }^{2}$, E. Yano ${ }^{2} ;{ }^{1}$ University of California, Los Angeles, Sepulveda, CA; ${ }^{2}$ VA Greater Los Angeles HSR\&D Center of Excellence, Sepulveda, CA (Tracking ID \#76934)

BACKGROUND: Smoking is the main cause of COPD, and smoking cessation is the only effective intervention to slow its progression. We compared the cessation services received by smokers with COPD (COPD smokers) and smokers without COPD (non-COPD smokers) to determine whether COPD smokers received more intensive treatment.

METHODS: As part of a system-level guideline implementation study, we used computerassisted telephone interviewing to screen and enroll random samples of smokers within $18 \mathrm{VA}$ primary care clinics. Current smokers completed baseline and 12-month follow-up surveys (baseline $\mathrm{n}=1,941 ; 12$-months $\mathrm{n}=1,080$ ), composed of previously validated questions on smoking habits, history, and attitudes; health and functional status; and sociodemographics. Smokers reporting either emphysema or chronic bronchitis were classified as having COPD at baseline. We used multiple imputation using hot-deck techniques for missing values. We applied enrollment and attrition weights to all observations. We used $\dot{X}$ for discrete variables and ANOVA for continuous variables among COPD vs. non-COPD smokers and then controlled for confounding factors such as age and smoking history in multivariate regression. RESULTS: COPD smokers $(\mathrm{n}=522,27 \%)$ were older, more sedentary, more addicted to nicotine, and had worse physical and mental functioning (all $\mathrm{p}<0.001$ ) compared to nonCOPD smokers. COPD smokers were also more likely to report that smoking is harming their health $(91 \%$ vs. $84 \%, \mathrm{p}<0.001)$ and to report current problems caused by smoking $(86 \%$ vs. $68 \%, \mathrm{p}<0.001)$. COPD smokers also were more likely to report that within the last year that they had been advised to quit ( $74 \%$ vs. $64 \%$, OR $1.6,95 \%$ CI $1.3-2.0)$, prescribed nicotine patches $(32 \%$ vs. $22 \%$, OR $1.7,95 \%$ CI $1.4-2.1)$, or referred to a smoking cessation program (36\% vs. $26 \%$, OR $1.6,95 \%$ CI $1.3-1.9$ ). Receipt of these 3 smoking cessation services at 12 months follow-up was nearly identical to baseline levels. However, the rate of quitting smoking was the same for COPD smokers and non-COPD smokers (9.2\% vs. $9.0 \%)$. Most of the difference in smoking cessation services received by COPD smokers was for those in the precontemplation stage at baseline. Logistic regression showed that presence of COPD and level of nicotine addiction were independently associated with higher levels of each of these cessation services.

CONCLUSION: COPD smokers consistently received more smoking cessation services than non-COPD smokers, yet the quit rate was no higher. This suggests that new approaches may be required, perhaps because the preponderance of extra services received by COPD smokers were for those who were not interested in quitting.

PROCESSES AND OUTCOMES OF CARE IN ELDERLY PATIENTS WITH ACUTE BRONCHITIS. M.A. Steinman ${ }^{1}$, A. Sauaia ${ }^{2}$, J. Maselli ${ }^{3}$, P. Houck ${ }^{4}$, R. Gonzales ${ }^{3} ;{ }^{1}$ Univ. of California, San Francisco and San Francisco VA Medical Center, San Francisco, CA; ${ }^{2}$ Univ. of Colorado Health Sciences Center, Denver, CO; ${ }^{3}$ Univ. of California, San Francisco, San Francisco, CA; ${ }^{4}$ Centers for Medicare \& Medicaid Services, Seattle, WA (Tracking ID \#74145)

BACKGROUND: Acute bronchitis is predominantly a viral illness, the diagnosis of which depends largely on the exclusion of pneumonia based on clinical and radiographic characteristics. Among seniors, the unnecessary use of antibiotics for this condition is particularly problematic, due to elders' increased risk of subsequent antibiotic-resistant infections. We examined the frequency, determinants, and outcomes of antibiotic use in elderly patients with acute bronchitis.

METHODS: Medicare administrative data were used to identify a stratified random sample of seniors with acute bronchitis from outpatient practices in the Denver metropolitan area. Medical record review was performed to confirm the diagnosis and assess processes of care for these patients; seniors with interstitial or chronic obstructive lung disease were excluded. Data on subsequent health care utilization were obtained from Medicare billing records. All analyses were done using generalized estimating equations to account for the clustered nature of the data. RESULTS: Of 198 patients with acute bronchitis, 61\% were female, the mean age was 76 (SD 8.6 ), and $53 \%$ had at least one comorbid condition. Chest X-rays were ordered in $10 \%$ of patients, and vital signs (important clinical predictors of pneumonia) were often not recorded: temperature was missing from $34 \%$ of charts and pulse from $50 \%$ of charts. When recorded, significant vital sign abnormalities were uncommon, with $8 \%$ having temperature or pulse $>100$. Antibiotics were prescribed to $83 \%$ of patients. Treatment with antibiotics was more common among men than women $(92 \%$ vs. $78 \%, \mathrm{P}<.01)$, but was not associated with vital sign measurement, vital sign results, orders for chest X-ray, patient age, duration of illness, or the presence of comorbidities. In addition, antibiotic prescribing was not associated with the rate of return office visits within 2 weeks of the index visit, even after stratifying patients into risk groups based on vital sign abnormalities and comorbidities. These return visits were made by $13 \%$ of patients during this period. 
CONCLUSION: Vital signs and chest X-rays are frequently not performed in seniors with acute bronchitis. However, the vast majority of these patients receive antibiotics, independent of patient characteristics or the type of workup received. In addition to patient education campaigns to curtail demand for antibiotics, reducing inappropriate antibiotic use in seniors will likely depend on improving the workup of these patients and encouraging clinicians to act appropriately on the results.

ARE CLINICIANS AWARE OF THEIR ADHERENCE TO HYPERTENSION GUIDELINES? M.A. Steinman ${ }^{1}$, M.A. Fischer ${ }^{2}$, M. Shlipak ${ }^{1}$, H.B. Bosworth ${ }^{3}$, E.Z. Oddone ${ }^{3}$, M.K. Goldstein $^{2}$; ${ }^{1}$ UCSF and San Francisco VAMC, San Francisco, CA; ${ }^{2}$ Palo Alto VAMC and Stanford University, Palo Alto, CA; ${ }^{3}$ Duke University and Durham VAMC, Durham, NC (Tracking ID \#74148)

BACKGROUND: Knowledge of current practices is important for improving future performance, yet little is known about how well physicians are aware of their own adherence to clinical guidelines. We compared clinicians' beliefs about their adherence to hypertension guidelines with data on their actual guideline performance.

METHODS: We surveyed 223 primary care clinicians at 3 VA medical centers, asking providers to assess their own adherence to hypertension guidelines. Using a validated algorithm, we extracted data from VA clinical databases on guideline-concordant medication use and blood pressure control for patients with hypertension assigned to these providers. Data were collected on patients with hypertension accompanied by diabetes, coronary disease, or neither comorbidity.

RESULTS: 137 clinicians completed the survey; clinical data was available for 86 of these respondents. The mean panel size was 117 patients, mean patient age was 65 , and patients were on an average of 1.6 anti-hypertensive medications. Overall, clinicians overestimated the proportion of their patients on guideline-concordant medications ( $75 \%$ perceived vs. $66 \%$ actual ), and with blood pressure $<140 / 90$ on their last visit ( $66 \%$ perceived vs. $43 \%$ actual; $\mathrm{P}<$ 001 for both comparisons). Among individual clinicians, we found poor correlation between perceived and actual guideline adherence $(r=0.19$ for medications and 0.14 for blood pressure control; $\mathrm{P} \geq .10$ for both). For example, clinicians in the lowest and highest quartiles of actual blood pressure control perceived nearly identical levels of adherence to this guideline $(68 \%$ vs. $69 \%$; $>.20$ for difference). Over one-third of clinicians overestimated by $>15 \%$ the proportion of their patients on guideline-concordant medications; a similar number overestimated by $>30 \%$ the proportion of their patients meeting blood pressure targets. On multivariable analysis, we found no association between provider demographics, practice characteristics, guideline familiarity, or attitudes toward guidelines and the likelihood of overestimating one's own adherence to either guideline measure.

CONCLUSION: Clinicians may overestimate their adherence to hypertension guidelines, particularly the number of their patients meeting blood pressure targets. This limited awareness may represent a barrier to successful implementation of guidelines, and could be addressed through the use of patient profiles and point-of-service feedback to clinicians.

THE SAFETY OF PATIENTS ISOLATED FOR INFECTION CONTROL. H.T. Stelfox ${ }^{1}$, D.W. Bates ${ }^{2}$, D.A. Redelmeier ${ }^{3} ;{ }^{1}$ Harvard University, Brookline, MA; ${ }^{2}$ Brigham and Women's Hospital, Watertown, MA; ${ }^{3}$ University of Toronto, Toronto, Ontario (Tracking ID \#74941)

BACKGROUND: Hospital infection control policies that employ patient isolation prevent the nosocomial transmission of infectious diseases, but may inadvertently lead to patient neglect. We tested whether isolation strategies were associated with the quality of medical care received during hospitalization.

METHODS: We identified consecutive adults admitted to two large North American teaching hospitals between January 1, 1999 and July 1, 2002 who were isolated for methicillin resistant Staphylococcus aureus (MRSA). Two control patients were selected for each isolated patient by matching on hospital bed (Canadian center) and admission diagnosis (American center). Quality of care measures encompassed three domains of care: processes, outcomes, and patient satisfaction. Adjustments for demographic (age, gender, primary language, institutional status, household income), clinical (individual comorbidities, Charlson Index score, APACHEII score, DNR status, admitting diagnosis) and hospital (mode of arrival, admitting service, ward) characteristics were conducted using multivariable regression analyses.

RESULTS: We found that isolated patients $(\mathrm{n}=150)$ compared to control patients $(\mathrm{n}=300)$ were more likely to have their vital signs not recorded as ordered ( $51 \%$ vs. $31 \%, \mathrm{p}<0.001)$ and more likely to have no daily physician progress note recorded ( $25 \%$ vs. $13 \%, \mathrm{p}<0.001)$. Isolated patients were more likely than control patients to experience an adverse event during their hospitalization (22 per 1000 days vs. 14 per 1000 days, $\mathrm{p}=0.007)$. This difference reflected preventable (17 per 1000 days vs. 3 per 1000 days, $\mathrm{p}<0.001)$ as opposed to non-preventable (11 per 1000 days vs. 11 per 1000 days, $p>0.2)$ adverse events. No differences in hospital mortality were observed for the two groups $(17 \%$ vs. $10 \%, \mathrm{p}=0.180)$. Isolated patients were more likely to formally complain to the hospital about their care than control patients $(10 \%$ vs. $1 \%, p=0.003)$. CONCLUSION: Patients isolated for infection control appear to have less care documented, experience more preventable adverse events and express greater dissatisfaction with their treatment than control patients.

ERRORS IN OUTPATIENT AMIODARONE MONITORING. H.T. Stelfox ${ }^{1}$, S.B. Ahmed ${ }^{2}$, J. Fiskio ${ }^{3}$, D.W. Bates ${ }^{4} ;{ }^{1}$ Harvard University, Brookline, MA; ${ }^{2}$ Brigham and Women's Hospital, Brookline, MA; ${ }^{3}$ Partners Information Systems, Boston, MA; ${ }^{4}$ Brigham and Women's Hospital, Watertown, MA (Tracking ID \#74970)

BACKGROUND: Medication monitoring may prevent or minimize the consequences of adverse drug events (ADEs). We sought to develop an explicit model of medication monitoring and to evaluate monitoring errors and ADEs in patients taking amiodarone.

METHODS: A retrospective chart review of 99 outpatients receiving amiodarone therapy between January 1, 2000 and January 1, 2001 at a large tertiary care hospital. Explicit monitoring criteria derived from the literature (MEDLINE 1966 to 2000) and through expert opinion were used to evaluate patients' medical records to assess adherence. Adverse drug events were identified using structured implicit reviews. The main outcome measures were the proportion of patients satisfying minimum monitoring criteria, experiencing specific monitoring errors and having amiodarone-related ADEs.

RESULTS: Overall, only $9 \%$ (95\% confidence interval [95\% CI] 3\%-15\%) of the patients prescribed amiodarone received the minimal recommended monitoring. Errors were identified at all stages of the monitoring model. Fifty-two percent $(95 \%$ CI $42 \%-62 \%)$ of the patients received minimum baseline evaluations, $22 \%$ (95\% CI 14\%-31\%) ongoing surveillance, $75 \%$ $(95 \% \mathrm{CI} 61 \%-89 \%)$ appropriate responses to abnormal surveillance results and $71 \%(95 \% \mathrm{CI}$ $62 \%-80 \%)$ timely follow-up visits. Eight percent $(95 \%$ CI $3 \%-13 \%)$ of the patients experienced an amiodarone-related $\mathrm{ADE}$, of which $33 \%$ were judged to be attributable to monitoring errors. Interrater agreements for monitoring processes $(k=0.83)$ and ADEs $(\mathrm{k}=0.67)$ were good.

CONCLUSION: Only $9 \%$ of outpatients taking amiodarone at one tertiary care hospital received recommended monitoring during 1 year of follow-up. Amiodarone has complex monitoring requirements and it may be difficult for all criteria to be simultaneously satisfied. Some amiodarone-related ADEs are associated with monitoring errors.

AN EVALUATION OF THE ADEQUACY OF OUTPATIENT MONITORING OF THYROID REPLACEMENT THERAPY. H.T. Stelfox ${ }^{1}$, S.B. Ahmed ${ }^{2}$, J. Fiskio ${ }^{3}$, D.W. Bates ${ }^{4} ;{ }^{1}$ Harvard University, Brookline, MA; ${ }^{2}$ Brigham and Women's Hospital, Brookline, MA; ${ }^{3}$ Partners Information Systems, Boston, MA; ${ }^{4}$ Brigham and Women's Hospital, Watertown, MA (Tracking ID \#74972)

BACKGROUND: Hypothyroid patients managed with excessive or insufficient thyroid replacement therapy are often difficult to clinically recognize. Monitoring may prevent or minimize the consequences of adverse drug events (ADEs). We sought to develop an explicit model of medication monitoring and to evaluate monitoring processes and ADEs in patients taking levothyroxine.

METHODS: A retrospective chart review of 400 outpatients receiving levothyroxine therapy between January 1, 2000 and January 1, 2001 at a large tertiary care hospital. Explicit monitoring criteria derived from the literature (MEDLINE 1966 to 2000) and through expert opinion were used to evaluate patients' medical records to assess adherence. Adverse drug events were identified using structured implicit reviews. We measured the proportion of patients satisfying minimum monitoring criteria and experiencing levothyroxine-related ADEs. RESULTS: Overall, only $56 \%$ (95\% confidence interval [ $95 \%$ CI] $51 \%-62 \%$ ) of the patients prescribed levothyroxine received the minimal recommended monitoring. Errors were identified at all stages of the monitoring model. Patients who received the recommended monitoring had fewer levothyroxine-related ADEs than those who did not $(1 \%$ vs. $6 \%$, $\mathrm{p}=0.013$ ). Minority status (Caucasians $2 \%$ vs. African Americans $4 \%$ vs. Hispanics $14 \%$, $\mathrm{p}=0.023$ ) and primary language (English $3 \%$ vs. Non-English $20 \%, \mathrm{p}=0.002$ ) were the patient characteristics also associated with levothyroxine-related ADEs. Interrater agreement for monitoring processes $(\mathrm{k}=0.89)$ and ADEs $(\mathrm{k}=0.75)$ were good.

CONCLUSION: Only half of outpatients taking levothyroxine at one tertiary care hospital received the recommended monitoring during 1 year of follow-up. Levothyroxine-related ADEs were more frequent in patients with lower-quality monitoring and in minorities and non-English speakers.

MEASURES OF THE QUALITY OF INTERPERSONAL PROCESSES OF CARE: PRELIMINARY PSYCHOMETRIC RESULTS IN LATINO, AFRICAN AMERICAN, AND WHITE GENERAL MEDICINE PATIENTS. A.L. Stewart ${ }^{1}$, A.M. Nápoles-Springer ${ }^{1}$, S. Gregorich ${ }^{1}$, J. Santoyo ${ }^{1}$; ${ }^{1}$ University of California, San Francisco, San Francisco, CA (Tracking ID \#74153)

BACKGROUND: Interpersonal processes of care (IPC) between physicians and patients could potentially explain racial/ethnic disparities in health. Poorer IPC could result in lower levels of knowledge, adherence, and satisfaction, leading to poorer health. To explore these hypotheses, measures of IPC are needed that are valid across groups.

METHODS: Based on an earlier instrument and qualitative studies, we developed a revised patient-report instrument measuring 3 broad domains of physicians' IPC: communication, decision making, and interpersonal style. The instrument was administered to patients aged 18 and older from a large adult general medicine continuity practice who had at least 2 visits during the prior year. We sampled 4 groups: English-speaking Latinos (LE), Spanish-speaking Latinos (LS), African Americans (AA), and Whites (WH). Respondents reported the frequency with which their primary care physicians performed various aspects of IPC over the 6 months prior to the interview. Multitrait scaling was used to assess the hypothesized measurement structure and to assure that resulting scales met psychometric criteria in each group; we assessed item convergence within scales, item discrimination across scales, and internalconsistency reliability.

RESULTS: We obtained 1,664 surveys (428 LE, $383 \mathrm{LS}, 435 \mathrm{AA}$, and $418 \mathrm{WH}$ ); age ranged from $18-99(M=51, S D=18) ; 71 \%$ were women and $35 \%$ had $\leq$ HS education. The preliminary 51-item instrument contained 10 scales that demonstrated adequate psychometric properties in all groups: 5 communication scales (general clarity; elicitation/responsiveness to patient concerns; explanations of condition, processes of care, and self-care), 1 shared decisionmaking scale, and 4 interpersonal style scales (friendliness/respect, perceived discrimination, emotional support, and empowerment). Reliabilities were excellent across groups for 9 scales $(.79-.91)$ but lower for general clarity $(.63-.69)$. Differences in quality of IPC based on mean scores (range $0-100$, higher $=$ better quality), adjusted for age and gender, were found across scales. In all groups, the highest quality was reported for discrimination ( $M$ ranged from 92-95 across groups, indicating very low levels of discrimination) and friendliness/respect (M ranged from 84-89); the lowest quality was observed for shared decision-making (M 49-55) and explanations about self-care (M 45-59). We are also using confirmatory factor analyses (CFA) methods to examine the psychometric invariance of the scales across groups; final scales will be based on the convergence of results from multitrait scaling and CFA methods.

CONCLUSION: Few measures of reports of interpersonal processes of care are available that meet psychometric criteria across diverse groups, due to inherent challenges in this research. 
The final instrument should be useful for examining how IPC differentially affects intermediate and ultimate outcomes of care across these racial/ethnic groups.

IMPROVING PATIENT CARE AND OUTCOMES IN HEART FAILURE: A CONTROLLED TRIAL OF COMPUTER-BASED CARE SUGGESTIONS ENHANCED BY PATIENTS SYMPTOMS AND THEIR SEVERITY. U. Subramanian ${ }^{1}$, S.D. Fihn ${ }^{2}$, M. Weinberger ${ }^{3}$, L. Plue ${ }^{4}$, F. Smith ${ }^{4}$, E. Udris ${ }^{2}$, M. McDonnell ${ }^{2}$, M. Temkit ${ }^{4}$, G. Eckert ${ }^{4}$, A. Zhou ${ }^{4}$, L. Chen ${ }^{5}$ W.M. Tierney ${ }^{6}$; ${ }^{1}$ Roudebush VAMC, Indiana University, Indianapolis, IN; ${ }^{2}$ University of Washington, Seattle, WA; ${ }^{3}$ University of North Carolina at Chapel Hill, Chapel Hill, NC; ${ }^{4}$ Indiana University, Indianapolis, IN; ${ }^{5}$ University of Rochester, Rochester, NY; ${ }^{6}$ Indiana University Purdue University Indianapolis, Indianapolis, IN (Tracking ID \#75104)

BACKGROUND: Heart failure (HF) is common, morbid and costly. Yet, physicians' adherence to treatment guidelines is suboptimal. Objective: To assess whether providing primary care physicians with guideline-based care suggestions based, in part on patients' symptoms would improve outcomes of patients with $\mathrm{HF}$.

METHODS: Randomized controlled trial in two university-affiliated VA primary care clinics. Patients were eligible if they had a diagnosis of $\mathrm{HF}$ and objective evidence of left ventricular systolic dysfunction. Intervention: Enhanced care involving guideline-based suggestions using data from electronic medical records (EMR) and recent symptoms, severity, and changes. Control care involved suggestions based on EMR data alone. Outcomes: Adherence to $\mathrm{HF}$ guidelines; New York Heart Association (NYHA) class; generic (SF-36) and cardiovascularspecific (McMaster-CHF) health status; satisfaction with care; hospital days; and outpatient visits. We report results at 12 months

RESULTS: Enhanced care $(\mathrm{N}=360)$ and control patients $(\mathrm{N}=386)$ were comparable at baseline. Significant $(\mathrm{p}<0.0001)$ within-group improvement was observed for adherence to guidelines, McMaster CHF, and NHYA class. $52 \%$ of enhanced care patients and $43 \%$ of control care group improved in their NYHA class. However, there were no significant differences between groups on adherence to CHF guidelines ( $34 \%$ vs. $27 \%, \mathrm{p}=0.32$ ), NYHA class ( $\mathrm{p}=0.3)$, any SF- 36 or McMaster-CHF scale $(\mathrm{p}>0.1)$, hospital days ( 0.82 vs. $0.83 /$ patient, $\mathrm{p}=0.8)$ or outpatient visits $(9.15$ vs. $9.5 /$ patient, $\mathrm{p}=0.4)$. Enhanced care patients were more satisfied with their physicians $(\mathrm{p}=.01)$ and primary care $(\mathrm{p}=0.047)$.

CONCLUSION: Guideline-based suggestions using both EMR and symptom data did not improve patients' outcomes beyond suggestions based on EMR data alone. Primary care practices with EMRs should use available clinical data to enhance the care and outcomes of patients with $\mathrm{CHF}$

RISING RATES OF OPIOID PURCHASES NOT GOING TO HOSPITALIZED PATIENTS DURING THEIR FINAL WEEK OF LIFE. S.W. Tolle ${ }^{1}$, S.E. Hickman ${ }^{1}$, V.P. Tilden ${ }^{1}$ J.S. Bubalo ${ }^{1}$, E.K. Fromme ${ }^{1} ;{ }^{1}$ Oregon Health \& Science University, Portland, OR (Tracking ID \#76250)

BACKGROUND: Substantial resources have been spent to improve pain control for dying patients and increased opioid administration has been presumed. The Automation of Reports and Consolidated Orders System (ARCOS) reports the number of grams of opioids purchased per 100,000 population quarterly. Oregon has been a consistent leader in per capita purchasing for morphine for the past 10 years. Health policy experts, extrapolating from World Health Organization methods, have suggested these data are indicative of the quality of end of life care in Oregon. We undertook a study to determine whether trends in opioid prescription at the state and national levels reflect opioid use for inpatients during the final week of life. METHODS: We reviewed the charts of the 877 inpatients who died at Oregon Health \& Science University from natural causes between 1-1-97 and 12-31-99, recording all opioid medications administered during the last days (maximum 7 days). Opioid medications were converted to oral morphine equivalents, totaled per patient, and then converted to grams of morphine per 100,000 dying persons. ARCOS data in grams per 100,000 population were also converted into oral morphine equivalencies for comparison purposes. Regressions were performed using the OHSU inpatient, Oregon ARCOS, and US ARCOS data with time period ( $\mathrm{n}=12$ quarter years) as the independent variable and average grams $/ 100,000$ population as the dependent variable. Comparisons were performed for morphine alone and for all other opioids in oral morphine equivalencies.

RESULTS: OHSU inpatient morphine use did not increase significantly for dying patients from 1997-1999 (beta = .139, $\mathrm{p}=.666$ ). However, overall morphine purchasing for both Oregon and US populations increased significantly (Beta $=.837, \mathrm{p}=.011$ and Beta $=.885, \mathrm{p}<.001$, respectively). Pair wise $\mathrm{R}$ to $\mathrm{Z}$ transformations showed no significant difference between linear trends for Oregon and US populations $(\mathrm{z}=.41, \mathrm{p}=.35)$, but both were significantly different from the OHSU inpatient sample $(\mathrm{z}=-2.27, \mathrm{p}=.011$ and $\mathrm{z}=-2.68, \mathrm{p}=.003$, respectively). The findings were similar for all other opioids in oral morphine equivalencies.

CONCLUSION: There was no significant change in the amount of morphine or other opioids administered to dying patients over a 3 year period at a large academic medical center, despite Oregon and national ARCOS data showing significant increases in purchases of morphine and other opioids. These findings suggest that ARCOS data does not by itself provide information about opioid use or pain management for specific populations and that the actively dying may not be receiving the same increases in opioids as other patient subgroups.

TREATMENT ADEQUACY WITH SELECTED ANTIDEPRESSANTS BY GENERALIST PHYSICIANS. G.J. Wan ${ }^{1}$, K. Yu-Isenberg ${ }^{2}$, C.L. Fontes ${ }^{2}$; ${ }^{1}$ Wyeth Research, St. Davids, PA; ${ }^{2}$ Prescription Solutions, Costa Mesa, CA (Tracking ID \#76593)

BACKGROUND: Current goals of antidepressant management in primary care include treatment adequacy. The objective of the study was to examine treatment adequacy rates with venlafaxine extended-release (VENXR) or fluoxetine by generalist physicians.

METHODS: Retrospective analysis was performed using Pacificare Health Systems and Prescription Solutions data. Treatment adequacy was defined using the Health Plan Employer Data and Information Set (HEDIS) Antidepressant Medication Management measures as continuous therapy for 84 or 180 days at a specified dose (75-150 mg/day for VENXR; $20 \mathrm{mg}$ / day for fluoxetine). Pharmacy claims were obtained for 90 days prior and 270 days after the index prescription for either VENXR or fluoxetine during the index period $(1 / 1 / 00-2 / 28 / 01)$. Generalist physicians (family practice, general practice or internal medicine specialty) prescribed the first prescription of the index antidepressant. The cohort included patients newly starting with either VENXR or fluoxetine and remaining on the same medication for 84 or 180 continuous days.

RESULTS: VENXR $(n=845)$ had an adequacy rate of $85 \%$ versus $62 \%$ for fluoxetine $(\mathrm{n}=1,964)$ for 84 continuous days $(\mathrm{P}<0.0001)$. VENXR $(\mathrm{n}=537)$ had an adequacy rate of $86 \%$ versus $58 \%$ for fluoxetine $(\mathrm{n}=1,328)$ for 180 continuous days $(\mathrm{P}<0.0001)$. The adjusted odds ratios $(\mathrm{OR})$ of achieving treatment adequacy with VENXR versus fluoxetine were 3.51 $(95 \% \mathrm{CI}=2.84-4.34)$ for 84 continuous days and $4.60(95 \% \mathrm{CI}=3.51-6.02)$ for 180 continuous days.

CONCLUSION: Patients prescribed VENXR achieved higher treatment adequacy rates versus fluoxetine. High treatment adequacy rates may lead to improved therapeutic outcomes in these patients treated with antidepressants by generalist physicians.

DIFFERENCES IN THE REPORTING OF CARE-RELATED PATIENT INJURIES TO EXISTING REPORTING SYSTEMS. L.K. Williams ${ }^{1}$, M. Pladevall ${ }^{1}$, A.M. Fendrick ${ }^{2}$, J. ElstonLafata $^{1}$, L. McMahon ${ }^{2} ;{ }^{1}$ Henry Ford Health System, Detroit, MI; ${ }^{2}$ University of Michigan, Ann Arbor, MI (Tracking ID \#76343)

BACKGROUND: Care which results in the injury of a patient can signal a breakdown in an institution's safety systems. Although reporting these events may result in steps to prevent their future occurrence, this requires healthcare provider participation. Currently, it is unknown which type of system encourages the most reporting. Therefore, the purpose of this study is to compare the number and type of care-related injuries reported to existing systems.

METHODS: We surveyed all states with laws requiring hospitals to report care-related patien injuries. Officials in these states were asked to provide information about the number and types of care-related events reported to them in 1999. In addition, the Joint Commission on Accreditation of Healthcare Organizations (JCAHO) provided information about events reported to it in 1999 .

RESULTS: Nine states used definitions similar to those used by the JCAHO for care-related patient injuries. In all categories examined, the number of reports submitted by accredited hospitals to states equaled or exceeded the number reported to the JCAHO.

CONCLUSION: State reporting systems identified a greater number of care-related injuries when compared with the JCAHO system. These differences may have important safety implications in regard to learning about and preventing care-related injuries.

THE RELATIONSHIP OF CARE SITE HIV SPECIALIZATION TO VARIATIONS IN RATES OF ADOPTION OF HAART THERAPY. I.B. Wilson ${ }^{1}$, B.E. Landon ${ }^{2}$, L. Ding ${ }^{2}$, A.M. Zaslavsky ${ }^{2}$, M.F. Shapiro ${ }^{3}$, S.A. Bozzette ${ }^{4}$, P.D. Cleary ${ }^{2} ;{ }^{1} \mathrm{New}$ England Medical Center, Boston, MA; ${ }^{2}$ Harvard University, Boston, MA; ${ }^{3}$ University of California, Los Angeles, Los Angeles, CA; ${ }^{4}$ University of California, San Diego, San Diego, CA (Tracking ID \#75475)

BACKGROUND: Little is known about the relationships between the characteristics of sites where HIV care is provided in the US and rates of use of highly active antiretroviral therapy (HAART)

METHODS: We conducted a cross-sectional analysis of data from the HIV Costs and Service Utilization Study (HCSUS), a nationally representative sample of patients with HIV. Data were from patient, site (response rate (RR) 70\%), and physician surveys (RR 75\%). The analytic dataset included $80.3 \%$ (2300/2864). of the baseline HCSUS sample. The dependent variable was use of HAART by December of 1996. Site characteristics of sites including HIV specialization; nurse, case management and MD staffing levels; total visit volumes (HIV and non-HIV); coverage arrangements; support services (e.g., mental health); financial incentives; quality and utilization management strategies; and demographic characteristics of the zip code area in which the site was located. We estimated hierarchical logistic regression models that nested patients within sites. Data were weighted for sampling and non-response. All analyses adjusted for patient characteristics.

RESULTS: $78 \%$ of patients nationally received care at a site specializing in HIV care. Compared with non-HIV sites, HIV sites had smaller patient volumes, more infectious diseases specialists, and tended to care for patients with more advanced HIV disease (for all, $\mathrm{p}<0.01$ ). Over $90 \%$ of patients were cared for by experts in HIV care, either infectious diseases specialists $(46 \%)$ or general medicine experts $(45 \%)$. Rates of HAART use by site ranged from 0.02-0.79 (mean 0.33). In multivariable models, HIV site (OR 3.6, $\mathrm{p}<0.001$ ), total patient volume of $>20,000$ visits a year (OR 2.1, p $<0.01$ ), and educational level of the zip code in which the site was located (OR 1.2 for each $10 \%$ increase in percent college educated) were al associated with higher rates of HAART use. These effects persisted even after adjustment for physician HIV expertise. Model building was repeated without the HIV site expertise variable, and no additional variables were significant.

CONCLUSION: Most of the patients in this country with HIV receive care at sites that specialize in HIV care, and from providers that are HIV experts. More HIV expertise and greater patient volumes, but none of the many structural characteristics of sites that we assessed, were associated with higher rates of HAART use.

A COMPLAINT IS A GIFT: QUALITATIVE STUDY OF UNSOLICITED PATIENT COMPLAINTS FOR USE IN MEDICAL EDUCATION. J.L. Wofford ${ }^{1}$, M.M. Wofford ${ }^{1}$, S.B. Kendrick ${ }^{1}$, J. Bothra ${ }^{2}$, A. Smith ${ }^{1}$, P.R. Lichstein ${ }^{1} ;{ }^{1}$ Wake Forest University, WinstonSalem, NC; ${ }^{2}$ Empire State College, Saratoga Springs, NY (Tracking ID \#74544)

BACKGROUND: Health care institutions are required by accrediting institutions to routinely collect and address formal patient complaints. Despite the availability of this form of customer feedback, there are no published efforts exploring such data for the purposes of improving 
communication skills in medical education. We sought to determine the usefulness of patient complaints by establishing meaningful categories and exploring their epidemiology.

METHODS: Using a register of formal, unsolicited patient complaints collected routinely at a single academic medical center, we categorized complaints using qualitative research strategies. After eliminating complaints unrelated to physician behavior, we analyzed patient complaints from one month $(\mathrm{n}=60)$ to derive complaint categories and then validated these categories using a second month of complaints $(\mathrm{n}=122)$. After establishing seven complaint categories, we then examined all complaints over a one year period.

RESULTS: Patient complaints unrelated to physician behavior (i.e., billing, food service, etc.) $(\mathrm{n}=1342)$ and complaints with inadequate detail $(\mathrm{n}=182)$ were excluded, leaving a total of 222 complaints for further analysis (222/1342,16.5\%). Complaints were most commonly lodged by a patient (111), followed by a patient's spouse (31), child (52), parent (50), relative/friend (15), and health care professional (2). The most commonly identified category was disrespect $(36.0 \%, 80 / 222)$, followed by disagreement about expectations of care $(23.4 \%)$, inadequate information $(20.4 \%)$, distrust $(18.0 \%)$, perceived unavailability $(14.9 \%)$, interdisciplinary miscommunication $(4.1 \%)$, and misinformation $(4.1 \%)$. Multiple categories were identified in $20.3 \%(42 / 222)$ of complaints. Examples from each complaint category provide adequate detail to develop instructional modules.

CONCLUSION: Seven distinct categories of unsolicited patient complaints were identified through this qualitative research process. Disrespect was the most commonly identified complaint category at this institution. These complaint categories should be useful in developing curricula related to professionalism and communication skills.

COMPARISON OF MEDICATION USE IN CORONARY HEART DISEASE PATIENTS WITH AND WITHOUT DIABETES. L.D. Woodard ${ }^{1}$, N.R. Kressin ${ }^{2}$, L.A. Petersen ${ }^{1} ;{ }^{1}$ Houston Center for Quality of Care and Utilization Studies, Houston, TX; ${ }^{2}$ Boston University, Bedford, MA (Tracking ID \#74894)

BACKGROUND: Coronary heart disease (CHD) is the leading cause of death among patients with diabetes. Secondary prevention in patients with diabetes confers benefits comparable to or greater than those seen in patients without diabetes. However, diabetic patients are often less likely to receive such therapies. Our goal was to compare the use of medications for secondary prevention among diabetic and non-diabetic patients treated within the Veterans Health Administration.

METHODS: 1045 veterans with CHD established by positive nuclear imaging study were enrolled at five Veterans Administration Medical Centers. Ideal candidates for beta-blockers, angiotensin converting enzyme (ACE) inhibitors, lipid-lowering agents, and aspirin were identified using recommendations from the American College of Cardiology (ACC)/American Heart Association (AHA) practice guidelines for the management of patients with unstable angina. Differences between diabetic and non-diabetic patients were assessed using chi-square and t-tests.

RESULTS: Of the 1045 patients, 335 (32.3\%) had diabetes. There were no differences between diabetic and non-diabetic patients in mean age (63.5 years [ \pm 9.3$]$ vs. 62.4 years $[ \pm 10.4] ; \mathrm{P}=0.08)$, mean education (11.9 years $[ \pm 2.7]$ vs. 12.1 years $[ \pm 2.8] ; \mathrm{P}=0.33$ ), percentage of AfricanAmericans $(24.8 \%$ vs. $21.4 \% ; \mathrm{P}=0.22)$, percentage of unmarried subjects $(38.6 \%$ vs. $42.4 \%$; $\mathrm{P}=0.24)$, or income $<\$ 20,000(64.5 \%$ vs. $66.9 \% ; \mathrm{P}=0.47)$. Diabetic patients were more likely than non-diabetic patients to have a diagnosis of hypertension $(86.3 \%$ vs. $74.5 \% ; \mathrm{P}<0.0001)$, hypercholesterolemia $(70.8 \%$ vs. $58.8 \% ; \mathrm{P}=0.0002)$, peripheral arterial disease $(22.5 \%$ vs. $14.9 \% ; \mathrm{P}=0.003)$, and renal disease $(13.3 \%$ vs. $5.6 \% ; \mathrm{P}<0.0001)$. Among ideal candidates for secondary prevention therapies, diabetic patients were more likely than non-diabetic patients to receive aspirin $(80.0 \%$ vs. $72.0 \% ; \mathrm{P}=0.02)$ and $\mathrm{ACE}$ inhibitors $(73.0 \%$ vs. $42.6 \% ; \mathrm{P}<0.0001)$. There were no significant differences in use of beta-blockers $(59.3 \%$ vs. $51.8 \% ; \mathrm{P}=0.12)$ or lipid-lowering agents $(99.5 \%$ vs. $97.9 \% ; \mathrm{P}=0.13)$. Among patients who were ideal candidates for treatment with multiple medications, diabetic patients were significantly more likely than non-diabetic patients to receive two $(85.9 \%$ vs. $62.4 \%$; $\mathrm{P}<0.0001)$, three $(60.2 \%$ vs. $47.8 \%$; $\mathrm{P}=0.02)$, or four $(47.6 \%$ vs. $22.7 \%$; $\mathrm{P}=0.0007)$ medications simultaneously.

CONCLUSION: Diabetic patients with CHD were equally or more likely than non-diabetic patients to receive treatment with beta-blockers, lipid-lowering agents, ACE inhibitors, and aspirin. Although diabetic patients were equally or more likely to be treated with these single agents, overall rates of treatment with beta-blockers and ACE inhibitors could be improved in both groups to ensure maximal benefit in secondary prevention. 